\title{
Performance of the ATLAS Hadronic End-Cap Calorimeter in Beam Tests
}

ATLAS Liquid Argon HEC Collaboration

B. Dowler, J. Pinfold, J. Soukup, M. Vincter

University of Alberta, Edmonton, Canada

A. Cheplakov, V. Datskov, A. Fedorov, N. Javadov ${ }^{1}$, V. Kalinnikov, S. Kakurin, M. Kazarinov, V. Kukhtin, E. Ladygin, A. Lazarev, A. Neganov, I. Pisarev, E. Serochkin, S. Shilov, A. Shalyugin, Yu. Usov Joint Institute for Nuclear Research, Dubna, Russia

J. Ban, D. Bruncko, R. Chytracek, A. Jusko, E. Kladiva, P. Strizenec Institute of Experimental Physics of the Slovak Academy of Sciences, Kosice, Slovakia

V. Gaertner, S. Hiebel, M. Hohlfeld, K. Jakobs, L. Koepke, E. Marschalkowski ${ }^{2}$, D. Meder, R. Othegraven, U. Schaefer, J. Thomas, W. Walkowiak ${ }^{3}$, C. Zeitnitz Institut für Physik der Universität Mainz, Mainz, Germany

\author{
C. Leroy, R. Mazini, R. Mehdiyev ${ }^{1}$ \\ Université de Montréal, Montréal, Canada
}
A. Akimov, M. Blagov, A. Komar, A. Snesarev, M. Speransky, V. Sulin, M. Yakimenko ${ }^{4}$
Lebedev Institute of Physics, Academy of Sciences, Moscow, Russia

M. Aderholz, H. Brettel, W. Cwienk, B. Dulny, J. Fent, A. Fischer, W. Haberer, J. Huber, R. Huber, A. Karev ${ }^{5}$, A. Kiryunin ${ }^{6}$, T. Kobler, L.Kurchaninov ${ }^{6}$,

H. Laskus, M. Lindenmayer, P. Mooshofer, H. Oberlack, D. Salihagic ${ }^{7}$, P. Schacht, H. Stenzel, D. Striegel, W. Tribanek Max-Planck-Institut für Physik, Munich, Germany

\footnotetext{
${ }^{1}$ On leave of absence from IP, Baku, Azerbaijan

${ }^{2}$ Now at Dialog Semiconductor GmbH, Munich, Germany

${ }^{3}$ Now at SCIPP, University of California, Santa Cruz, California, USA

${ }^{4}$ deceased

${ }^{5}$ On leave of absence from JINR, Dubna, Russia

${ }^{6}$ On leave of absence from IHEP, Protvino, Russia

${ }^{7}$ On leave of absence from University of Podgorica, Montenegro, Yugoslavia
} 
S. Chekulaev, S. Denisov, M. Levitsky, A. Minaenko, G. Mitrofanov,

A. Moiseev, A. Pleskatch, V. Sytnik ${ }^{8}$

Institute for High Energy Physics, Protvino, Russia

P. Benoit, K.W. Hoyle, A. Honma ${ }^{9}$, R. Maharaj, C.J. Oram,

E.W. Pattyn, M. Rosvick, C. Sbarra ${ }^{10}$, H-P. Wellisch ${ }^{9}$, M. Wielers

TRIUMF, Vancouver, Canada

P.S. Birney, M. Dobbs, M. Fincke-Keeler, D. Fortin, T.A. Hodges, R.K. Keeler, R. Langstaff, M. Lefebvre, M. Lenckowski, R. McPherson ${ }^{11}$, D.C. O’Neil ${ }^{12}$ University of Victoria, Victoria, Canada

D. Forbush, P. Mockett, F. Toevs

University of Washington, Seattle, USA

H.M. Braun, J. Thadome

University of Wuppertal, Wuppertal, Germany

October 23, 2001

accepted for publication in Nucl. Instr. and Meth. A

\begin{abstract}
Modules of the ATLAS liquid argon Hadronic End-cap Calorimeter (HEC) were exposed to beams of electrons, muons and pions in the energy range $6 \mathrm{GeV} \leq E \leq 200 \mathrm{GeV}$ at the CERN SPS. A description of the HEC and of the beam test setup are given. Results on the energy response and resolution are presented and compared with simulations. The ATLAS energy resolution for jets in the end-cap region is inferred and meets the ATLAS requirements.
\end{abstract}

\footnotetext{
${ }^{8}$ Now at University of California, Riverside, USA

${ }^{9}$ Now at CERN, Geneva, Switzerland

${ }^{10}$ Now at Istituto TESRE-CNR, Bologna, Italy

${ }^{11}$ Fellow of the Institute of Particle Physics of Canada

${ }^{12}$ Now at Michigan State University, Lansing, USA
} 


\section{Introduction}

In the ATLAS detector the hadronic liquid argon end-cap calorimeter (HEC) [1],[2] covers the pseudorapidity range $1.5<\eta<3.2$. The reconstruction of jets in the forward region and the measurement of the missing transverse energy are driving the requested performance parameters. To fully exploit the physics potential of ATLAS, an energy resolution for jets of typically $\sigma(E) / E=\frac{50 \%}{\sqrt{E(G e V)}} \oplus 3 \%$ is required. Liquid argon (LAr) was chosen as the active medium for its robustness against the high radiation levels present in this $\eta$ region.

In this paper we describe the calibration results obtained from the beam exposure of six calorimeter modules from the series production. Following a phase of testing prototypes and modules from the pre-series production [3], [4], [5], [6], [7], these runs have started in June 2000. They are part of the standard quality control procedure during the construction of the full HEC calorimeter.

The outline of the paper is as follows: In section 2 we review the design of the hadronic end-cap calorimeter including the amplifier electronics located on the calorimeter. In section 3 we describe the beam test setup including the read-out electronics and the calibration system. The simulation and the main features of the data analysis are given in section 4 . Finally in section 5 we discuss the beam test results for electrons, muons and pions and give an outlook for the jet measurement in ATLAS.

\section{The ATLAS Hadronic End-cap Calorimeter}

\subsection{Design Requirements}

The efficient tagging and reconstruction of forward jets associated with the production of heavy Higgs bosons sets the constraints for the energy resolution: the guideline for the energy resolution of jets is $\sigma(E) / E=\frac{50 \%}{\sqrt{E(G e V)}} \oplus 3 \%$. The transverse granularity is mostly driven by the aim to reconstruct the decay $W \rightarrow$ jet + jet at high $p_{T}$ : a granularity of $\Delta \eta \times \Delta \phi=0.1 \times 0.1$ is needed for the region $|\eta|<2.5$ and $\Delta \eta \times \Delta \phi=0.2 \times 0.2$ beyond $|\eta|=2.5$. The desired linearity of the energy response measured has to stay within $2 \%[2]$. Total energy containment up to the highest energies as well as acceptable low background in the muon chambers require a calorimeter thickness of at least 10 interaction lengths $(\lambda)$ including the electromagnetic calorimeter in front of the HEC. Fast electronics is needed in order to keep the pile-up low. On the other hand a shorter shaping time increases the noise contribution. Optimally the electronic noise should be comparable to or less than the signals from the expected pile-up at the highest LHC luminosity. In addition, the noise has to be low enough to allow for the identification of spatially well isolated muons. The speed of the response has to be fast enough to enable a bunch crossing identification with high efficiency. Last but not least long term reliability as well as cost efficiency are constraints imposed on any design option chosen. 


\subsection{Description of the Hadronic End-cap Calorimeter}

The hadronic end-cap calorimeter [1],[2] is a liquid argon sampling calorimeter with flat copper absorber plates. It shares the two end-cap cryostats together with the electromagnetic and forward calorimeters. The liquid argon technology has been chosen for its ability to cope with a high radiation environment and allows a simple and cost-effective design. The HEC is structured in two wheels, the front HEC1 and rear HEC2 wheel, placed in the cryostat behind the electromagnetic calorimeter wheel.

Figure 1 shows an artist's view of a HEC module: seven tie rods, made from stainless steel, maintain the overall mechanical structure. Annular spacers define $8.5 \mathrm{~mm}$ gaps between the absorber plates. Connecting bars at the outer and inner circumference between individual modules are used to form the final wheel structure. Each wheel has an outer diameter of about $4 \mathrm{~m}$. The length of HEC1 (HEC2) is $0.82 \mathrm{~m}(0.96 \mathrm{~m})$. The thickness of the copper absorber plates is $25 \mathrm{~mm}$ for HEC1 and $50 \mathrm{~mm}$ for HEC2, with the first plate being half of this normal thickness in either case. Each wheel is made out of 32 identical modules. The weight of HEC1 is $67 \mathrm{t}$ and that of HEC2 is $90 \mathrm{t}$.

In total 24 gaps for HEC1 and 16 gaps for HEC2 are instrumented with a read-out structure. Longitudinally they are read out as segments of 8 and 16 gaps for HEC1 and 8 and 8 gaps for HEC2. The read-out structure is based on the principle of an electrostatic transformer [8] (EST, see Figure 2). For each gap it consists of a central board, which contains the read-out electrode pads (PAD board), and two boards that are part of the EST structure (EST boards). Each EST board is made of a layer of insulator (polyimide) sandwiched between two high resistive layers (HRL) that are connected to ground or high voltage $(\mathrm{HV})$. The polyimide-based HRL material has a typical resistivity of $0.5 \mathrm{M} \Omega / \square$. The PAD board is made of a copper read-out electrode sandwiched between two insulators and covered on both outside faces with a HRL, like the EST board. Thus in total a four subgap structure with one PAD and two EST boards completes the electrostatic transformer structure. This yields in total 96 subgaps for HEC1 and 64 subgaps for HEC2. The high resistivity of the HRL yields a natural protection for the preamplifiers from potentially dangerous sparks in the argon gaps. In addition it provides a simple distribution of the HV or ground across the area of the full board. The space between the electrodes is maintained using honeycomb mats. The EST structure allows for a smaller gap design without increasing the overall capacitance. It decreases the required HV for a given electric field and shortens the drift time. The low capacitance enables a fast signal rise and keeps the electronic noise low.

In order to limit the capacitance seen by a single preamplifier, only two gaps are ganged together at the pad level. A stripline connector of $50 \Omega$ impedance running across the longitudinal section and coaxial cables running in between the sections carry the signal to the preamplifier boards located at the wheel periphery (see Figure 1). These signals are amplified and summed employing the concept of "active pads": the signals from two consecutive pads are fed into separate preamplifiers (based on highly integrated GaAs electronics, see below). The output signals from typically four preamplifiers are summed together within the same preamplifier chip. A buffer stage drives the output signal up to the cold-to-warm feedthroughs. The use of cryogenic GaAs preamplifiers and summing amplifiers provides the optimum signal-to-noise ratio for the HEC. The total number of 
read-out channels for a single $\phi$-wedge consisting of a HEC1 and a HEC2 module is 88 .

Modules are stacked at four institutes, from sub-components manufactured in industry and institutes. Manufactured modules are shipped to CERN. Careful Quality Control (QC) is required to assure a uniform conformance to design. Central to this QC are mechanical and electrical tests upon receipt of a module at CERN, followed by a cold test or a particle beam test. An eighth of all modules are beam tested. All QC data are stored in a database which is available to all institutes involved. All important measurements, for example the total module thickness, are stored in this database. Figure 3 shows a histogram of the module thickness acheived today in the 20 HEC1 and 13 HEC2 modules produced.

Copper plates are machined in industry and several institutes. The front module plates are manufactured from quarter hard cold rolled copper to provide sufficient strength, while the rear module plates which are twice as thick and therefore about eight times stiffer are manufactured from hot rolled copper. Each batch from the rolling mill had a sample produced to allow for the copper density and strength to be checked. Every production facility had its machining and cleaning method qualified by producing a sample which was carefully checked for argon poisoning. Care had to be taken with the cold rolled copper to obtain the flatness tolerance of $0.5 \mathrm{~mm}$ because surface machining relieved stresses that could cause significant bowing. The $0.25 \mathrm{~mm}$ flatness tolerance on the rear plates was more easily obtained. The plate thickness tolerance is $\pm 0.05( \pm 0.10) \mathrm{mm}$ for the front (rear) plates. This tolerance can, during module stacking, cause an excessive buildup in the height of the module. This is corrected for during stacking by using slightly larger or smaller $8.5 \mathrm{~mm}$ gap defining annular spacers between the absorber plates to correct for any tolerance buildup.

Both PAD and EST boards are manufactured in the institutes. These electrodes are manufactured from four main sub-components: $75 \mu \mathrm{m}$ sheet polyimide, $25 \mu \mathrm{m}$ sheet polyimide loaded with carbon with a typical resistivity of $0.5 \mathrm{M} \Omega / \square$, sheet glue, and copper clad polyimide etched with the electrode read-out structure. The electrodes are pie shaped and are about $1.6 \mathrm{~m}$ long and $0.4 \mathrm{~m}$ wide at the wide end. In view of these dimensions care has to be taken when cutting polyimide materials due to the variation in dimension with humidity. Cutting, which is accomplished with a steel rule die (prefabricated high precision cutting form), is undertaken only when the relative humidity is in the $(45 \pm 10) \%$ range. Manufacture of the electrodes is made following standard industry practices using a high pressure press and an oven. Two electrical connections are made to every high resistive layer to provide connection redundancy, and to provide a more direct path for the significant currents that will be produced in the high eta region of the detector during high luminosity operation of the LHC. Alignment of materials in the electrodes is achieved at the $0.3 \mathrm{~mm}$ level. The electrodes are spaced by $1.85 \mathrm{~mm}$ insulating honeycomb mats. These mats are cut using a steel rule die. Every mat has its thickness measured, and every 20 th mat is weighed. In this way the signal reduction due to replacement of the liquid argon by honeycomb is established. Boards containing glue from every batch of glue are tested in a high radiation environment, equivalent to 10 years of LHC operation. Subsequent to this irradiation the argon poisoning and peel strength is checked [9]. No significant deterioration was detected. In this way every batch of glue is qualified for use in the ATLAS experiment. 


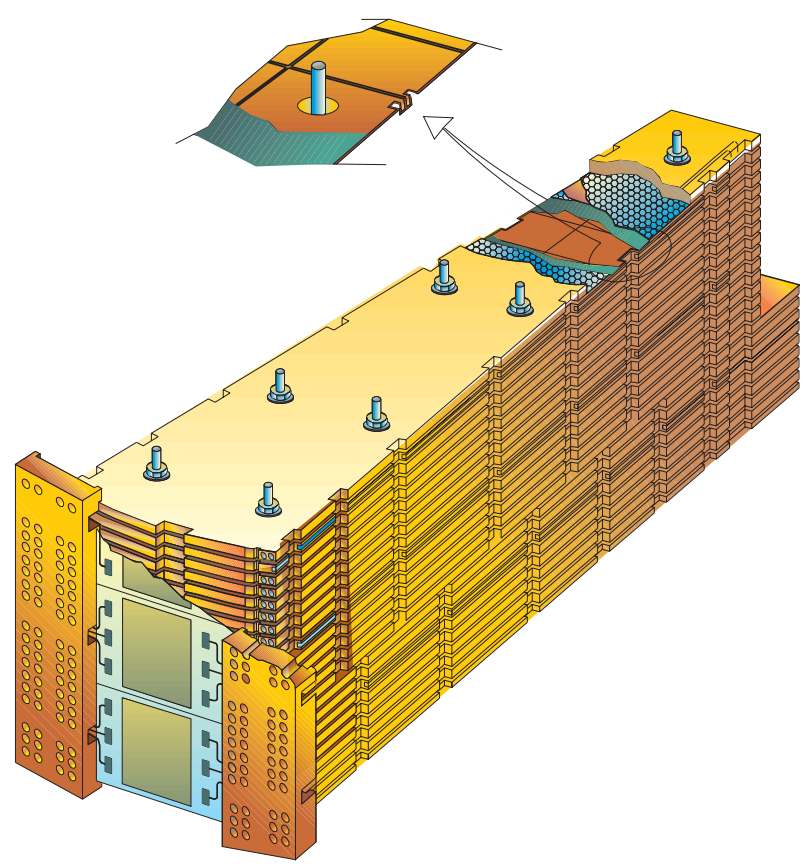

Figure 1: Artist's impression of a module, with cutaway showing the read-out structure.

Every module is tested at the production site for 2 weeks at high voltage $(2 \mathrm{kV})$. This procedure tests for any residual solvent bubbles that might be in the $75 \mu \mathrm{m}$ sheet polyimide. The modules are then wrapped and shipped to CERN. Upon receipt at CERN modules undergo detailed electrical and mechanical QC tests, and then are cold tested. Modules are accepted from the cold test if they have one or no short. The shorted subgap, if present, is disconnected reducing the signal of the affected tower by $1 / 32$ nd or $1 / 64$ th depending on the location. The module is then stored for subsequent assembly into wheels.

\subsection{Cold Front-end Electronics}

The read-out electronics as well as the calibration electronics used in the beam test setup are very similar to the electronics to be used in the final ATLAS scheme. The read-out chain is schematically shown in Figure 4.

The ionization signal from the liquid argon gap (or the calibration pulse) propagates via coaxial cables to the preamplifiers, where the signals from successive gaps are summed to one output channel. This output signal is then carried to the front-end board outside the cryostat.

A detailed description of the HEC front-end electronics can be found in two forthcoming papers [10],[11]. Here we summarize the main characteristics which are important for the analysis of the beam test data (see Table 1). The detector capacitance $C_{D}$ affects the signal rise time, amplitude and signal to noise ratio. For different pads $C_{D}$ varies from $20 \mathrm{pF}$ to $400 \mathrm{pF}$ giving a wide distribution for the rise time and noise. The ionization current depends on the electrical field in the LAr gap. The values in Table 1 correspond to the nominal value of $1800 \mathrm{~V}$ for the high voltage. 


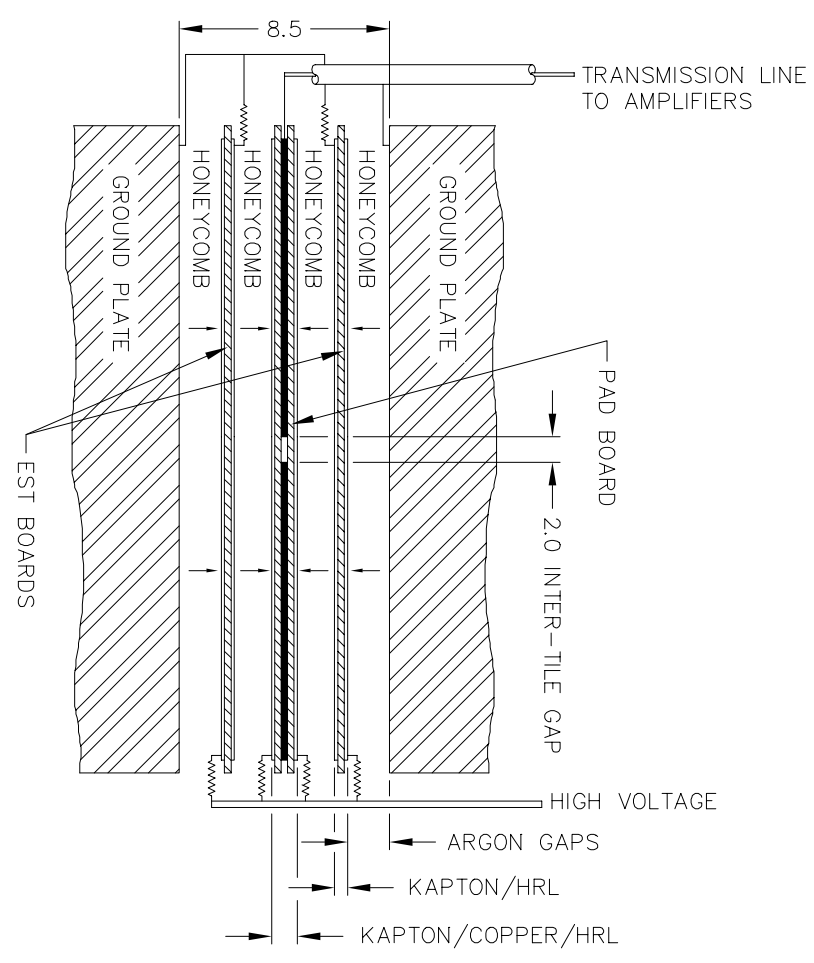

Figure 2: Schematic of the arrangement of the read-out structure in the $8.5 \mathrm{~mm}$ inter-plate gap.

The preamplifier ASIC is made by the GaAs TriQuint QED-A process. This technology has been chosen because it offers excellent high frequency performance, low noise, stable operation at cryogenic temperatures and radiation hardness. One chip contains eight identical preamplifiers and two drivers, giving the possibility to sum signals according to the physics requirements. The preamplifiers are placed on the outer radius of the calorimeter wheel inside the cryostat. Five preamplifying and summing boards (PSB) process signals from one HEC $\phi$-wedge (made of one HEC1 and one HEC2 module). They have 67 chips with 88 output channels in total. All signal and calibration cables are high quality radiation resistant (made of PEEK) $50 \Omega$ coaxial cables. The cable length from the pad to the preamplifier input is $\sim 0.2 \mathrm{~m}$ for the outer region and $\sim 2 \mathrm{~m}$ for the innermost part. The total length of the calibration cables in the beam test setup is $11.8 \mathrm{~m}$ (corresponding to $12.2 \mathrm{~m}$ in the final ATLAS detector) and the length of the signal cables is $8.3 \mathrm{~m}$ (corresponding to $8.7 \mathrm{~m}$ in the final ATLAS detector). Because of the substantial length of cables all effects due to signal attenuation and shape distortion have to be taken into account in the calibration procedure. The preamplifier and cable characteristics have been studied at room temperature and at liquid nitrogen temperatures. Their transfer function has been parameterized in the frequency domain by two poles and by one zero and two poles. Typical values of the parameters are given in Table 1. 


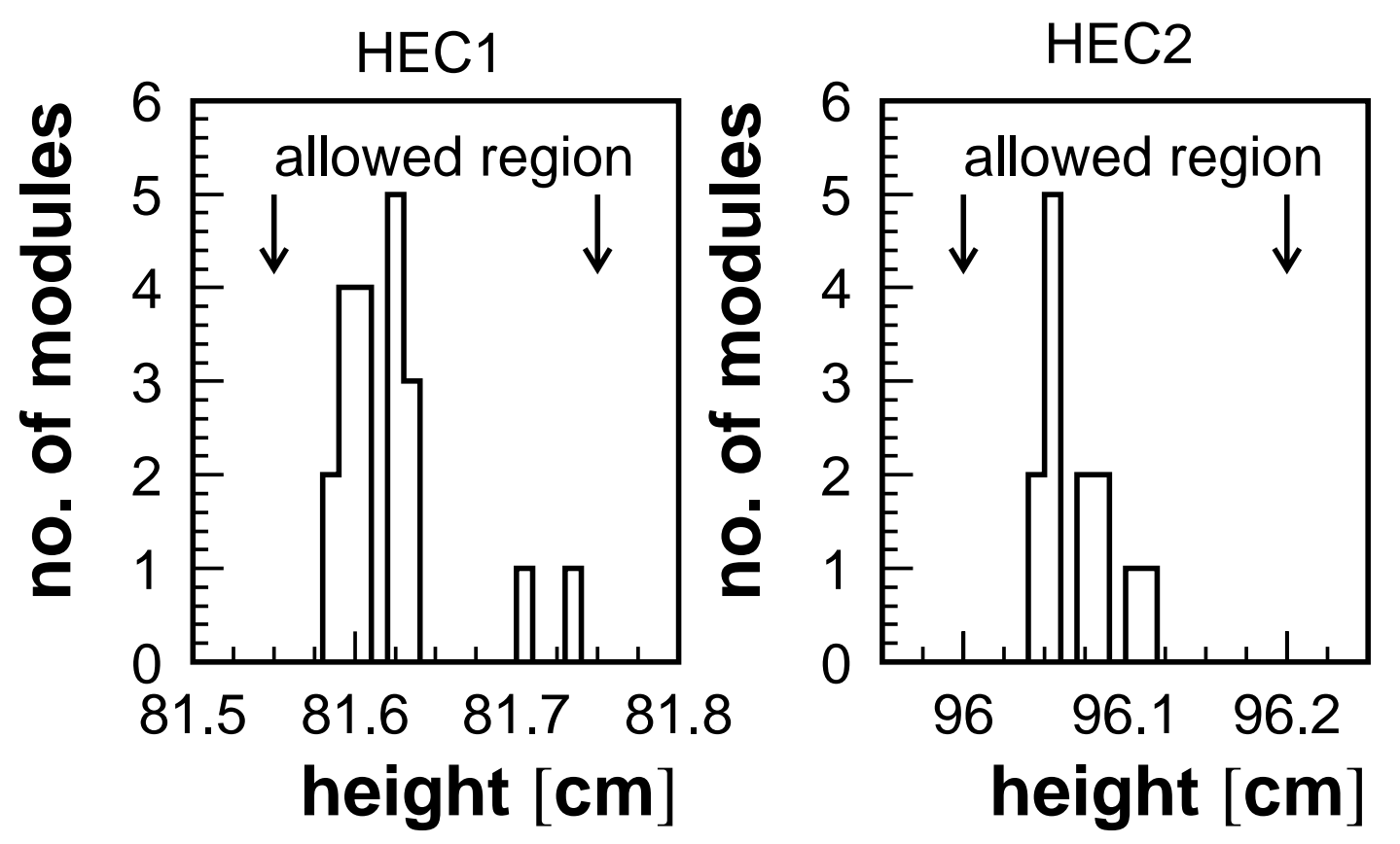

Figure 3: Histogram of the module height acheived to date in the 20 HEC1 and 13 HEC2 modules produced. 


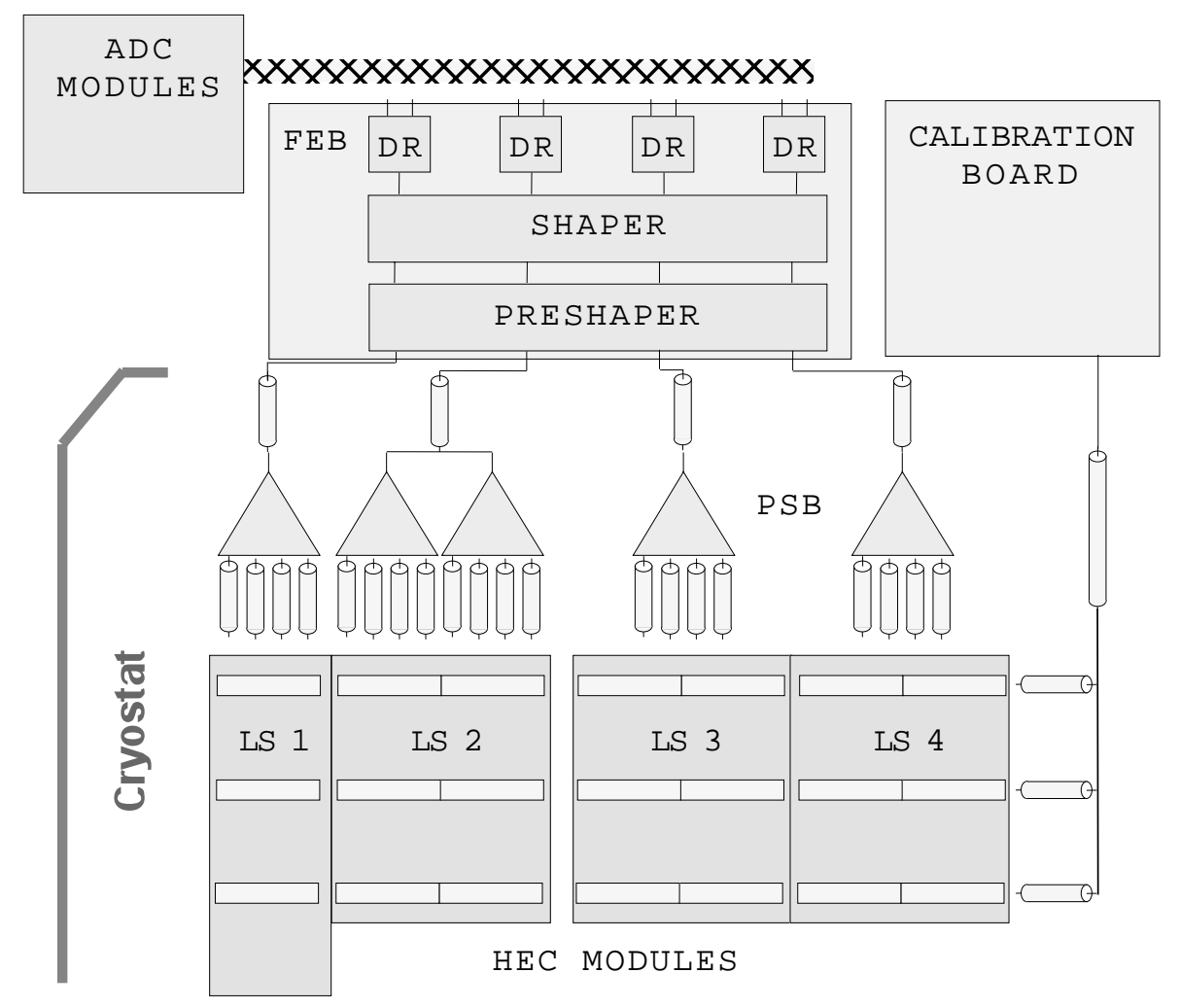

Figure 4: Schematic of the HEC beam test electronics, showing the longitudinal segments (LS), the preamplifying and summing boards (PSB), the front-end board (FEB), the FEB drivers (DR), the calibration board and the analog-to-digital converter (ADC) modules.

\section{Particle Beam Tests}

\subsection{General Beam Test Setup}

The concept and the technology used for the construction of the hadronic end-cap calorimeter have been verified in beam tests in the years 97-99 using either prototype modules or pre-production modules. The series ATLAS HEC modules are tested either in technical cold tests or in dedicated beam tests. These tests are part of the general quality control procedures to guarantee their successful operation in the ATLAS detector. The first two beam tests with series ATLAS HEC modules have been carried out in 2000 . In each beam test three $\phi$-wedges (each made of one HEC1 and one HEC2 module) of the HEC have been tested. Simulation studies show that this setup yields a total leakage of incident pion energy below $\sim 3.5 \%$ in the energy regime accessible. This rather low level of energy leakage allows a precise determination of the calorimeter performance parameters.

The beam tests have been carried out with a separated beam (H6) of the CERN SPS. It is a secondary or tertiary particle beam that provides hadrons, electrons or muons in the energy range $6 \mathrm{GeV} \leq E \leq 200 \mathrm{GeV}$. The beam intensity varies strongly with energy and particle type. Given the maximum trigger rate of 400 per burst that could be achieved, the 


\begin{tabular}{|c|c|c|c|}
\hline Chain component & parameters & values & transfer function \\
\hline Detector & $\begin{array}{l}\text { capacitance } \\
\text { cable length } \\
\text { drift time }\end{array}$ & $\begin{array}{c}C_{D}=20-400 \mathrm{pF} \\
0.2-2 \mathrm{~m} \\
\tau_{d r}=450 \mathrm{~ns}\end{array}$ & $\begin{array}{l}- \\
- \\
-\end{array}$ \\
\hline Preamplifier & $\begin{array}{c}\text { input imp. } \\
\text { integration } \\
\text { transimpedance } \\
\text { driver }\end{array}$ & $\begin{array}{c}R_{a}=50 \Omega \\
\tau_{a}=4-23 \mathrm{~ns} \\
R_{p}=0.75 \mathrm{k} \Omega \\
\tau_{d a}=4 \mathrm{~ns}\end{array}$ & $\frac{R_{p}}{\left(1+s \tau_{a}\right)\left(1+s \tau_{d a}\right)}$ \\
\hline Signal cable & $\begin{array}{c}\text { attenuation } \\
\text { zero } \\
\text { pole } 1 \\
\text { pole } 1\end{array}$ & $\begin{array}{c}a_{s}=0.965 \\
\tau_{z s}=24.5 \mathrm{~ns} \\
\tau_{o s}=1.2 \mathrm{~ns} \\
\tau_{p s}=28.5 \mathrm{~ns}\end{array}$ & $\frac{a_{s}\left(1+s \tau_{z s}\right)}{\left(1+s \tau_{o s}\right)\left(1+s \tau_{p s}\right)}$ \\
\hline Preshaper & $\begin{array}{c}\text { gain } \\
\text { zero } \\
\text { pole } 1 \\
\text { pole } 1\end{array}$ & $\begin{array}{c}G_{p}=6.5 \\
\tau_{p z}=0-20 \mathrm{~ns} \\
\tau_{i}=29 \mathrm{~ns} \\
\tau_{o}=2.5 \mathrm{~ns}\end{array}$ & $\frac{G_{p}\left(1+s \tau_{p z}\right)}{\left(1+s \tau_{i}\right)\left(1+s \tau_{o}\right)}$ \\
\hline Shaper & $\begin{array}{l}\text { gain } \\
\text { time }\end{array}$ & $\begin{array}{c}G_{s}=9.2 \\
\tau_{s}=13.5 \mathrm{~ns}\end{array}$ & $\frac{3.69 \cdot G_{s} s \tau_{s}}{\left(1+s \tau_{s}\right)^{3}}$ \\
\hline $\begin{array}{l}\text { Driver and } \\
\text { ADC cable }\end{array}$ & $\begin{array}{l}\text { pole } \\
\text { pole }\end{array}$ & $\begin{array}{l}\tau_{d f}=2 \mathrm{~ns} \\
\tau_{a c}=7 \mathrm{~ns}\end{array}$ & $\frac{1}{\left(1+s \tau_{d f}\right)\left(1+s \tau_{a c}\right)}$ \\
\hline
\end{tabular}

Table 1: The main electronic parameters of the detector and of the front-end electronics as well as the transfer functions used.

particle intensity has been kept typically below 2000 particles per burst. The general setup is shown in Figure 5. Using either three HEC1 or three HEC2 modules, a partial fraction of the HEC1 or HEC2 wheel respectively is assembled using the final wheel assembly technique. These HEC1 and HEC2 partial wheels are placed together in the beam test cryostat forming three full $\phi$-wedges of the HEC calorimeter. The segmentation of the HEC is pseudo-pointing in $\eta$ and pointing in $\phi$ in the ATLAS detector. In the beamtest the impact angle is constant, i.e. $90^{\circ}$ with respect to the front plane, yielding for the energy reconstruction of particles a somewhat larger number of channels than in ATLAS. Figure 6 shows a view of the two partial HEC wheels in the cryostat during the installation.

The cryostat with an inner diameter of $2.50 \mathrm{~m}$ and a fill height of LAr of $2.20 \mathrm{~m}$ can be moved horizontally by $\pm 30 \mathrm{~cm}$. The beamline bending magnet closest to the experimental zone allows to deflect the beam vertically by $\pm 25 \mathrm{~cm}$ at the front face of the cryostat. Thus a scanning area of typically $60 \times 50 \mathrm{~cm}^{2}$ is available for horizontal and vertical scans. A beam window with reduced wall thickness $(5.5 \mathrm{~mm}$ stainless steel in total and a diameter of $60 \mathrm{~cm}$ ) of the cryostat minimizes the dead material for particles prior to hitting the calorimeter within this area. In addition, a LAr excluder in front of the modules is installed for the same reason.

Figure 5 shows the location of the trigger and veto counters. The beam trigger is derived from the signals of a differential Cherenkov counter for particle identification up 


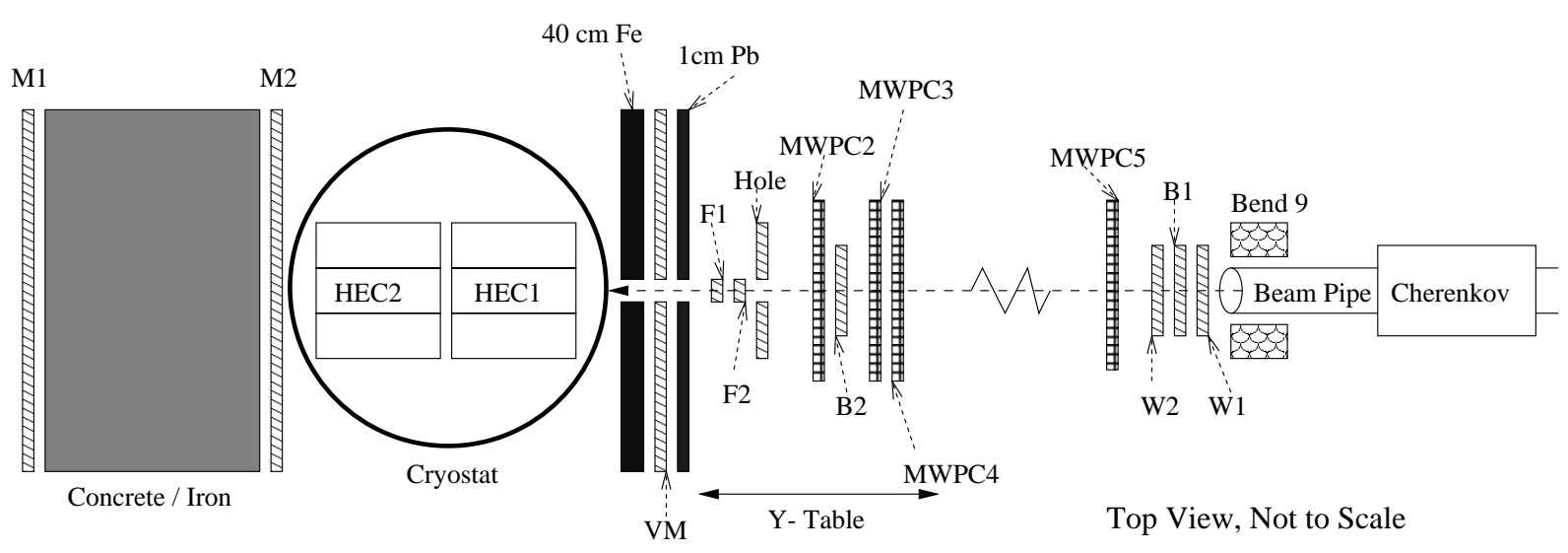

Figure 5: The setup used for data taking in the beam tests. The trigger is defined by the scintillation counters B1, F1 and F2 and the scintillation counter walls VM, M1 and M2

to $80 \mathrm{GeV}$, of one upstream scintillation counter (B1), of three scintillator walls (VM, M1, M2), and of two scintillation counters for fast timing (F1, F2 with $\sigma=70 \mathrm{ps}$ ). Impact position and angle of a beam particle have been determined using four multiwire proportional chambers (MWPC) with two planes (vertical and horizontal) per chamber. The wire spacing is $1 \mathrm{~mm}$ (MWPC2, MWPC3, MWPC4) and $2 \mathrm{~mm}$ (MWPC5) respectively. Veto counters (VM) are used to reject particles from the beam halo. To also veto photons with high efficiency, an additional lead layer has been added in front of the veto wall. Downstream of the cryostat an iron absorber with scintillation counter walls in front (M1) and behind (M2) is used for triggering or tagging muons.

\subsection{Calorimeter Operation}

The cryogenic system has been operated from the common ATLAS beam test cryogenic system [12].

As described above, each LAr gap contains an electrostatic transformer structure (EST), which effectively divides the gap into four sub-gaps. The HV for these four subgaps is supplied by individual HV lines, which feed each longitudinal read-out segment separately. Typically not more than one HV line out of 48 had to be disconnected because of a HV problem in one sub-gap. With a total number of 480 sub-gaps this amounts to a sub-gap failure rate of $0.2 \%$. It has been shown that the energy resolution is nevertheless recoverable - except for an increased noise contribution - by using simple multiplicative depth correction factors to offset the reduction of the signal in read-out segments affected. Because the distribution of HV lines is matched to the individual read-out segments the individual shower fluctuations are measured correctly, thus enabling a recovery of the energy resolution in case of minor HV problems.

Runs (typically 30000 triggers) with random triggers only have been taken regularly to analyze the electronics performance with respect to electronic noise and pedestal stability. In addition, random triggers have been taken in parallel with real particle triggers (typically at the level of $5 \%$ ) to enable a continuous monitoring of these quantities and 


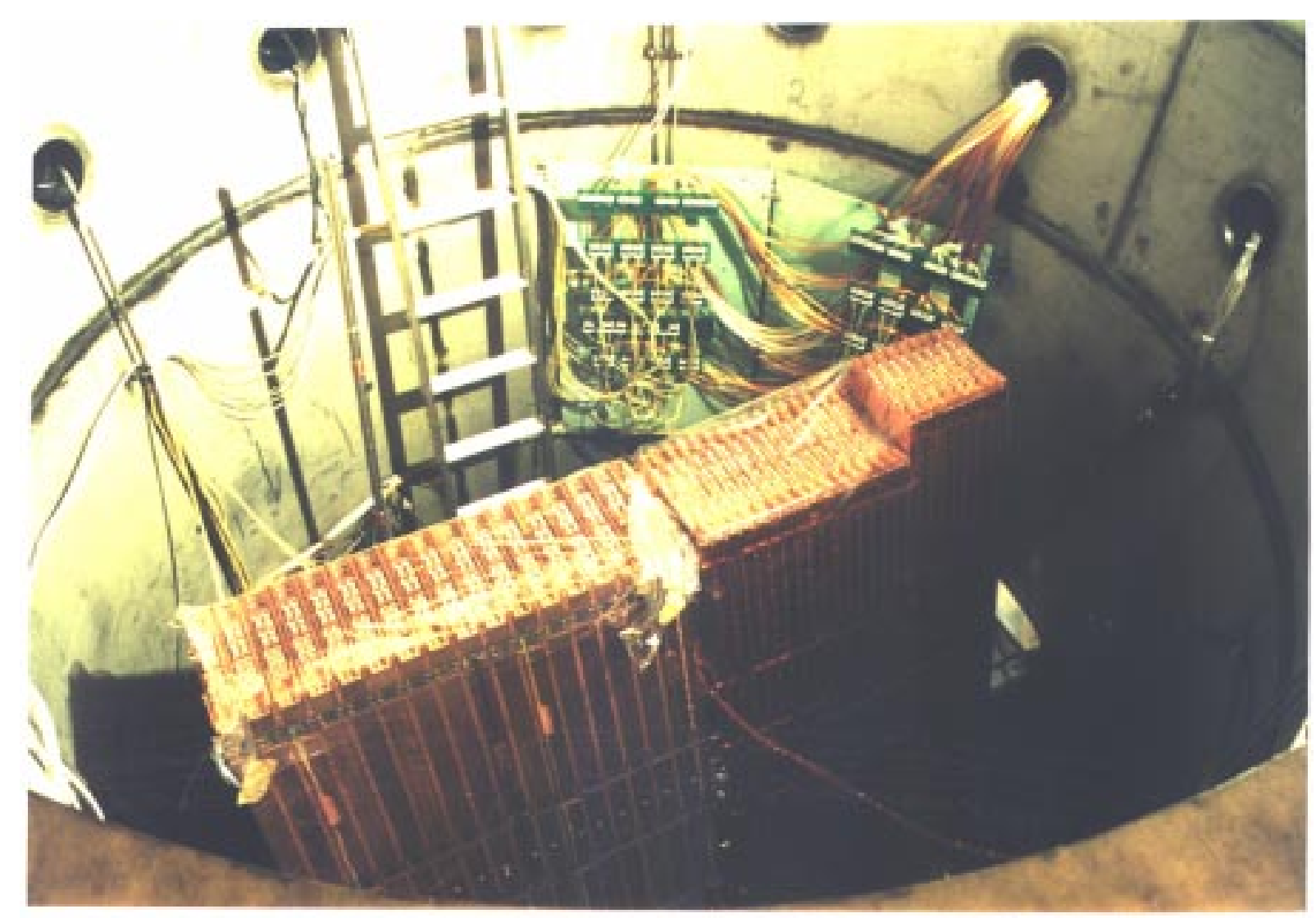

Figure 6: View of the setup of the HEC1 and HEC2 partial wheels in the cryostat. Each partial wheel is built out of three modules, the final calorimeter will have 32 modules per wheel.

to study possible differences between "in burst" and "out of burst" random triggers.

Data have been collected in two run periods, with typically 1000 runs per run period and about 20000 triggers per run. The data quality has been monitored during data taking by running the online version of the offline reconstruction task [13]. All relevant quantities such as signal response in the calorimeter, its spatial distribution and time evolution, the electronics performance, the MWPC response and efficiency as well as the trigger quantities have been continuously controlled during data taking. 


\subsection{Read-out Electronics and DAQ}

\subsubsection{Read-out Electronics}

As shown in Figure 4 the output signal of the cold summing amplifiers is carried to the front-end board (FEB) outside the cryostat where the analog signal shaping is performed. The crate with the FEB's is installed directly on the cryostat feedthrough, extending thus the Faraday cage of the cryostat. The rise time compensation is performed in the preshapers and the final pulse shape is formed by the shapers. The signal waveform is digitized by ADC modules and the data are transferred via the VME bus to the data acquisition system.

Three FEB's with 128 channels per board process the signals from up to four HEC $\phi$-wedges. The FEB used in the beam test is a prototype board of the final ATLAS board (only the analog part is present). The preshapers equalize the signal rise time by the pole-zero cancellation method. Each individual channel is adjusted to the expected value of the detector capacitance, giving 14 different time constants in total. An additional integration is introduced in order to reach a peaking time of $50 \mathrm{~ns}$ at the output of the chain. The signal is also inverted and amplified in the preshaper in order to adapt to the working range of the shaper. The amplification for the HEC2 modules is a factor of two higher to compensate for the smaller sampling ratios in these calorimeter modules. One preshaper hybrid contains four channels corresponding to the four longitudinal HEC channels with the same $\eta$ and $\phi$ location in ATLAS. The final signal shape is formed by a $R C^{2}-C R$ monolithic shaper with a time constant of about $14 \mathrm{~ns}$ and a gain of ten. One FEB is equipped with 32 four-channel chips. The shaper chips are of the final ATLAS version, the detailed information can be found in [14]. The unipolar signals from the shaper output are converted to differential signals by the FEB drivers. This component is specific to the beam test setup and will not be present in the final ATLAS scheme. The signals from the FEB are routed to the ADC modules via $3 \mathrm{~m}$ long twisted pair cables.

All parameters of the components of the electronic chain have been determined from measurements either in the laboratory or in the real beam test setup. To obtain a detailed description of the signal shape, the integration in the driver and the distortion in the cable are also taken into account (see the last row of Table 1). The numbers presented in Table 1 are averaged values. The overall transfer function of the signal chain is the product of all functions in the last column. This function consists of two zeros and nine poles, and it is used to describe the calibration and ionization signals.

An event trigger stops the digitization and outputs of the ADC chip are disabled. The signals from the FEB boards are digitized by twelve 32-channel ADC modules, installed close to the cryostat. The modules are VME-9U boards. Each motherboard contains eight plug-in units with four digitizing channels. The signal is sampled every 25 ns using a 12-bit ADC chip (Burr-Brown Model ADS800) and stored into a 256-cells circular-RAM with 10 ns access time. As the next step the CPU reads the RAM content in a block-transfer mode through the VME Bus. The ADC input signal range of $\pm 2 \mathrm{~V}$ yields a conversion factor of $1 \mathrm{mV}$ per ADC count. Each line of the differential input signal is terminated by a $50 \Omega$ resistor, connected to an adjustable voltage level. This level is used to shift the pedestal level to $\sim 800$ ADC-counts in order to get the negative part of the signal digitized as well. The ADC has an integral nonlinearity of \pm 2 LSB and 
a differential linearity error of \pm 0.6 LSB.

\subsubsection{Data Acquisition System}

The data acquisition is based on a multi-crate VME system, connected by a VME interconnect bus VIC [15]. The block diagram is shown in Figure 7. The ADC modules are read via their VME bus interface by a single RISC CPU module (MIPS 3000 based CES RAID, running the real time system CDC EP/LX [16]). In addition, the relative time of the pretrigger to the $40 \mathrm{MHz}$ ADC clock is read from a TDC module. The beam information, i.e. the MWPC electronics (PICOS-II system) and pattern units for the scintillation counters, is read via a CAMAC system. During one SPS burst the data are buffered in the RISC CPU, then copied to an HP workstation (type HP 9000/748) and recorded on disk. Given an event size of $\sim 13$ kbyte, resulting from the read-out of 16 time samples per ADC channel, the VME access to the ADC data via the VIC bus and the CAMAC read-out limit the data rate to approximately 300 events per SPS burst $(\sim 2.5 \mathrm{~s})$. The workstation, in addition to its role as a data recorder, serves as online data monitor providing histograms and performance checks. The data are sent to CERN's HPSS storage library asynchronously to the data acquisition through a network file copy. 


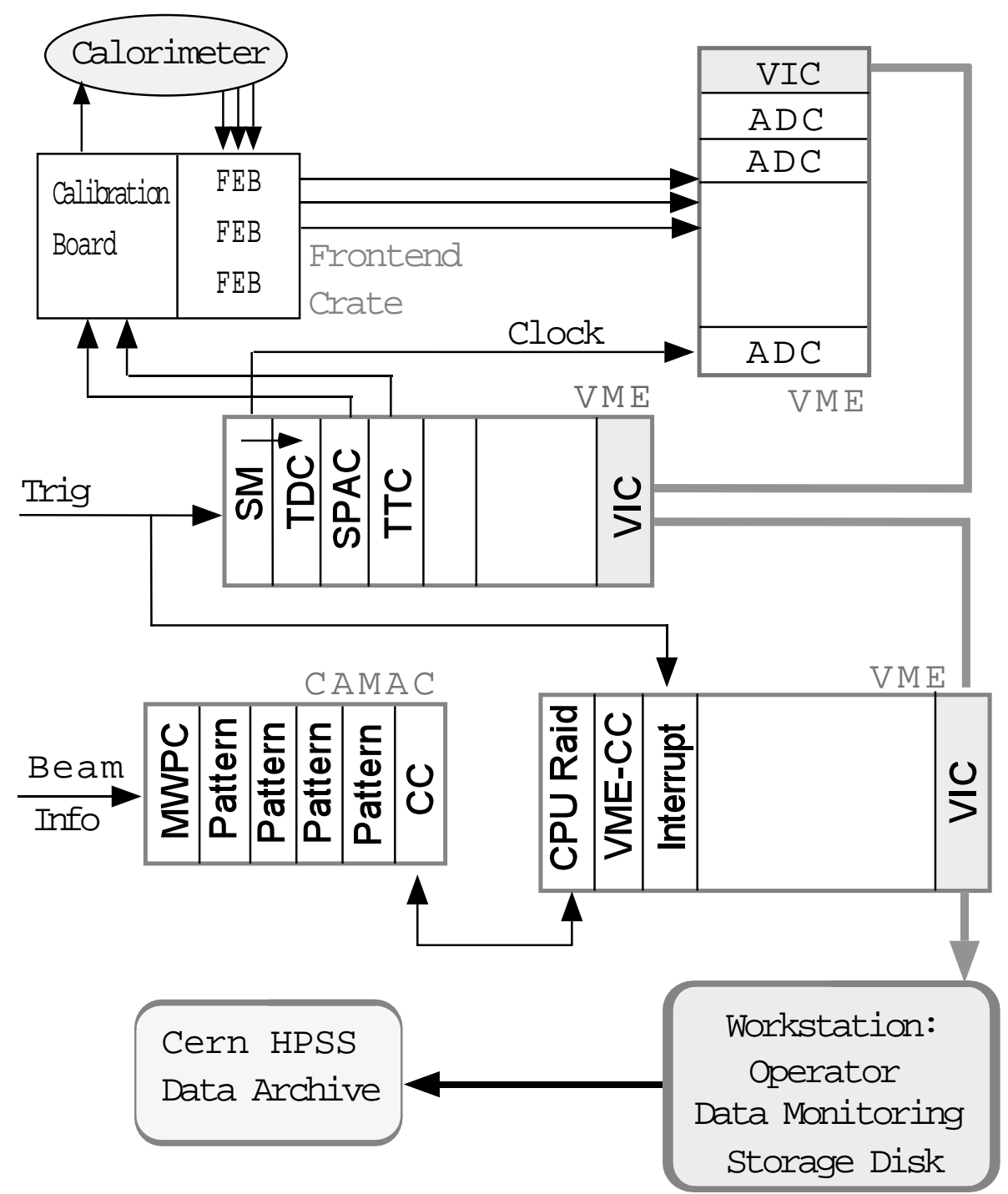

Figure 7: Block diagram of the data acquisition system. 


\subsection{Calibration and Signal Processing}

The main purpose of the calibration of the electronic chain is to equalize the gains of all channels and to correct any nonlinearity of the response. Calibration errors normally result in an increase of the constant term in the energy resolution. The system has to keep track of the timing stability and, if necessary, provide corrections. Another goal of the calibration is to measure the signal shape and the noise autocorrelation function. This information is used to produce weights for the digital filtering algorithm (see below). Finally the calibration system is used to determine the inter-channel cross-talk, which is important for an accurate energy reconstruction.

The calibration board contains 128 pulse generators and is installed in the front-end crate. This board is a pre-series module of the final ATLAS board [17]. The voltage pulse from one generator is guided through coaxial cables to a strip line board located close to the detector pads. Each generator signal is split into three on the calibration distribution board (placed at the back plane of each HEC module) in order to reduce the number of cables and feedthrough connectors. Each of these three signals pulses up to 16 preamplifiers via high precision calibration resistors located on the strip line board. In total 16 generators are used for one HEC $\phi$-wedge. The strip line board is mounted in parallel to the beam direction in notches in the copper plates, close enough to the pads, so that the delays of calibration pulses to different pads approximately mimic the particle signal timing. The calibration procedure is described in detail in [18]. It consists typically of four different steps: (i) measurement of gain and nonlinearity by pulsing all the channels with different pulse levels, (ii) measurement of signal shape by performing either delay scans (25 ns range with 1 ns steps) synchronous with the trigger, or, in asynchronous mode, determining the time by the TDC module, (iii) measurement of cross-talk by simultaneously pulsing one or a few generators and reading all neighbouring channels, and (iv) measurements of noise with all generators switched off. A minor change was made to the standard LAr calibration board, in order for it to be compatible with the overall beam test setup: the internal resynchronization of the pulse with the clock has been switched off. Instead a VME based module, called a Service Module (SM), has been used for the generation of the $40 \mathrm{MHz}$ clock and of the timing of the calibration pulse command. The SM also produces the start and stop signals for the TDC module, which measures the time delay between the trigger and the clock pulse. The communication with the calibration board is done via the VME based SPAC and TTC modules [19].

The steering software is a modular system, which practically allows any type of measurements either in the time of setting up and debugging the electronics or during standard calibration runs. The measured data are transferred via direct memory access (DMA) in VME from the ADC boards to the RAID computer and sent to the HP computer for storing. For this operation the CALTCP protocol is used, an OO protocol, which is based on TCP/IP and is highly optimized for speed [20]. The additional visualization tools are based to a large extent on a ROOT library [21].

For the amplitude reconstruction, the weighted sum of five signal samples (or four samples and pedestal) will be implemented in ATLAS [22, 23]. The same approach has been chosen for the beam test data analysis. The energy deposited in the read-out cell is 
calculated as

$$
E_{d}=\sum_{n=1}^{5} W_{n} U_{n}
$$

where $W_{n}$ are predefined weights and $U_{n}$ are the measured signal samples. The weights are determined by minimizing the noise to signal ratio and are calculated from the ionization signal shape and noise autocorrelation coefficients [22]. One of the important steps in the calibration analysis is the prediction of the particle waveform on the basis of the calibration shape measurements. This prediction is obtained by fitting the calibration signal with a model function and extracting two time constants: the preshaper integration time $\tau_{i}$ and the shaper time $\tau_{s}$. The particle signal shape is then calculated by a convolution of the electronic chain response with the triangular ionization current from a real signal. The model function for the calibration signal is obtained from the parameterization of all components of the calibration chain, shown (together with the full signal chain) in Figure 8. The parameterization is similar to the one used for the signal chain description. It is derived from oscilloscope measurements in the laboratory and in the beam setup. The functions and parameters are given in Table 2.

\section{CALIBRATION}
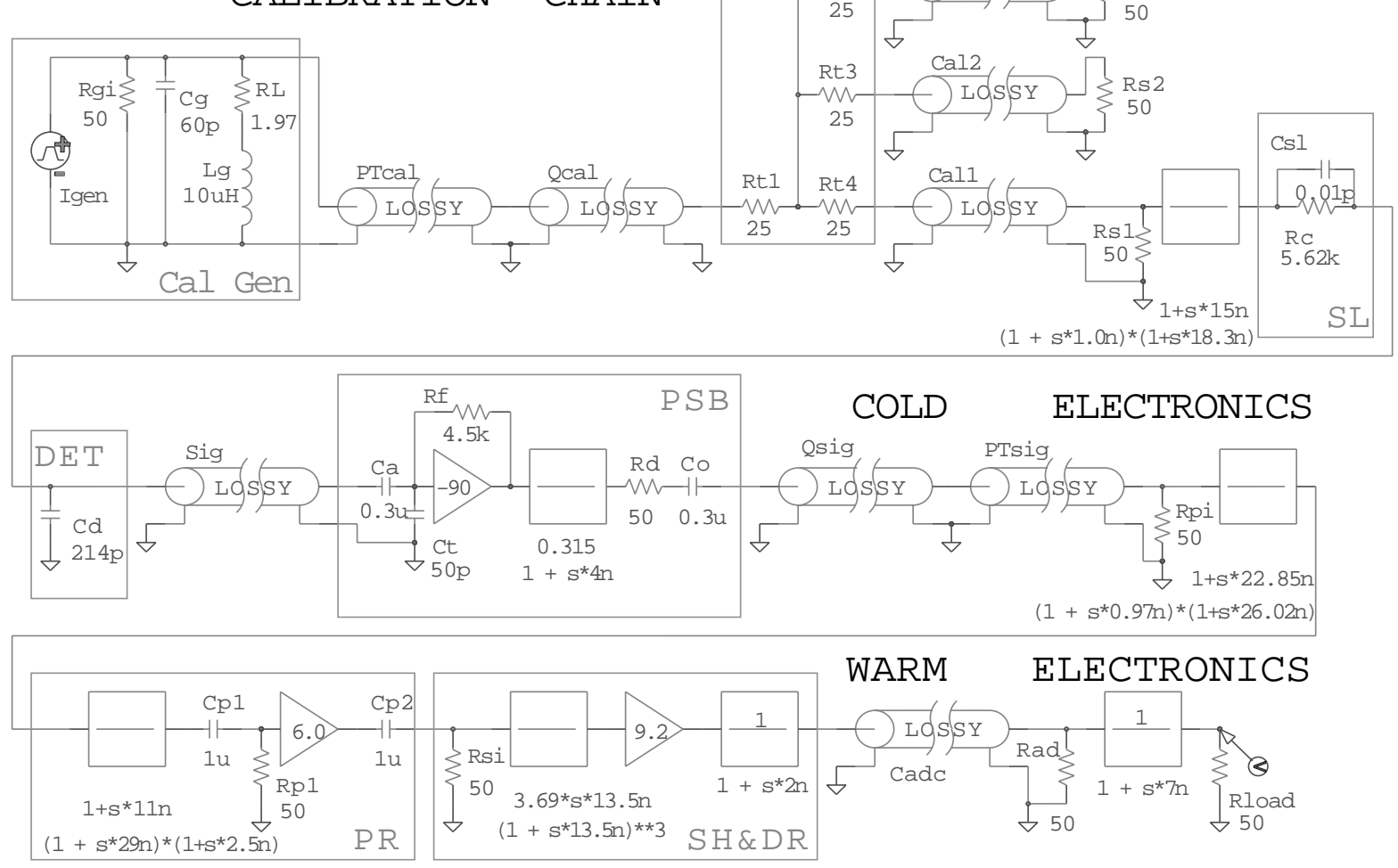

Figure 8: Schematics of the HEC electronic chain, showing the calibration generator board, the preshaper (PR), the shaper and FEB driver (SH\&DR) located in the warm and the the calibration distribution board (CDB), the strip line (SL), the detector gap (DET) and the preamplifier and summing board (PSB) located in the cold LAr. 


\begin{tabular}{|c|c|c|c|}
\hline Chain component & parameters & values & model function \\
\hline Generator & Decay time & $\tau_{d}=350 \mathrm{~ns}$ & $\frac{\alpha+s \tau_{d}}{s\left(1+s \tau_{d}\right)}$ \\
& step fraction & $\alpha=0.07$ & \\
\hline Cables & attenuation & $a_{c}=0.905$ & \\
& zero & $\tau_{z c}=18 \mathrm{~ns}$ & \\
& pole 1 & $\tau_{o c}=1.2 \mathrm{~ns}$ & $\frac{a_{c}\left(1+s \tau_{z c}\right)}{\left(1+s \tau_{o c}\right)\left(1+s \tau_{p c}\right)}$ \\
& pole 2 & $\tau_{p c}=21 \mathrm{~ns}$ & \\
\hline
\end{tabular}

Table 2: Parameters of the calibration signal and model functions.

In order to minimize the CPU time for the analysis, an analytical expression for the signal shape has been derived. The full function for the calibration signal in the frequency domain consists of 12 poles and 4 zeros, which would give an extremely complicated expression in the time domain. For the analysis of the data this function has been simplified by removing a few minor parts. The quality of the fit to the calibration signal and the ionization signal prediction are illustrated in Figure 9. Typical residuals (shown in the lower Figures) are $\pm 0.5 \%$ for the calibration pulse and $\pm 1.5 \%$ for the ionization signal. The fit is performed only for the positive part of the signal wave since only this region is used for the energy determination.

The transformation from ADC counts to current is accomplished using a third-order polynomial function. The related coefficients have been determined from the fit of the calibration amplitude ramp. The amplitude of the calibration pulse is determined by applying weights similar to the ones used in equation 1 . In this case the weights are derived from the calibration pulse shape and differ from the weights applied to the real ionization signal. The typical nonlinearity for the energy range accessible in the beam exposures is $\sim 1 \%$. But the calibration generators have some parasitic effects at low amplitudes [17], which makes a precise calibration in the region of the muon signal rather difficult. 

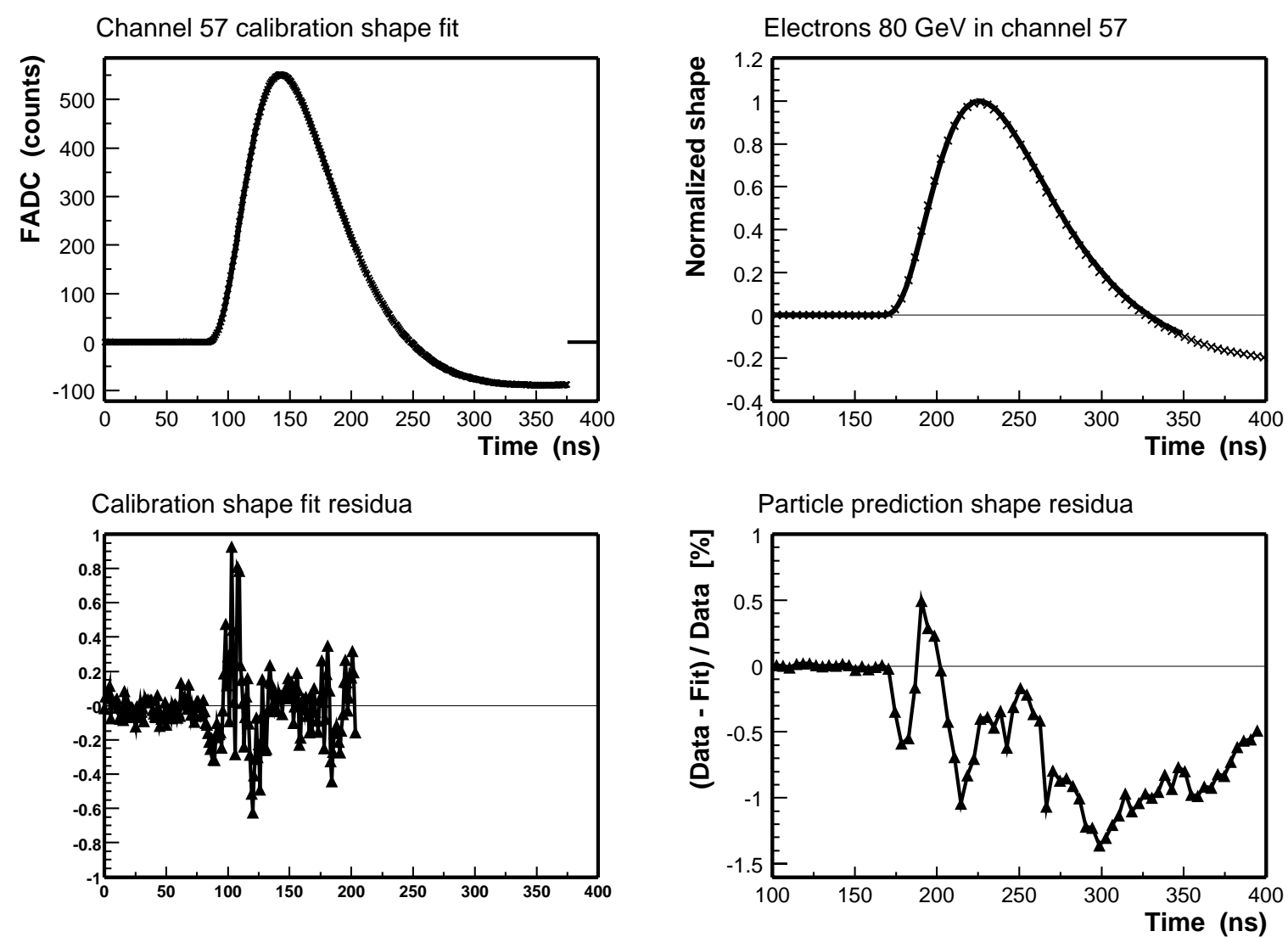

Figure 9: The calibration signal (left) and the prediction for the ionization signal (right) together with the residuals with respect to the fit (lower figures). 


\subsection{Liquid Argon Purity Monitoring}

The purity monitoring system utilizes two different radioactive sources which provide the means to ionize the liquid argon. The liberated charge is collected and the mean measured signal is used to determine the $\mathrm{O}_{2}$-concentration [24].

The first system consists of an ${ }^{241} \mathrm{Am}$ source which provides $5 \mathrm{MeV} \alpha$-particles. The range of $\alpha$-particles in the LAr is only approximately $100 \mu \mathrm{m}$, hence the ionization density is very high. The deposited charge is collected over a gap of $2 \mathrm{~mm}$ at an electric field strength of $12.5 \mathrm{kV} / \mathrm{cm}$. The collected charge for pure argon is about $5 \mathrm{fC}$. For an impurity caused by oxygen, the signal decreases nearly linearly with the oxygen concentration.

The second system employs a ${ }^{207} \mathrm{Bi}$ source. It provides mono energetic $1 \mathrm{MeV}$ electrons from an electron conversion process. The electrons have a mean range of $3 \mathrm{~mm}$ in LAr. The $6 \mathrm{~mm}$ wide LAr gap is separated by a Frisch-grid [24] into two compartments of $5 \mathrm{~mm}$ and $1 \mathrm{~mm}$ each, with an electrical field strength of $5 \mathrm{kV} / \mathrm{cm}$ and $25 \mathrm{kV} / \mathrm{cm}$, respectively. The electron deposits its energy in the $5 \mathrm{~mm}$ gap. The liberated charge drifts through the grid into the second compartment, which is attached to a charge sensitive amplifier. In this way the signal does not depend strongly on the track length or on the emission angle of the electron, resulting in an increased sensitivity to impurities. A quadratic dependence on the $\mathrm{O}_{2}$-concentration is expected.

Both cells are simultaneously read out by a single charge sensitive amplifier [25] which is located next to the cells in the LAr. The signals from the two sources are distinguished by their different sign (positive for the ${ }^{241} \mathrm{Am}$ cell and negative for the ${ }^{207} \mathrm{Bi}$ cell). From the ratio of the measured signals of the two cells the $\mathrm{O}_{2}$-contamination is estimated. In order to determine the absolute $\mathrm{O}_{2}$-concentration the signal ratio has been measured in a test cryostat as function of a known oxygen contamination. The systematic uncertainty of this measurement is about $30 \%$. The measured contamination over a period of 25 days during the beam test is shown in Figure 10. An increase of the oxygen contamination of approximately $1.6 \mathrm{ppb}$ per day has been observed, starting from about $12 \mathrm{ppb}$ at the beginning of the run period up to about $52 \mathrm{ppb}$ at the end of the run period. This rather small increase is attributed to intrinsic leaks in the cryogenic system. The visible variations of the measured contamination are due to a temperature dependence of the high voltage system for which no correction has been applied. 


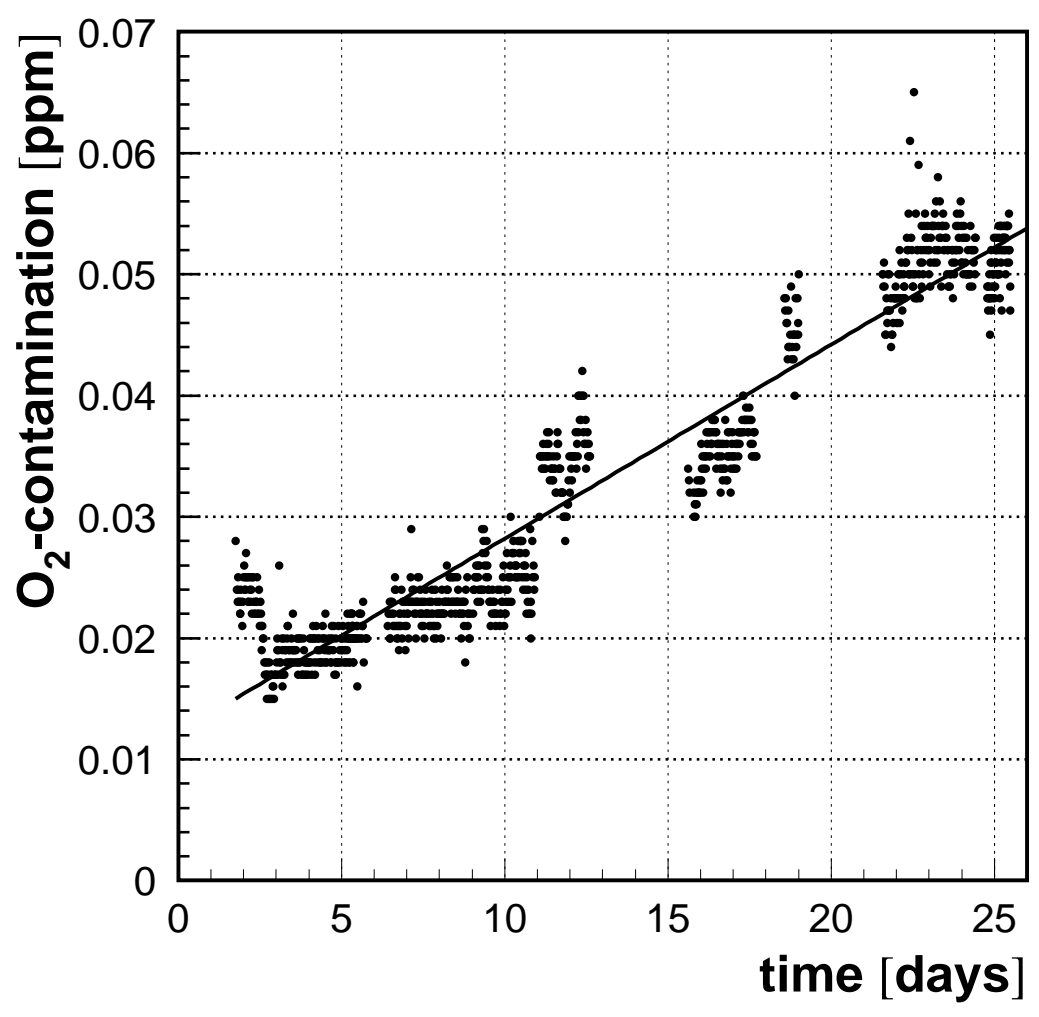

Figure 10: $\mathrm{O}_{2}$-contamination estimated from the signal ratio of the ${ }^{241} \mathrm{Am}$ cell and ${ }^{207} \mathrm{Bi}$ cell. An increase of $1.6 \mathrm{ppb}$ per day has been observed during the run period. 


\subsection{Liquid Argon Temperature Monitoring}

The temperature of the LAr as well as that of the copper absorber plates have been monitored constantly during the cryogenic operation. Platinum PT-100 temperature sensors have been chosen because of the proven long term stability and low sensitivity to magnetic fields. In addition, radiation tests with neutrons and photons have shown that the sensors withstand high radiation levels and still offer a high sensitivity in the temperature range of interest, i.e. 70 to $90 \mathrm{~K}$. The small geometrical size as well as the low price are also advantageous. A special calibration procedure of the sensors has been carried out to achieve a precision of typically 6 to $8 \mathrm{mK}$ during operation.

There are two separate sets of temperature sensors: one set monitors the temperature of the argon and a second set monitors the temperature of the modules.

The set that monitors the argon is made up of eight regular PT-100 probes as well as of two high precision probes. The regular probes are mounted in copper blocks and positioned at different heights inside a tube of $1600 \mathrm{~mm}$ length running vertically inside the cryostat [26]. Each high precision probe is placed next to a regular probe and is used as a reference system. All these probes allow for the control of the process of warming up and cooling down as well as the monitoring of the temperature gradient in the liquid argon.

Six additional probes have been installed at the rear plates of individual modules, as foreseen in the final HEC wheel assembly. These probes monitor the module temperature in particular during the cool down and warm up operations.

A precise resistive direct current bridge is used for the measurement. Two different systems have been used for the signal readout: a 16 bit digital to analog converter (DAC) in combination with a microprocessor (DUBNA-4 system) and a local monitoring box (LMB) developed at CERN as part of the general detector control system (DCS) used in LHC experiments. Both measurement systems gave fully consistent results.

Figure 11 shows the time dependence of the LAr temperature as measured by the various sensors positioned either at different height in a tube inside the cryostat $(\mathrm{S})$ or at the rear plates of individual modules $(\mathrm{P})$ over a time period of 12 hours. The temperature stability of the LAr over a long period of time was typically in the range of 20 to $30 \mathrm{mK}$, except for short periods corresponding to the refilling of the phase separator dewar. For example Figure 12 shows the distribution of the temperature measurements as obtained from a single probe $(\mathrm{S} 1)$ during a 12 hour period. In this case the stability reached is about $2.5 \mathrm{mK}$ rms. 


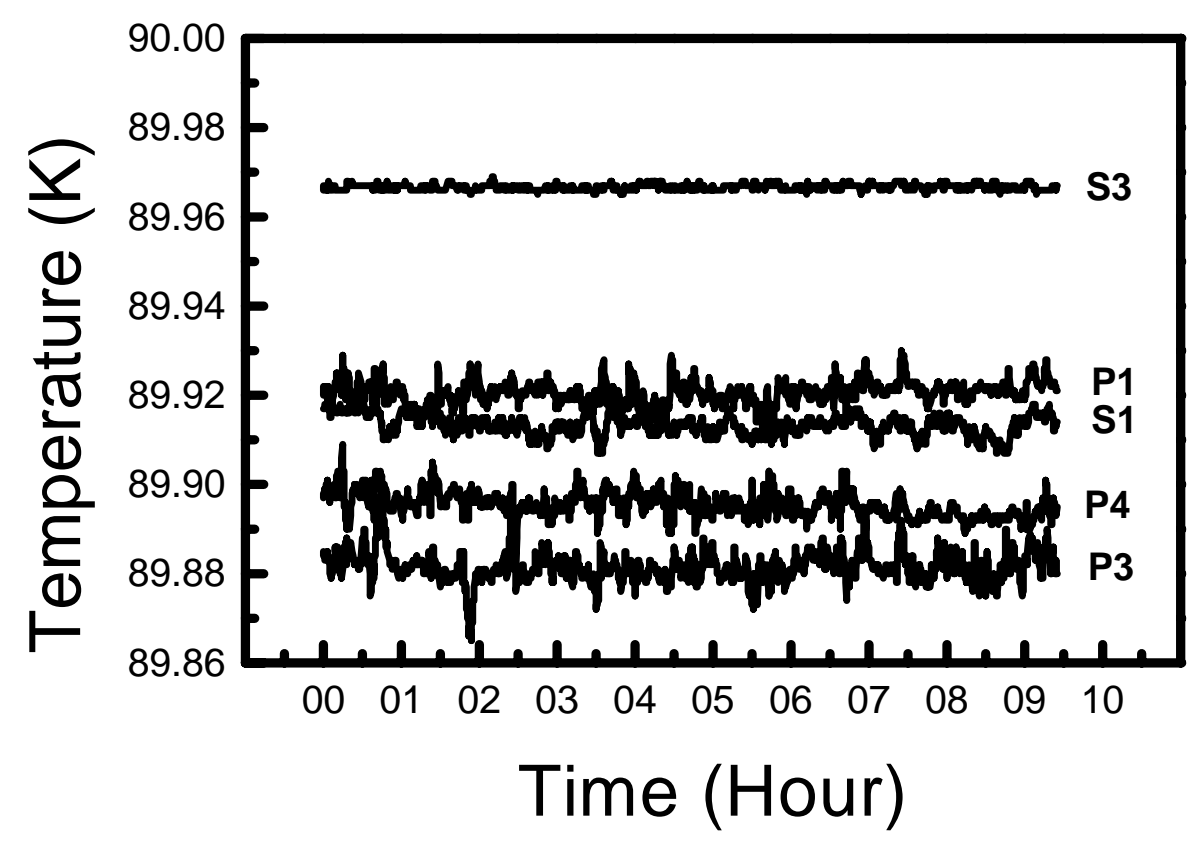

Figure 11: Time dependence of the liquid argon temperature as measured by five sensors positioned either in a tube at different height $(\mathrm{S})$ or at the rear plates of individual modules $(\mathrm{P})$.

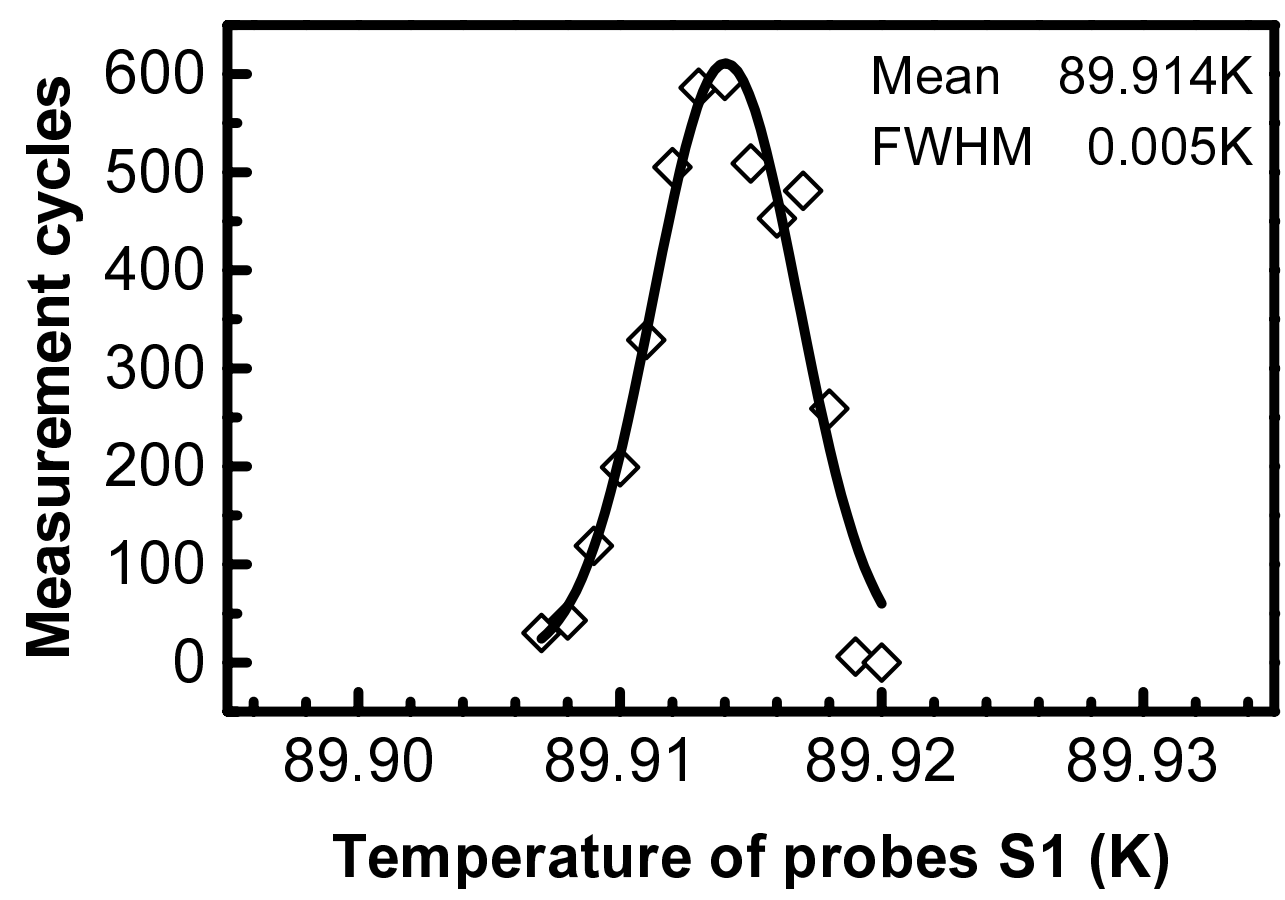

Figure 12: Distribution of the liquid argon temperature measurements as obtained from a single probe (S1) during a 12 hour operation period. 


\section{Data Analysis and Simulation}

\subsection{Data Analysis and Signal Processing}

About 1000 runs with electron, pion or muon data have been taken in each run period. Energy scans in the range $6 \mathrm{GeV}<E<200 \mathrm{GeV}$ have been performed for electrons and pions at 15 different impact points covering the area accessible through the beam window of the cryostat. Within this region the amount of dead material in front of the calorimeter setup is minimal. Figure 13 shows the beam impact points (labeled as A-O) on the front face of the three HEC $\phi$-wedges. These impact points have been selected using simulations of electron data. Their distance to the tie rods (open circles) is large enough to keep signal losses at an acceptable level. In addition, horizontal and vertical scans have been performed for all particle types and at various energies.

For the nominal high voltage $(1800 \mathrm{~V})$ the electron drift time for the HEC LAr gap is $450 \mathrm{~ns}$, so the full signal covers 21 or 22 time samples each separated by $25 \mathrm{~ns}$. For each event trigger, 16 time samples have been read out. In specific runs, e.g. scans in the area near the edge of modules, data taken at lower HV settings or studies for the analysis of the full signal shape, an even larger number of time samples (up to 96) has been used. The signal amplitude has been reconstructed using the optimal filtering technique [22] and the calibration constants. Out of the 16 time samples, five (from the $7^{\text {th }}$ to the $11^{\text {th }}$ ) have been used for this reconstruction. The precision of the signal amplitude reconstruction is typically $0.5 \%$. An important aspect is the quality of the signal waveform description. For a typical read-out channel, Figure 14 shows the reconstructed normalized waveform for electron data. For each event the signal in each time sample is normalized using the reconstructed amplitude. The maximum is expected to be at 1.0 for a perfect signal waveform reconstruction. The observed deviation is about $\pm 0.5 \%$ reflecting the accuracy of the procedure applied. This precision depends critically on the parameterization of the particle signal as predicted from calibration data. The first five time samples preceding the event pulse are used to define the pedestal value and the noise (again using the optimal filtering technique) of each individual read-out channel. The noise suppression factor from optimal filtering is typically $\sigma_{\text {noise }}^{\mathrm{OF}} / \sigma_{\text {noise }} \approx 0.6-0.7$, where $\sigma_{\text {noise }}$ refers to the spread of the electronic noise seen in an individual time sample. The ADC clock is not synchronized with the particle trigger, therefore the start of the signal is randomly distributed within a range of $25 \mathrm{~ns}$ with respect to the clock signal. The timing information is supplied by the TDC module and used to predict the signal position within the ADC window. The dependence of the optimal filtering weights on the TDC data is obtained from the analysis of calibration data. The time dependence of each weight is parameterized by a $4^{\text {th }}$-order polynomial. The parameterized functions are used to calculate the weights for each event.

Due to thicker copper absorber plates, the HEC2 wheel has a sampling ratio which is smaller by a factor of two with respect to the HEC1 wheel. Therefore the visible energy $E_{\mathrm{nA}}^{\mathrm{vis}}$ for HEC2 has been multiplied by a factor of two prior to extracting any calibration constant. For the longitudinal segment with one HV line disconnected a geometrical correction factor of $4 / 3$ has also been applied.

To reconstruct the energy, clusters of cells have been defined in each longitudinal segment. The cluster size has been kept fixed for each impact point so that the noise 
contribution can be better studied and controlled. Read-out channels near each impact point were included if they had an average signal of more than $200 \mathrm{nA}$ for electrons at $175 \mathrm{GeV}$ or $15 \mathrm{nA}$ for pions at $180 \mathrm{GeV}$. This selection yields a typical cluster size of 6 read-out channels for electrons and about 50 to 60 read-out channels for pions. Rather large cluster sizes have been chosen in order to keep losses due to energy leakage small. Simulation studies give an estimated energy leakage of $\sim 0.4 \%$ for electrons and $\sim 5 \%$ for pions. For pions this is dominated by energy leakage outside the three HEC $\phi$-wedges, which is typically at the level of $\sim 3.5 \%$. To estimate the electronic noise contribution, the noise from the cluster channels has been summed. Thus any correlation between different channels or any coherent part in the noise is automatically taken into account. This holds since the noise in the calibration data, which are used to extract the optimal filtering weights, is similar to the noise in the particle data. Given these cluster sizes, the equivalent noise amounts to $\sim 0.6 \mathrm{GeV}$ for electrons and $\sim 6 \mathrm{GeV}$ for pions.

All data have been processed using the standard HEC offline reconstruction program [13], which comprises pedestal and signal amplitude reconstruction as well as trigger handling, cluster reconstruction and slow control data monitoring. 


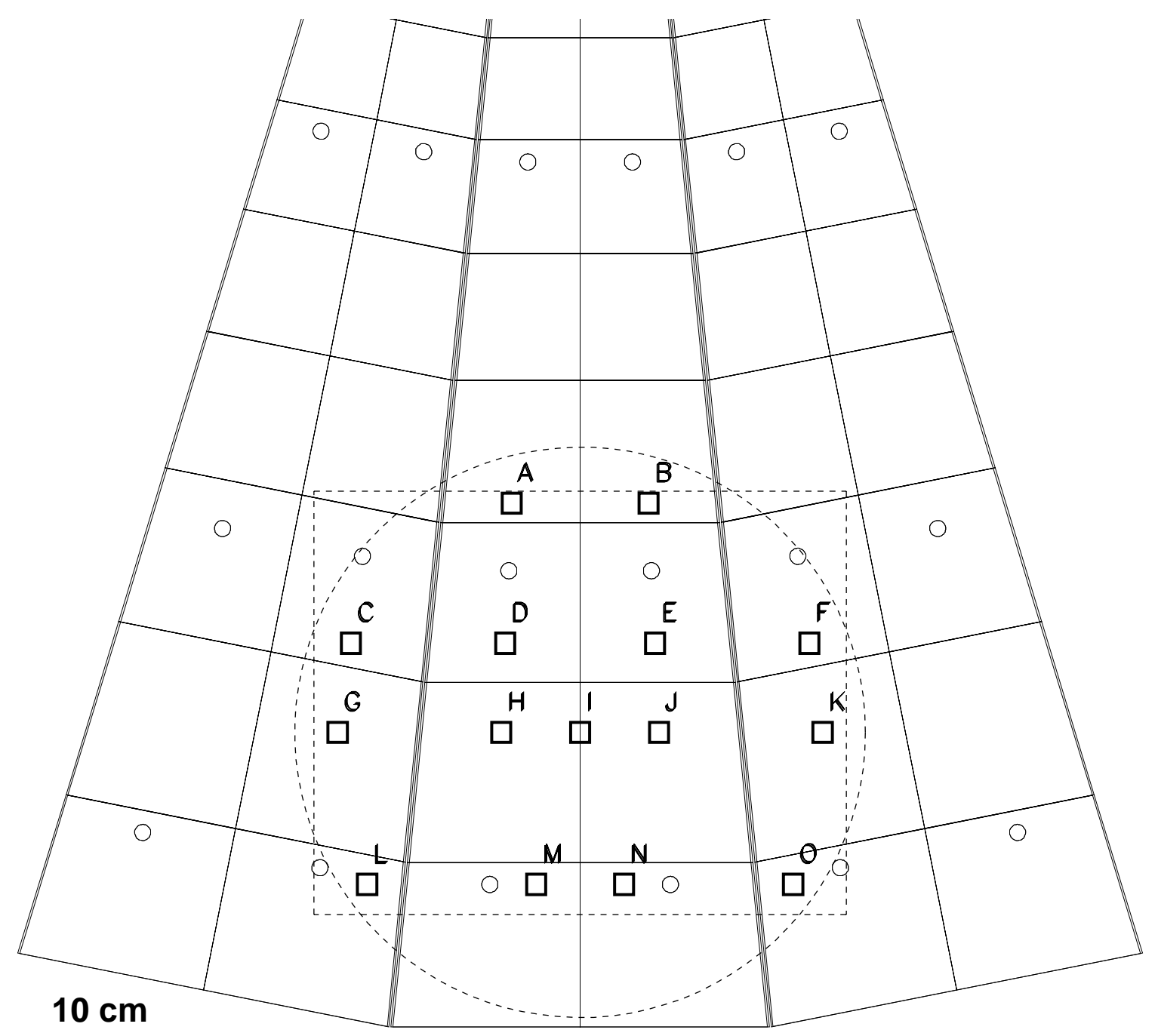

Figure 13: The beam impact points (open squares) on the front face of the calorimeter setup with three $\phi$-wedges. Data taken at these points have been used for the energy scans. The open circles locate the tie rods. The dashed circle represents the area accessible through the cryostat window, while the dashed rectangle represents the area accessible through beam bending (vertical) and cryostat motion (horizontal). 


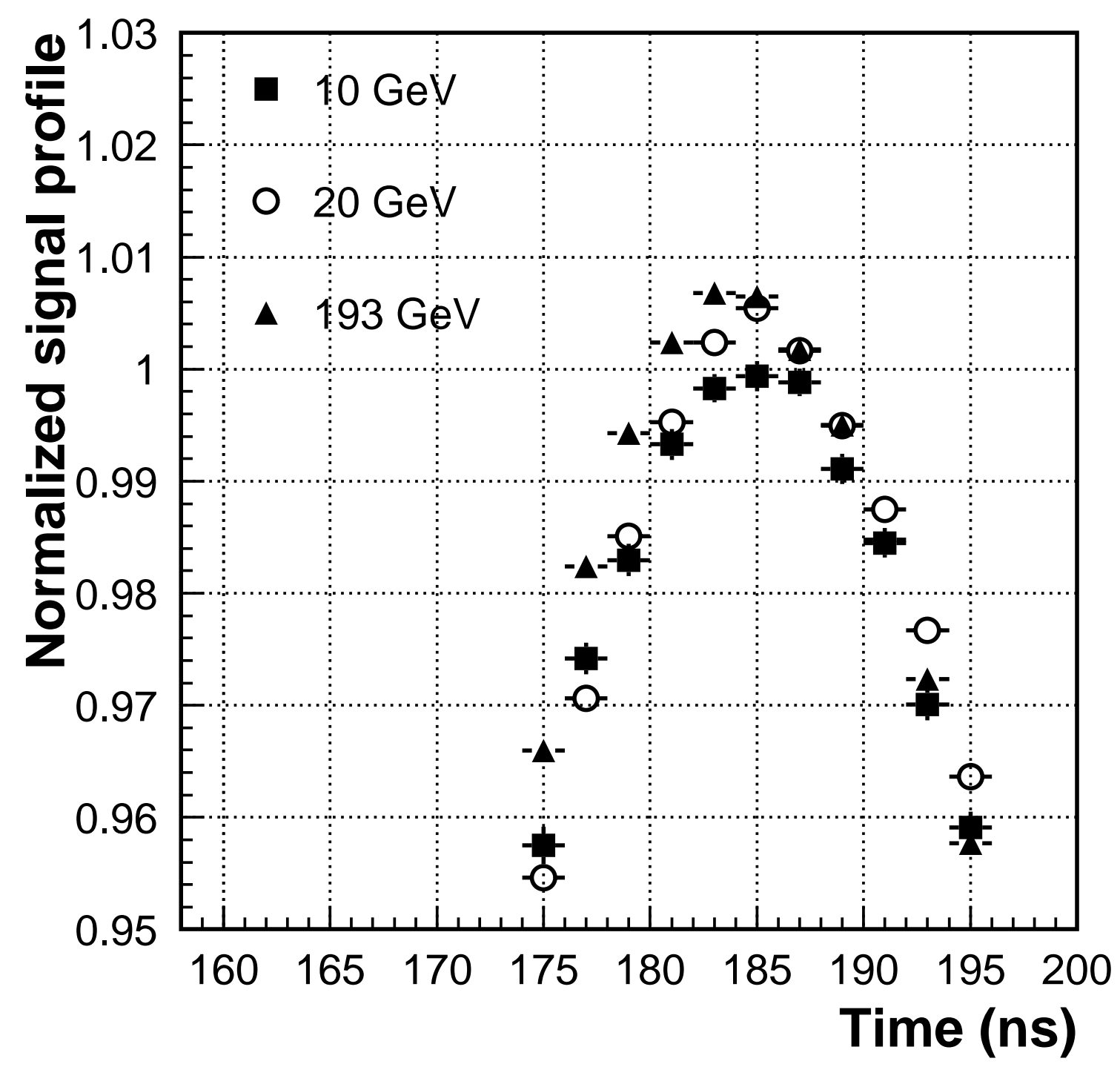

Figure 14: Reconstructed normalized signal waveform for electrons of different energies. 


\subsection{Monte Carlo Simulation}

The evaluation of the HEC performance requires the comparison of experimental data, obtained at beam tests, with detailed Monte Carlo simulations. To fulfil this task a special software package has been prepared [27]. It allows for the simulation of the response of the HEC modules to various particle beams of different energies, available at CERN beam tests. This package uses the standard ATLAS software [28] (DICE 3, ATLSIM) with GEANT 3.21 [29] for the detector response simulation.

The geometry of the beam test setup is described in full detail. For example, the HEC module description includes not only copper plates and gaps of liquid argon, but also polyimide electrodes, copper pads and tie-rods. All H6 beam elements, such as the cryostat, multiwire proportional chambers and scintillating counters, extended over a distance of 30 meters, are included as well. Three different codes for the hadronic shower development, GFLUKA, GCALOR and GHEISHA, are available for simulations.

Prior to the analysis of simulated events, hardware conditions (e.g. reduced signal in individual gaps due to disconnected HV lines) have been taken into account. For the electron and pion beam test data the actual noise has been subtracted. For muon simulation the actual electronic noise has been added to the signal using beam test events. For each read-out channel and each individual event the noise has been added to the simulation with a scale factor $\kappa=\alpha_{e m} / \alpha_{e m}^{M C}$, transforming the noise from the nA scale to the $\mathrm{GeV}$ scale of visible energy. Here $\alpha_{e m}^{M C}$ is a dimensionless ratio $E_{\text {nominal }}^{\text {beam }} /<$ $E_{v i s}^{\text {total }}>=23.30 \pm 0.01$, found using electron MC samples. The difference in sampling ratios between the HEC1 and HEC2 wheel has been included. The leakage of energy of electromagnetic showers out of a selected cluster (see Figure 15) has been taken into account in the determination of the scale factor $\kappa$. All the procedures and program chains, which were used to analyse Monte Carlo events (after hardware corrections described above), are exactly the same as those used for the beam test data.

The layout of the HEC beam test setup with only three $\phi$-wedges and the absence of an electromagnetic calorimeter in front, causes a problem with respect to the longitudinal and lateral leakage of energy for hadronic showers. To estimate the influence of the energy leakage on the performance of the calorimeter, "virtual" leakage detectors are implemented. In these leakage detectors the kinetic energy is summed up for all particles leaving the HEC modules through lateral and back sides.

Figure 16 shows the energy dependence of the total energy leakage as well as lateral and longitudinal ones as expected from Monte Carlo simulations for pions at a central impact point. For all energies the fraction of energy lost is typically at the level of 3 to $3.5 \%$. At low pion energies the losses are dominated by the lateral leakage due to the limitation to three $\phi$-wedges. At high energies the longitudinal leakage starts to play a role as well. The leakage of energy of hadronic showers out of the selected cluster (see Figure 17) is usually smaller than the leakage outside the HEC modules. 


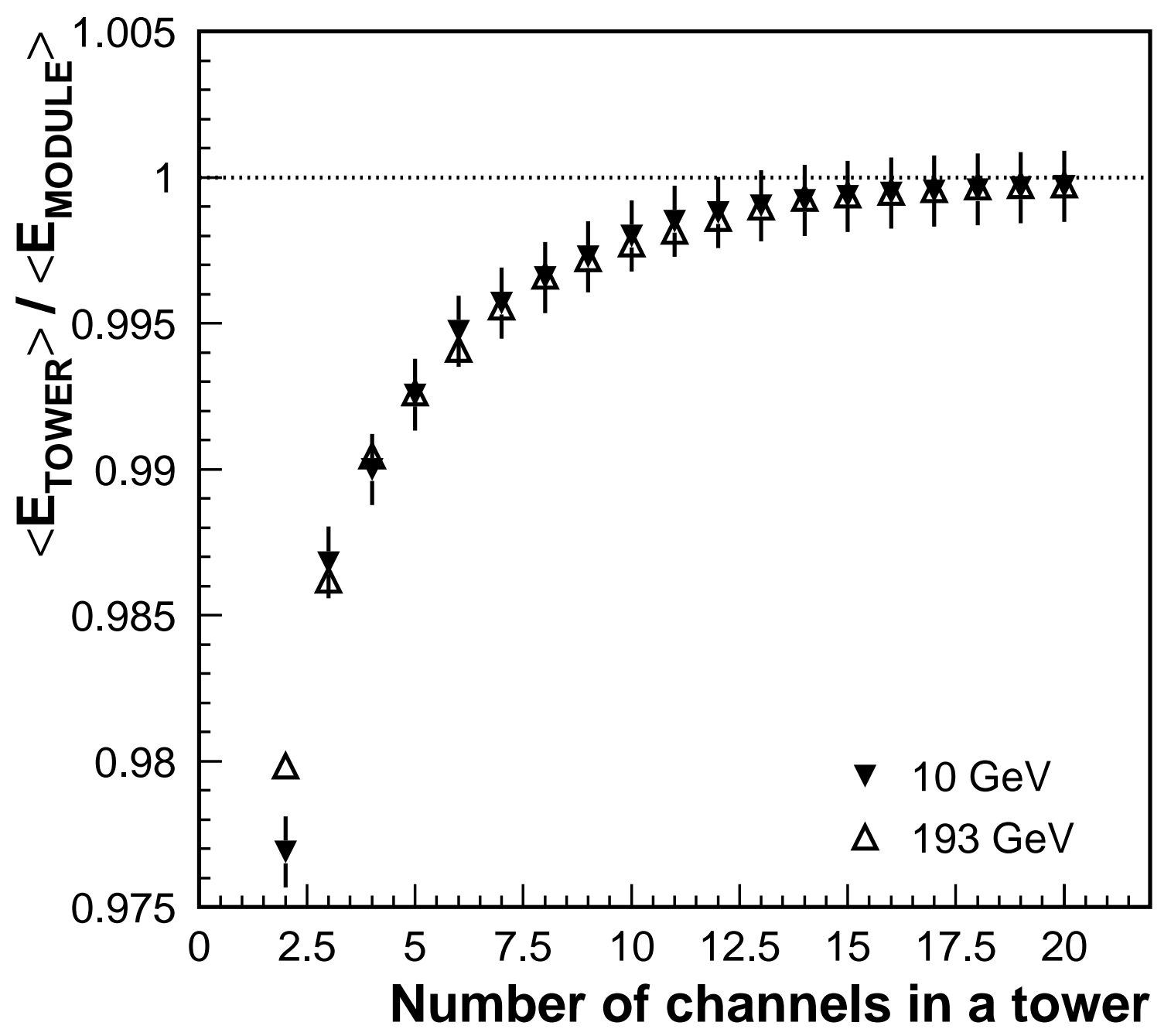

Figure 15: The relative amount of the energy in a cluster with respect to the total visible energy in HEC modules for electrons as a function of the number of read-out channels used in the cluster. 


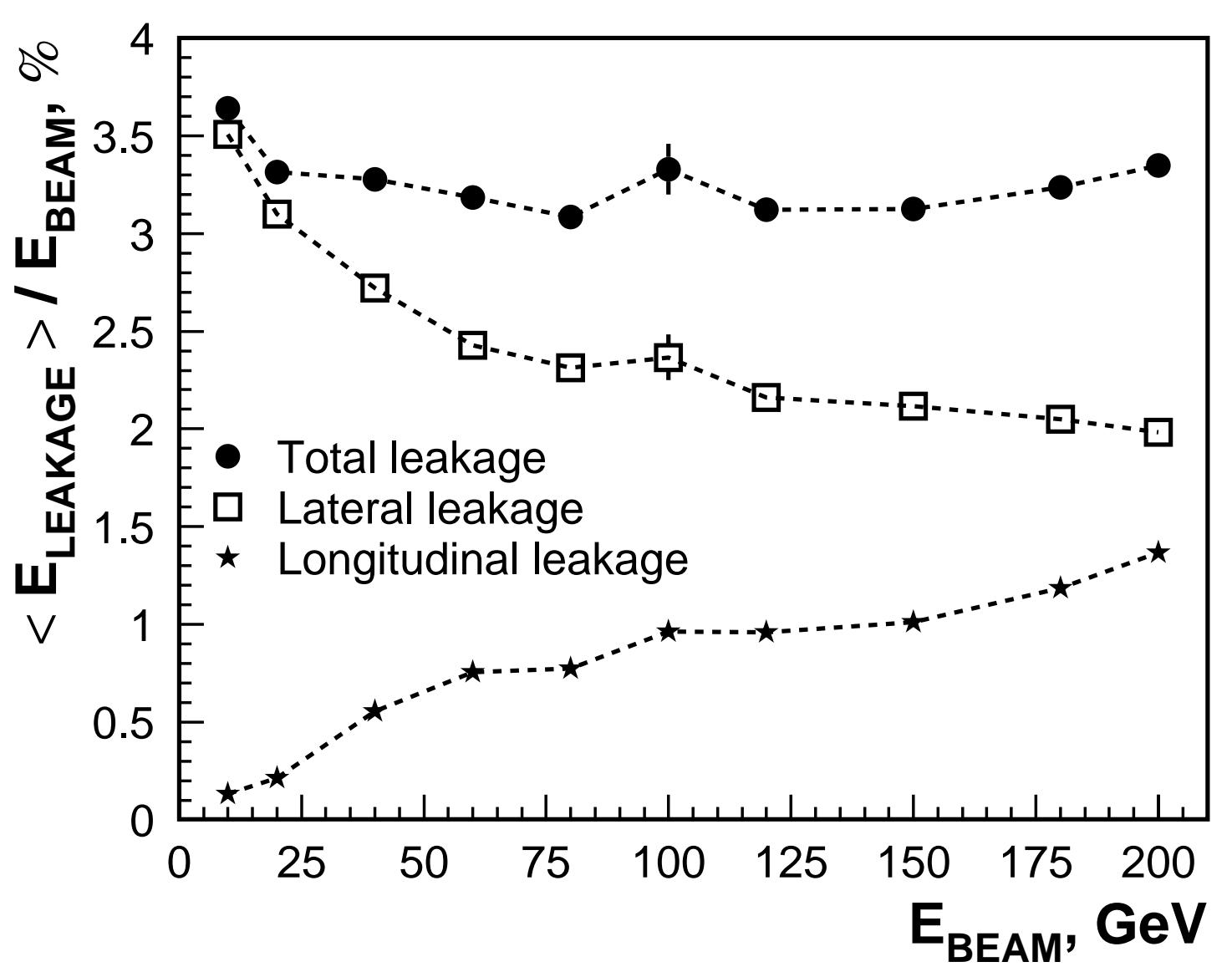

Figure 16: The relative amount of the leakage energy with respect to the pion beam energy as a function of the beam energy. 


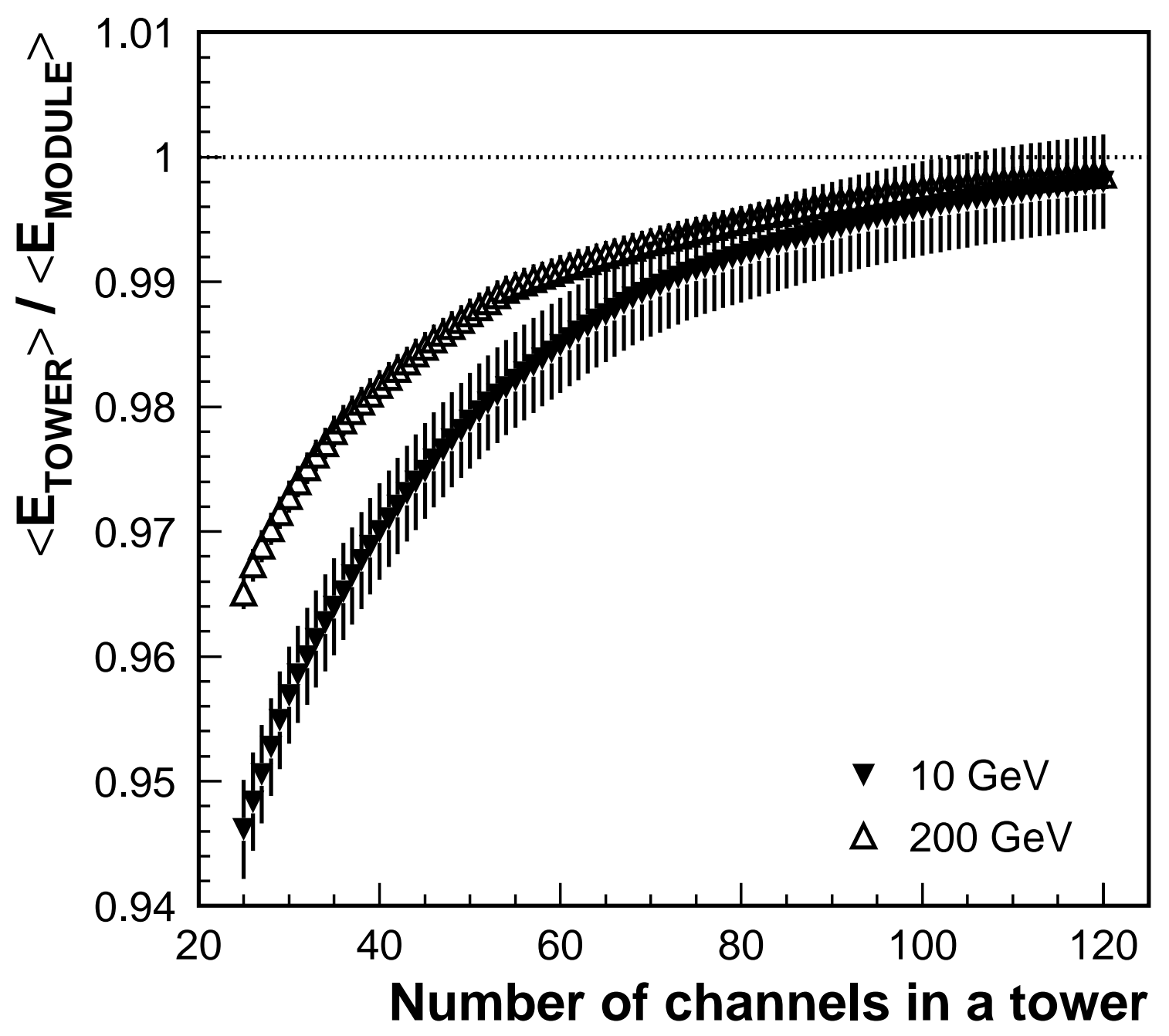

Figure 17: The relative amount of the energy in a cluster with respect to the total visible energy in all HEC modules for charged pions as a function of the number of readout channels used in the cluster. 

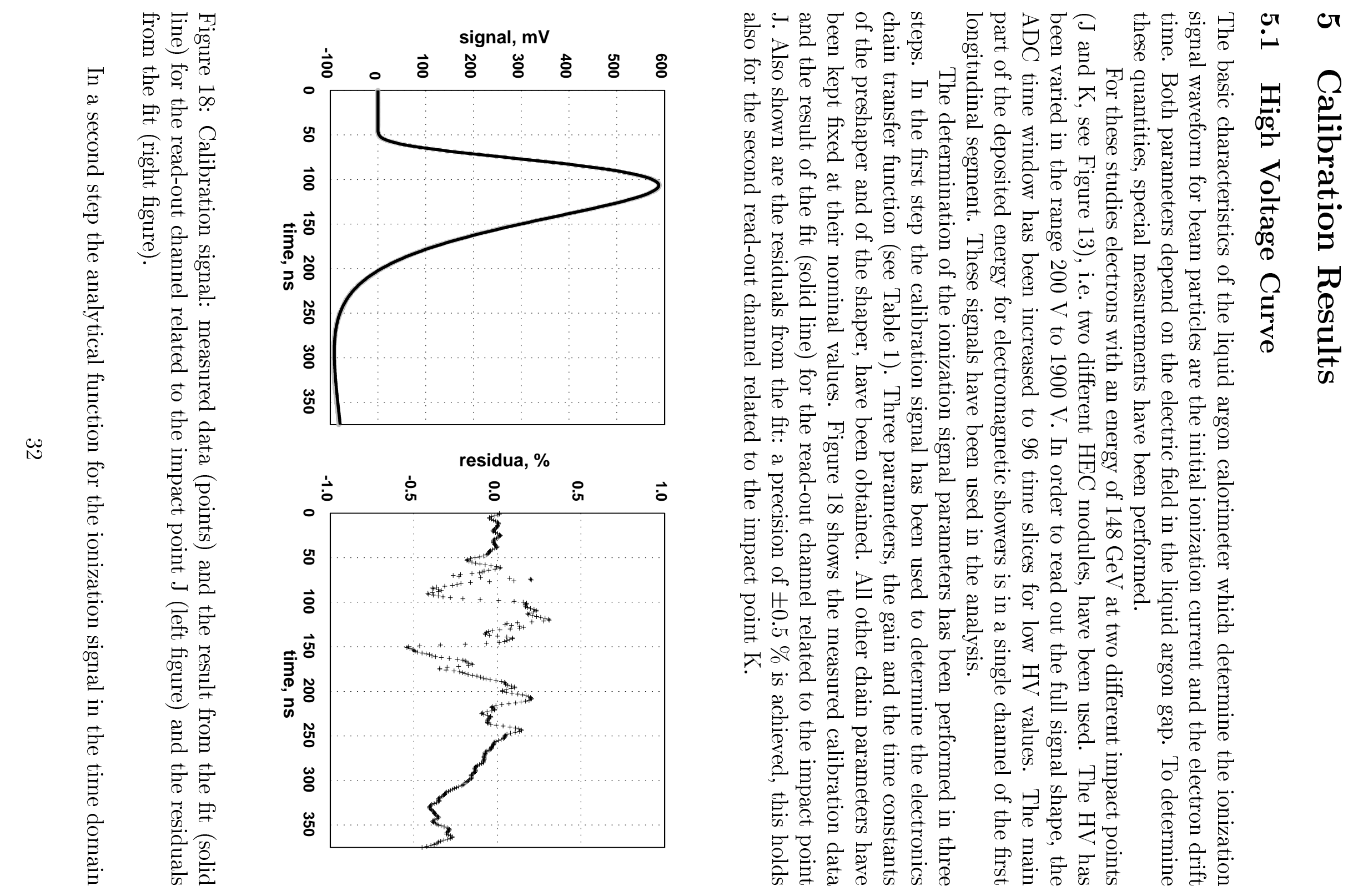
has been derived. Finally this function has been applied to the measured signals to determine the initial ionization current and the electron drift time $\tau_{d r}$. The measured and predicted ionization signals at two HV settings for the read-out channel related to the impact point $J$ are shown in Figure 19. The precision of the signal shape prediction is within $\pm 1 \%$ for both channels and for all HV settings.

The drift velocity and electric field have been calculated using the nominal value of the liquid argon subgap thickness of $1.97 \mathrm{~mm}$. Figure 20 shows the drift velocity determined for the impact point J (circles) and point K (squares). During these measurements the variation of the argon temperature was in the range $89.45 \mathrm{~K}$ to $89.65 \mathrm{~K}$. The solid lines in Figure 20 present the calculated drift velocity for the median temperature of $89.55 \mathrm{~K}$ using the parameterization [30]. Our data seem to indicate a somewhat longer drift time, the difference being typically $1 \%$ to $2 \%$. The initial ionization current depends on the electric field due to two reasons: for an increase in the electric field, (i) the drift velocity increases, and (ii) the primary recombination decreases. The second effect can be described by the Thomas-Imel model [31] as follows:

$$
Q_{P}=Q_{O} \frac{\ln (1+C / E)}{C / E}
$$

where $Q_{P}$ is the ionization charge released, $Q_{O}$ is the ionization charge in the case of an infinite electric field (no recombination), and $C=0.84 \mathrm{kV} / \mathrm{cm}$ is a constant. The ionization current is calculated from the charge using the measured drift time. Figure 21 shows the data as well as the fit of the data to the Thomas-Imel model as given by equation 2.

From the value of $Q_{O}$ and the ionization energy per electron-ion pair of $23.2 \mathrm{eV}$ (see [32] and references therein) the energy deposited in the liquid argon gap can be calculated. The values of $5.53 \mathrm{GeV}$ for the impact point $\mathrm{J}$ and $5.60 \mathrm{GeV}$ for the impact point $\mathrm{K}$ have been obtained. The corresponding values obtained from simulation using GEANT 3 are $5.53 \mathrm{GeV}$ and $5.58 \mathrm{GeV}$ respectively. The systematic error on this prediction is estimated to be $+1 \%$ due to different energy cuts in GEANT 3 and $-2 \%$ due to the passive volume of the honeycomb spacer mats which are not included in the simulation. The agreement with data is within errors. 

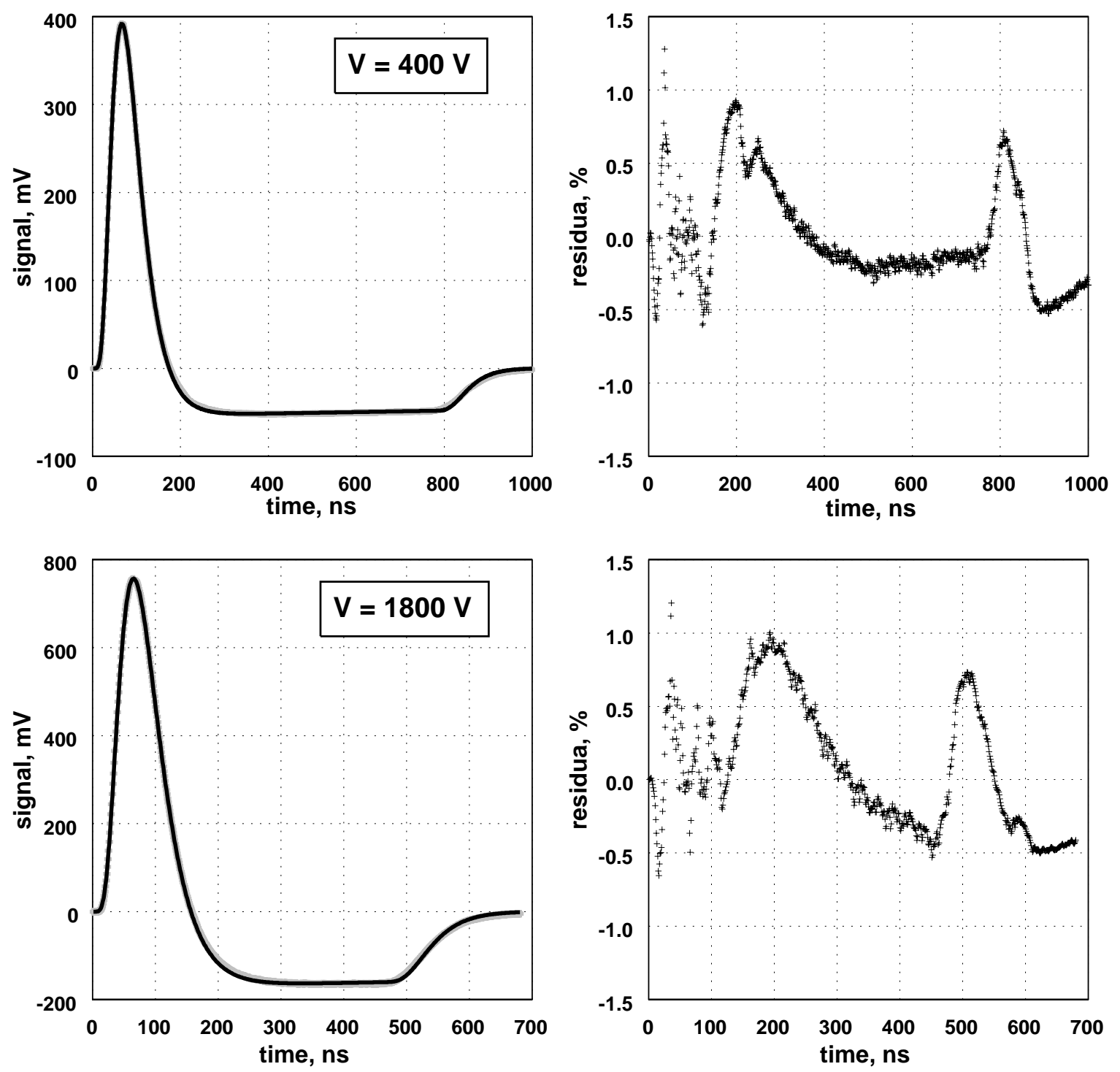

Figure 19: Ionization signal from electrons. Shown are the data for two different HV settings for the read-out channel related to the impact point J (left figures). Also shown are the predicted signals (solid line) and the related residuals (right figures). 


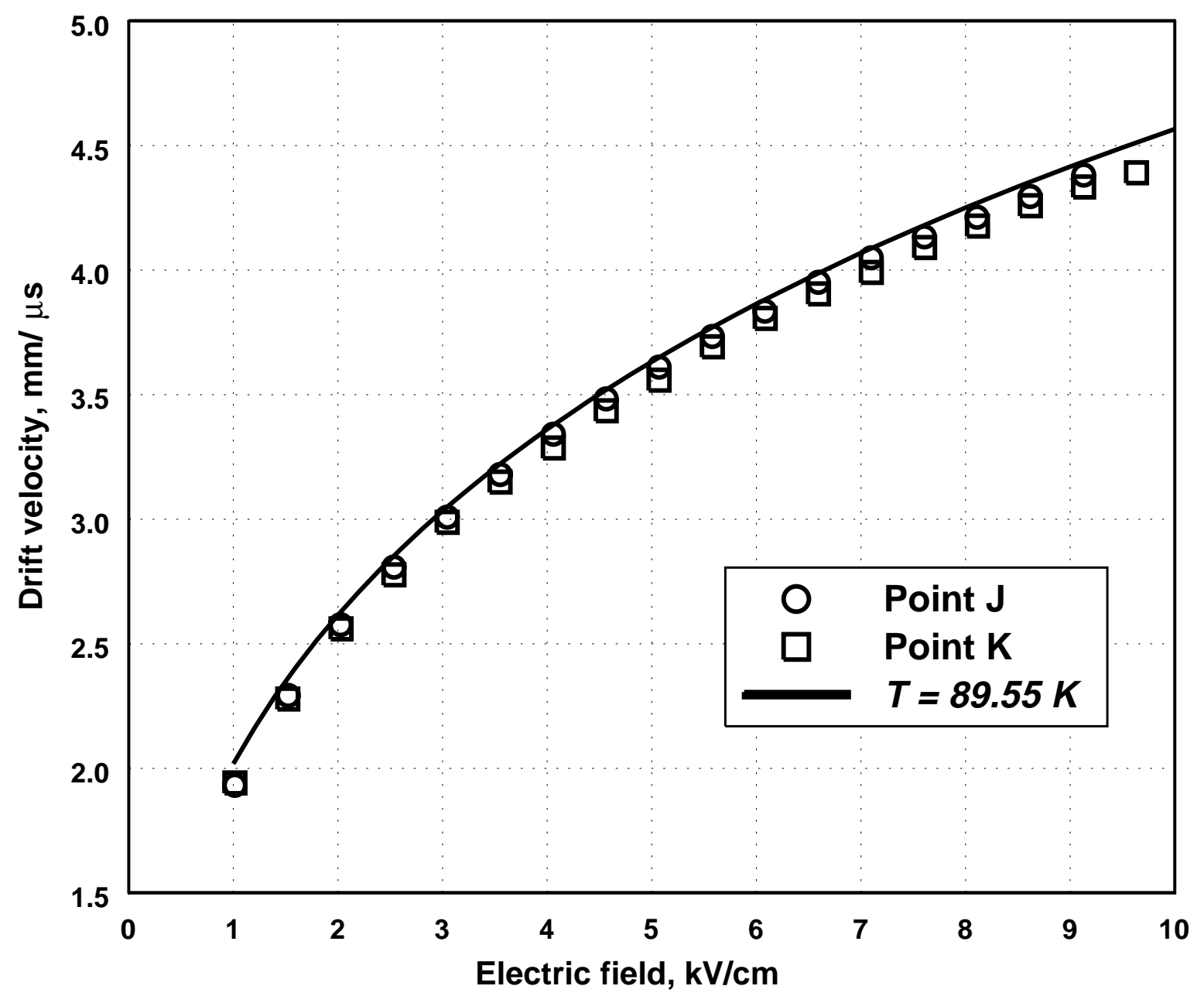

Figure 20: The dependence of the electron drift velocity on the electric field. Shown are the data for the read-out channels related to two different impact point (dots and squares). The solid line represents recently published data (see text for reference). 

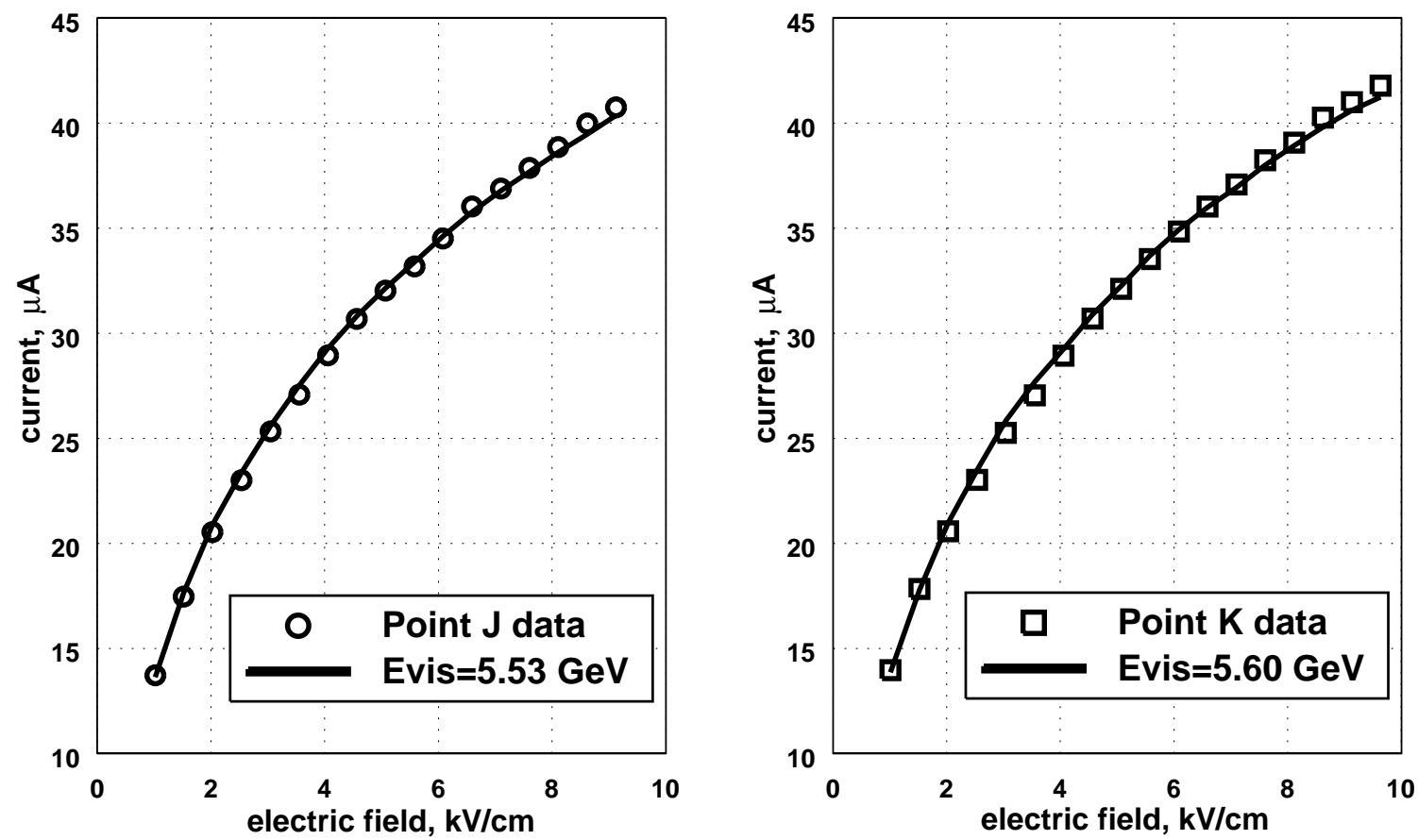

Figure 21: The initial ionization current (points) and the fit to the Thomas-Imel model (solid lines) for the two impact points. 


\subsection{Response to Electrons}

The deposited energy, as reconstructed from the cluster, is obtained in units of nA and has to be converted to the related particle energy. To do so, an average scale factor $\alpha_{e m}=$ $2.93 \pm 0.03 \mathrm{MeV} / \mathrm{nA}$ has been obtained for the data. This electromagnetic calibration constant has been applied to the electron data for all energies. Figure 22 shows the energy distribution of electrons with an energy of $10,20,80$ and $147.8 \mathrm{GeV}$ at a particular impact point. The solid lines represent gaussian fits to the distributions within a region of $\pm 2 \sigma$ around the mean value.

Figure 23 shows the energy dependence of the energy resolution $\sigma / E$ for electrons at three different beam particle impact points. The electronic noise in the related electron cluster has been obtained as described above and it has been subtracted quadratically for each individual energy and impact point. Thus any time or impact point dependence of the electronic noise has been taken into account, as well as the contribution due to coherent noise effects (see discussion above). The energy dependence of the energy resolution has been parameterized by

$$
\frac{\sigma(E)}{E}=\frac{a}{\sqrt{E}} \oplus b
$$

with $a$ being the sampling term and $b$ the constant term. The lines show the result of the fit to these data points using the ansatz given above.

The results for the sampling term $a$ and the constant term $b$ obtained for the various beam particle impact points are shown in Figure 24. The average values obtained are $a=$ $(21.4 \pm 0.2) \% \mathrm{GeV}^{1 / 2}$ and $b=(0.3 \pm 0.2) \%$, to be compared with simulation expectation of $a=(21.7 \pm 0.1) \% \mathrm{GeV}^{1 / 2}$ and $b=(0.0 \pm 0.2) \%$. The data as well as the expectation (solid line, the error indicated by the dashed lines) are shown. The agreement is rather good. An important aspect of the calorimeter performance is the spatial uniformity of the response. This aspect can be tested by comparing data from different beam impact points. Figure 25 shows the the relative variation of the electromagnetic calibration constant $\alpha_{e m}$ with the beam particle impact position. The data are in a band of typically $\pm 1 \%$, leaving not much room for any channel intercalibration errors.

Finally the linearity of the signal response has been studied as well. Figure 26 shows the relative variation of the inverse of the electromagnetic calibration constant $\alpha_{e m}$ with beam energy. The circles show the results for the data at four different impact points and the stars the corresponding expectations from simulation. The response is linear within a band of $\pm 1 \%$. The decrease of the response at very low energy is mainly due to the dead material $\left(0.6 X_{0}\right)$ in front of the calorimeter. 

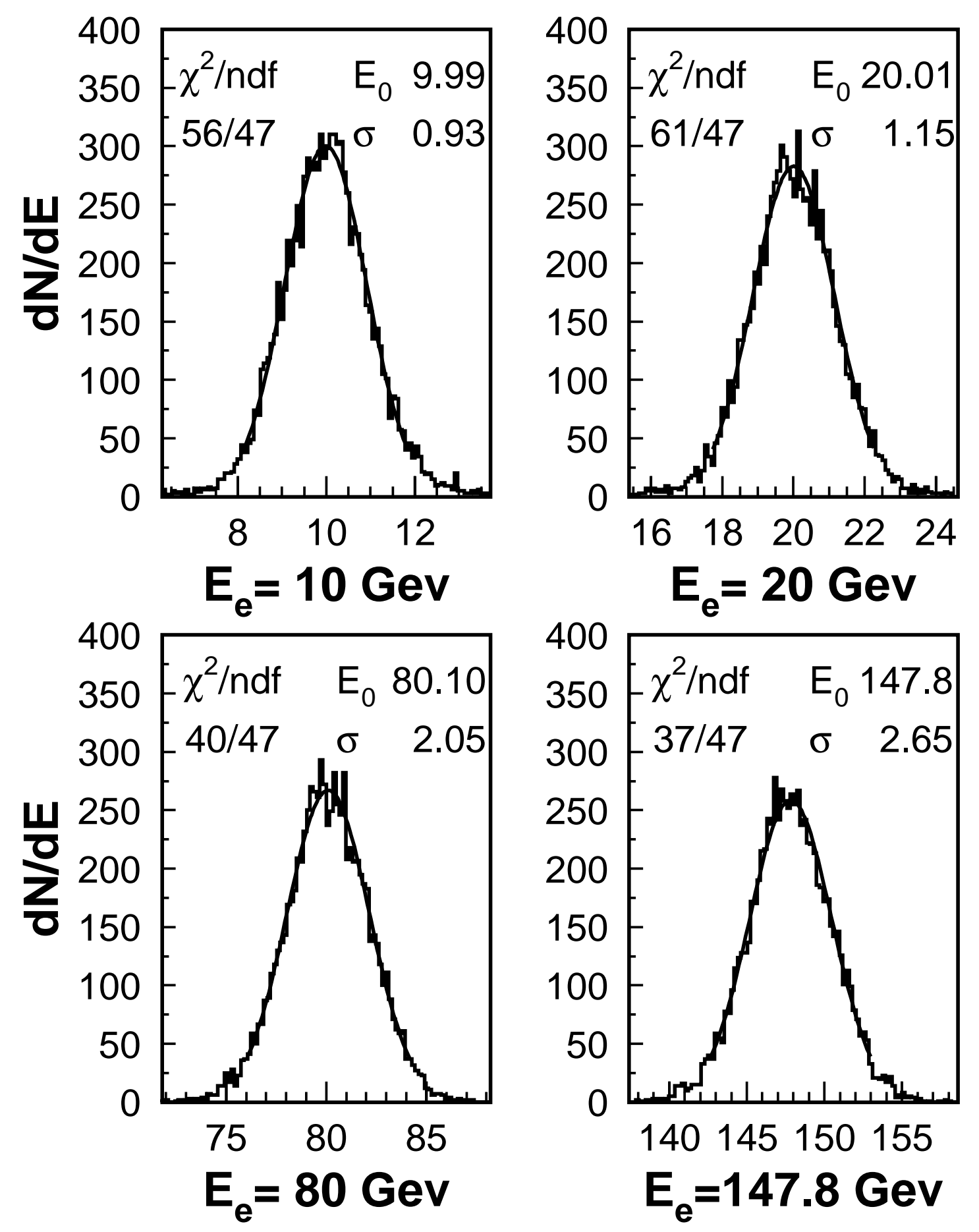

Figure 22: Energy distribution for electrons with an energy of 10, 20, 80 and $147.8 \mathrm{GeV}$. 


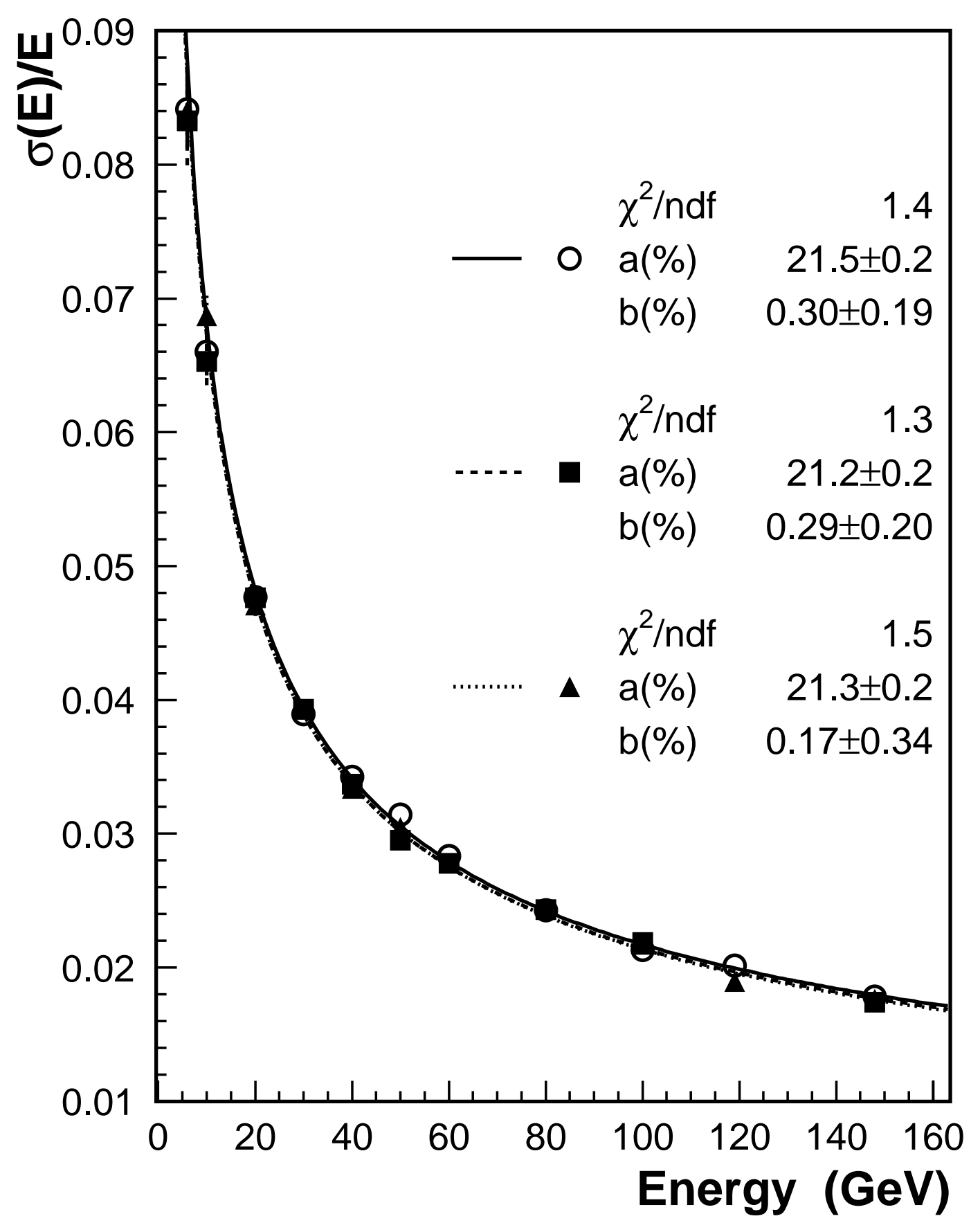

Figure 23: Energy dependence of the energy resolution for electrons at three different beam particle impact points. 

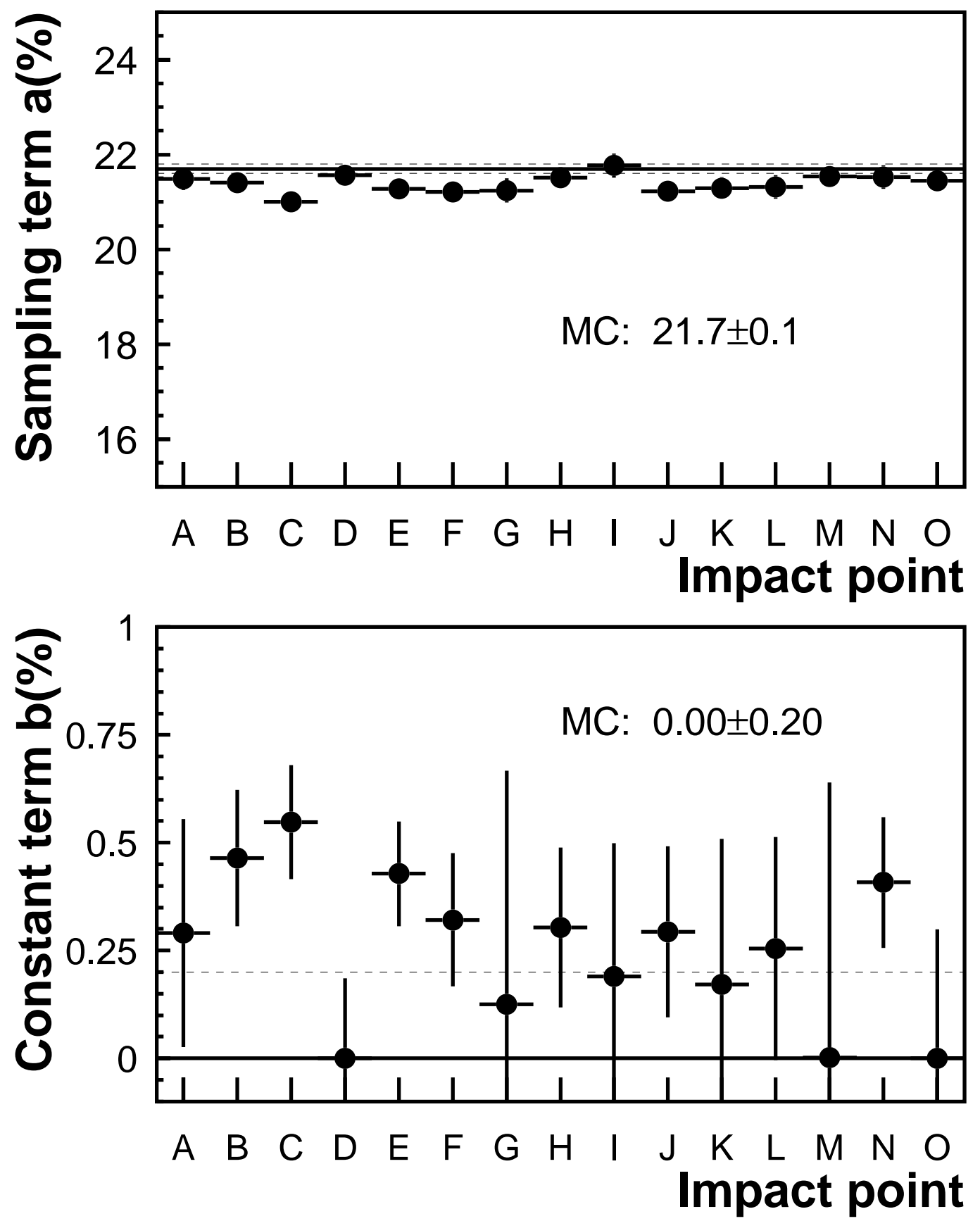

Figure 24: Energy resolution for electrons at different beam particle impact points: shown (circles) are the sampling term $a$ (top) and constant term $b$ (bottom) as obtained from a fit to the beam test data (see text). The horizontal lines and the numbers show the expectation from simulation. 


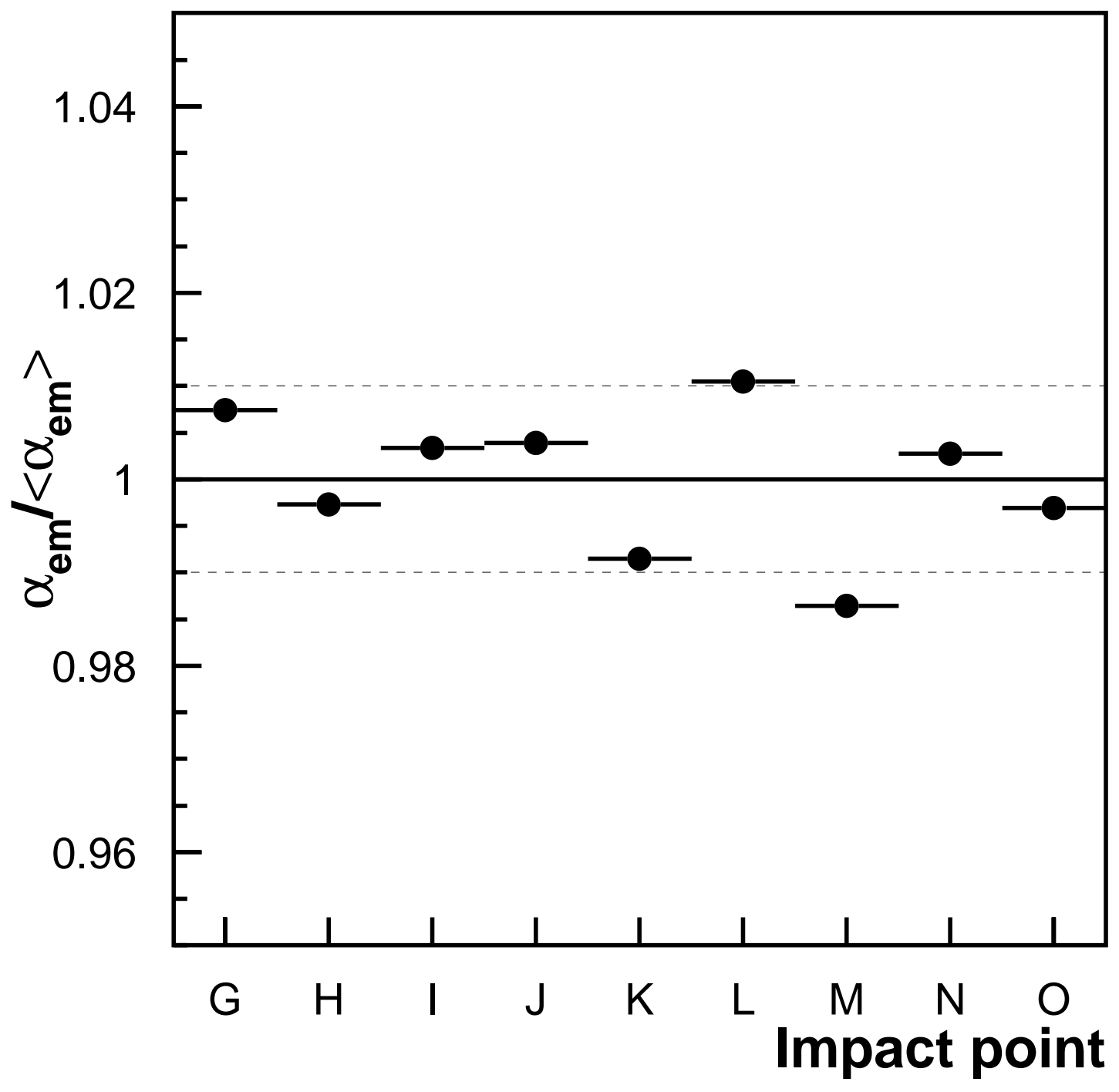

Figure 25: Spatial uniformity of the signal response of electrons: shown is the variation of the electromagnetic calibration constant $\alpha_{e m}$ with the beam particle impact position relative to the average $\alpha_{e m}$. 

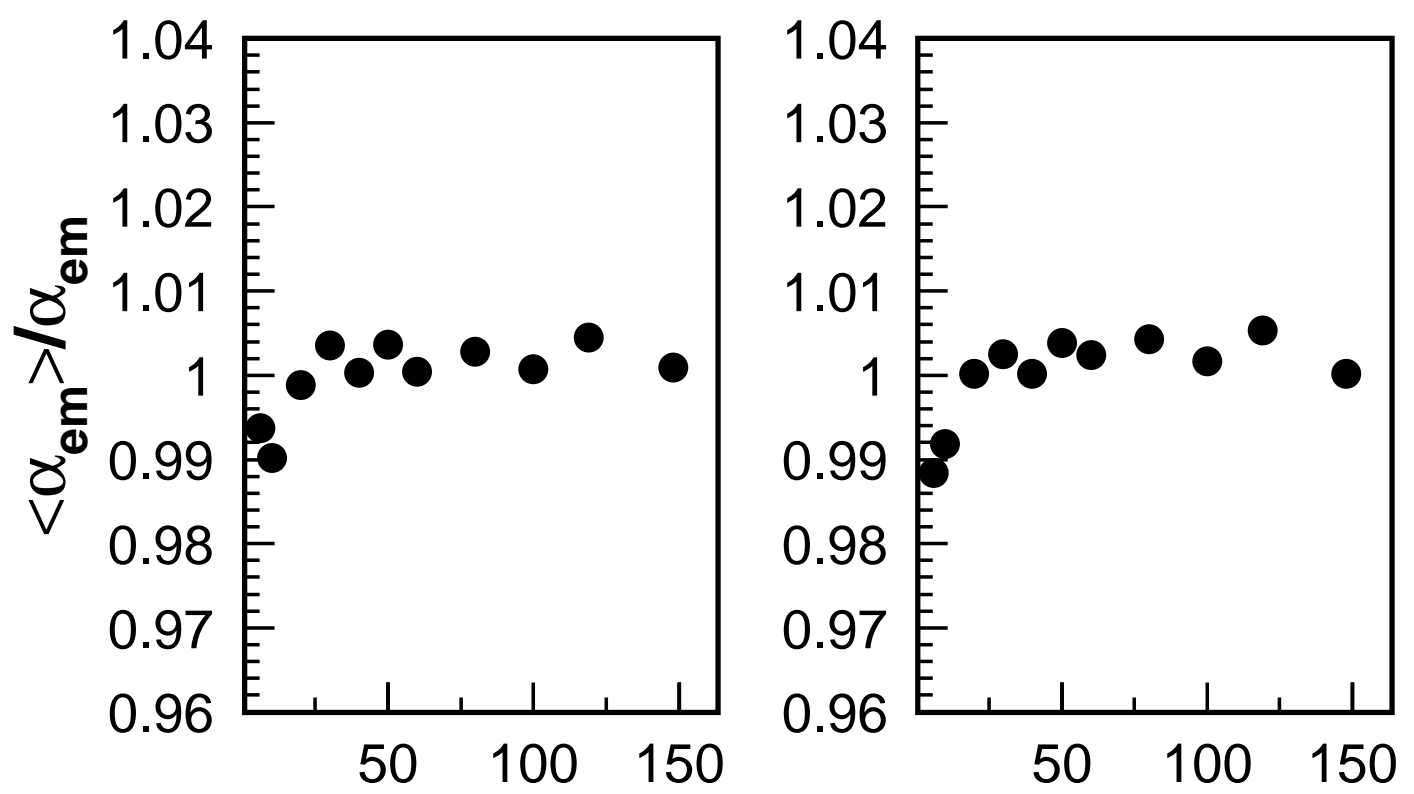

Energy (GeV)
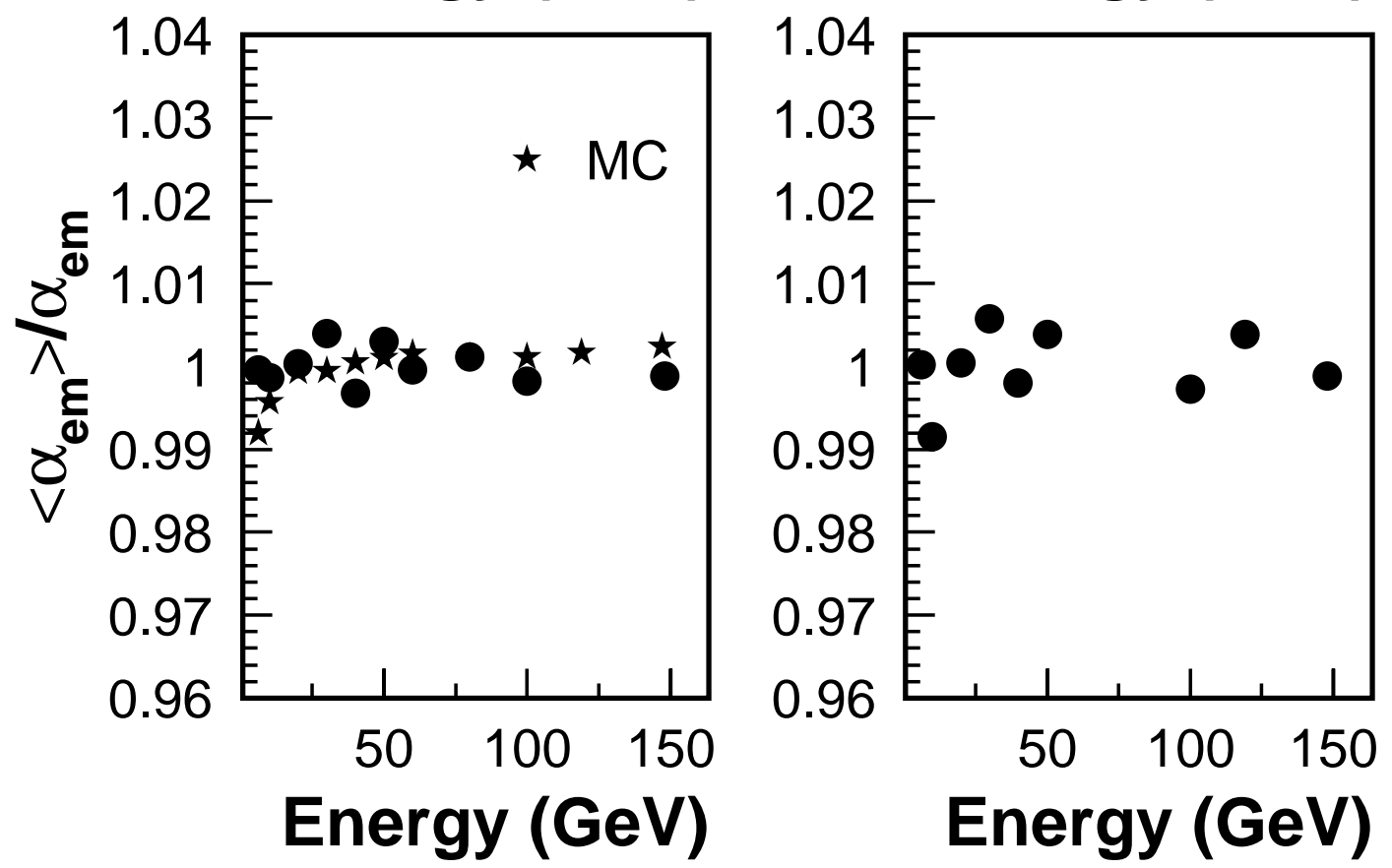

Figure 26: Linearity of the signal response of electrons at four different impact points: shown is the relative variation of the inverse of the electromagnetic calibration constant $\alpha_{e m}$ with beam energy. 


\subsection{Response to Muons}

The muon data are essential to obtain information on the calorimeter response in the region of low deposited energy. In addition, horizontal and vertical scans test the homogeneity of the calorimeter at full depth.

Muon data have been taken at energies of $120 \mathrm{GeV}, 150 \mathrm{GeV}$ and $180 \mathrm{GeV}$ in the two run periods. The MWPC data have been used to reconstruct the beam particle trajectories. The extrapolation of these trajectories has been used to define the readout channels where the muon signal is expected. The impact angle of particles is $90^{\circ}$ at all impact positions. In consequence the read-out structure is, in contrast to the final situation in the ATLAS detector, non "pseudo-pointing" in $\eta$. Therefore the typical number of read-out channels used for the energy reconstruction is typically six instead of four as for the ATLAS setup. Consequently, the noise contribution to the muon signal is increased accordingly. Simulation studies show that the losses of the visible energy due to the reconstruction algorithm are at the level of about $1-2 \%$ only.

Figure 27 shows the comparison of the total energy deposited with the noise contribution for $180 \mathrm{GeV}$ muons. The distributions are plotted in units of the noise width $\sigma_{\text {noise }}$, the ratio of the muon signal to the noise is $\sim 5$. Figure 28 compares the response with expectations for muons of $120 \mathrm{GeV}$. For the simulation the GEANT3 code has been used. The distribution of the data, which is covering more than four orders of magnitude in signal height, is well described by the simulation.

Horizontal and vertical scans have been carried out at various impact points and at all energies. Figure 29 shows the result of the horizontal scan with muons of energy of $180 \mathrm{GeV}$ across the total accessible region, i.e. almost all three $\phi$-wedges of the test beam setup. Shown is the reponse in each individual longitudinal segment. Clearly visible are the positions of the two cracks between the modules. The data reflect also clearly the longitudinal calorimeter structure: eight LAr gaps for the segments one, three and four and 16 LAr gaps for the segment two. The reduced signal in the first longitudinal segment, typically $12 \%$ with respect to the third segment, compares within errors with the expectation of $11 \%$. This reduction of the signal is due to the reduced contribution of the $\gamma$-radiation to the muon signal in the first longitudinal segment. These distributions are shown in more detail in the Figure 30 for the two crack regions between the neighbouring $\phi$-wedges. The lines shown reflect gaussian fits in the crack zones to guide the eye. The dip structure in segment three is somewhat broader, reflecting a larger crack size of HEC2 (segment three only) at this particular height due to additional notches in the copper plates for the signal lines.

Finally the electron to muon ratio has been extracted from the data. All results have been corrected for minor shifts due to noise contributions. For the most probable muon signal we obtain $0.93 \pm 0.02$ for muons of $150 \mathrm{GeV}$ and $0.92 \pm 0.02$ for muons of $180 \mathrm{GeV}$. Theses results have to be compared with the $\mathrm{MC}$ expectations of $0.91 \pm 0.01$ at $150 \mathrm{GeV}$ and $0.92 \pm 0.01$ at $180 \mathrm{GeV}$. Using a truncated mean rather than the most probable value the corresponding numbers for the data are $0.99 \pm 0.02$ at $150 \mathrm{GeV}$ and at $180 \mathrm{GeV}$. Here the corresponding MC prediction is $0.95 \pm 0.02$ at $150 \mathrm{GeV}$ and $0.96 \pm 0.02$ at $180 \mathrm{GeV}$. The systematic error has been estimated using the maximum and minimum values obtained using different calibration runs or data at different impact points and varying the analysis 


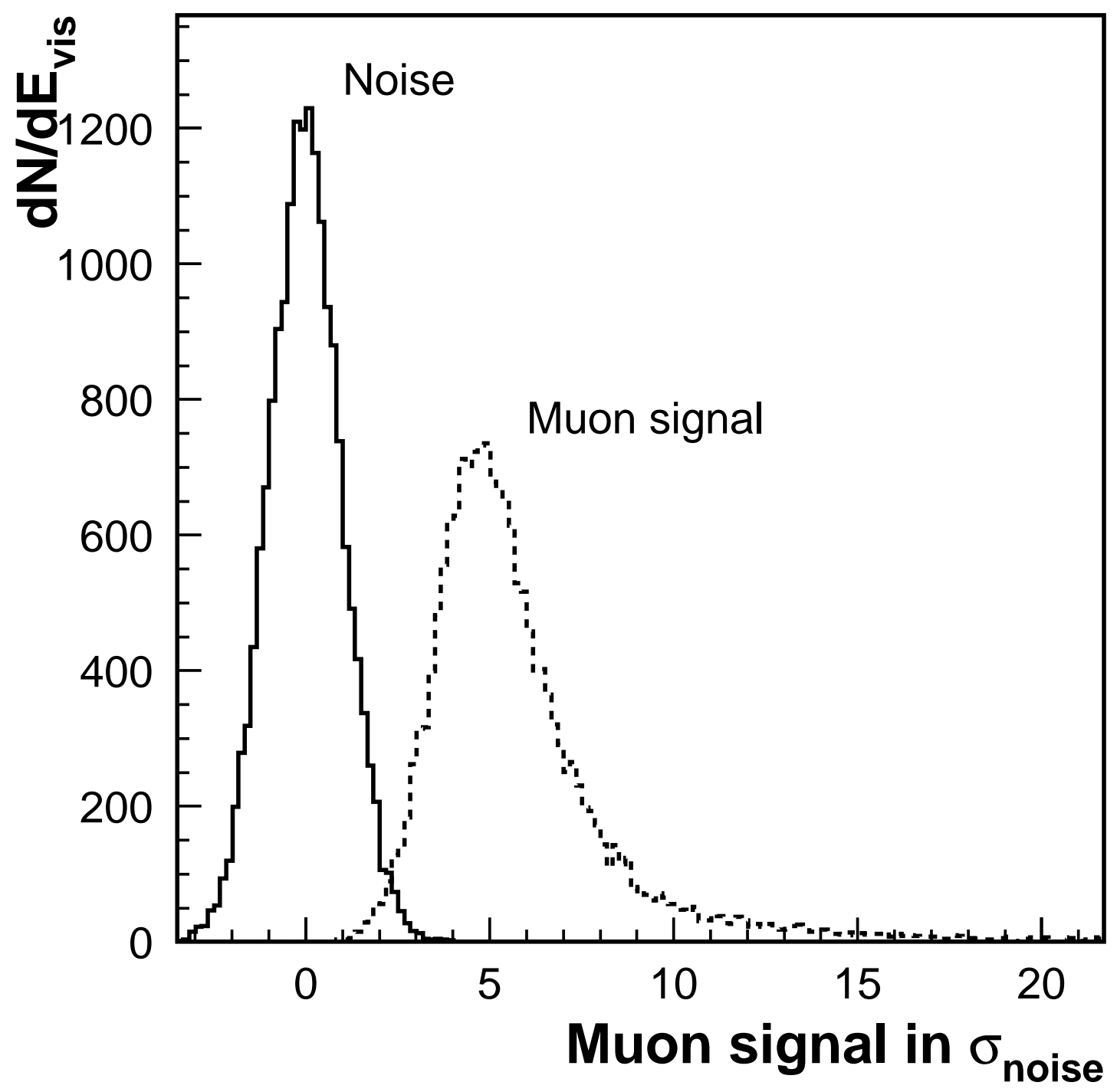

Figure 27: Response distribution for $180 \mathrm{GeV}$ muons (dashed line) in units of $\sigma_{\text {noise }}$, and the noise distribution (solid line). 


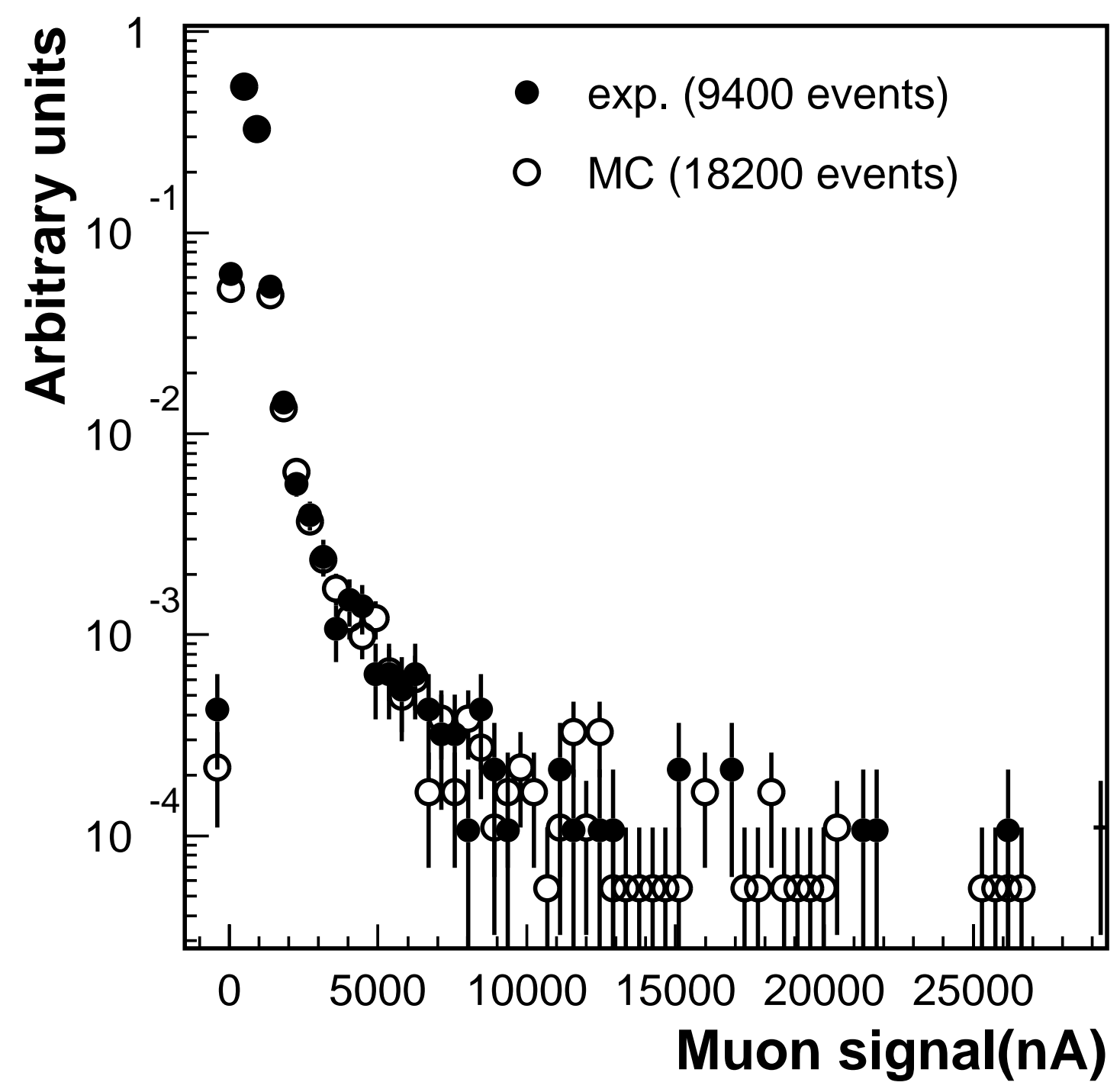

Figure 28: Response distribution for $120 \mathrm{GeV}$ muons, shown for beam test data (full circles) and simulation (open circles). 


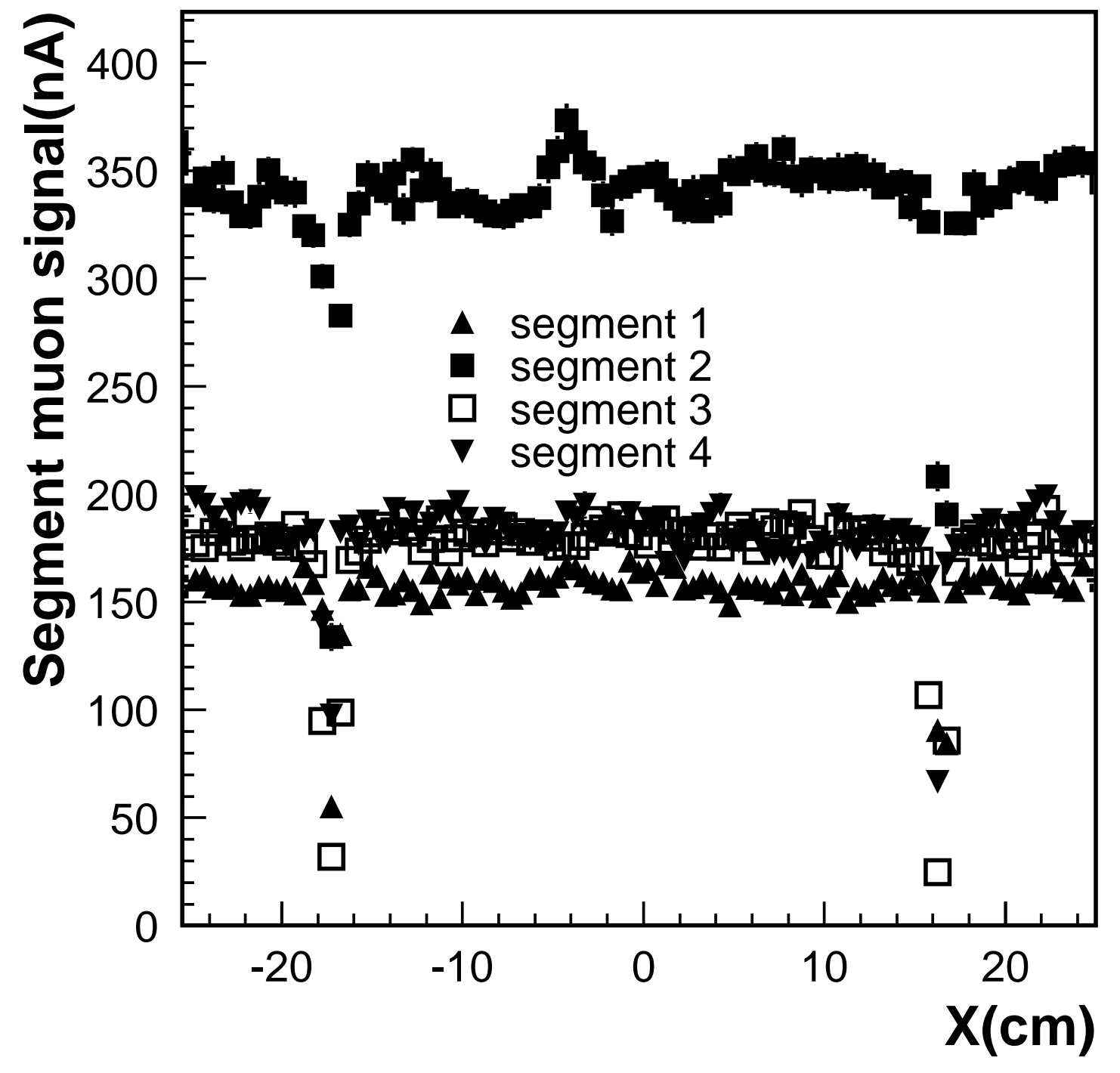

Figure 29: Horizontal scan with muons of $180 \mathrm{GeV}$ extending over almost the full $\phi$-range accessible. Shown is the reponse in each individual longitudinal segment. Clearly visible are the positions of the two crack zones between neighbouring modules. 

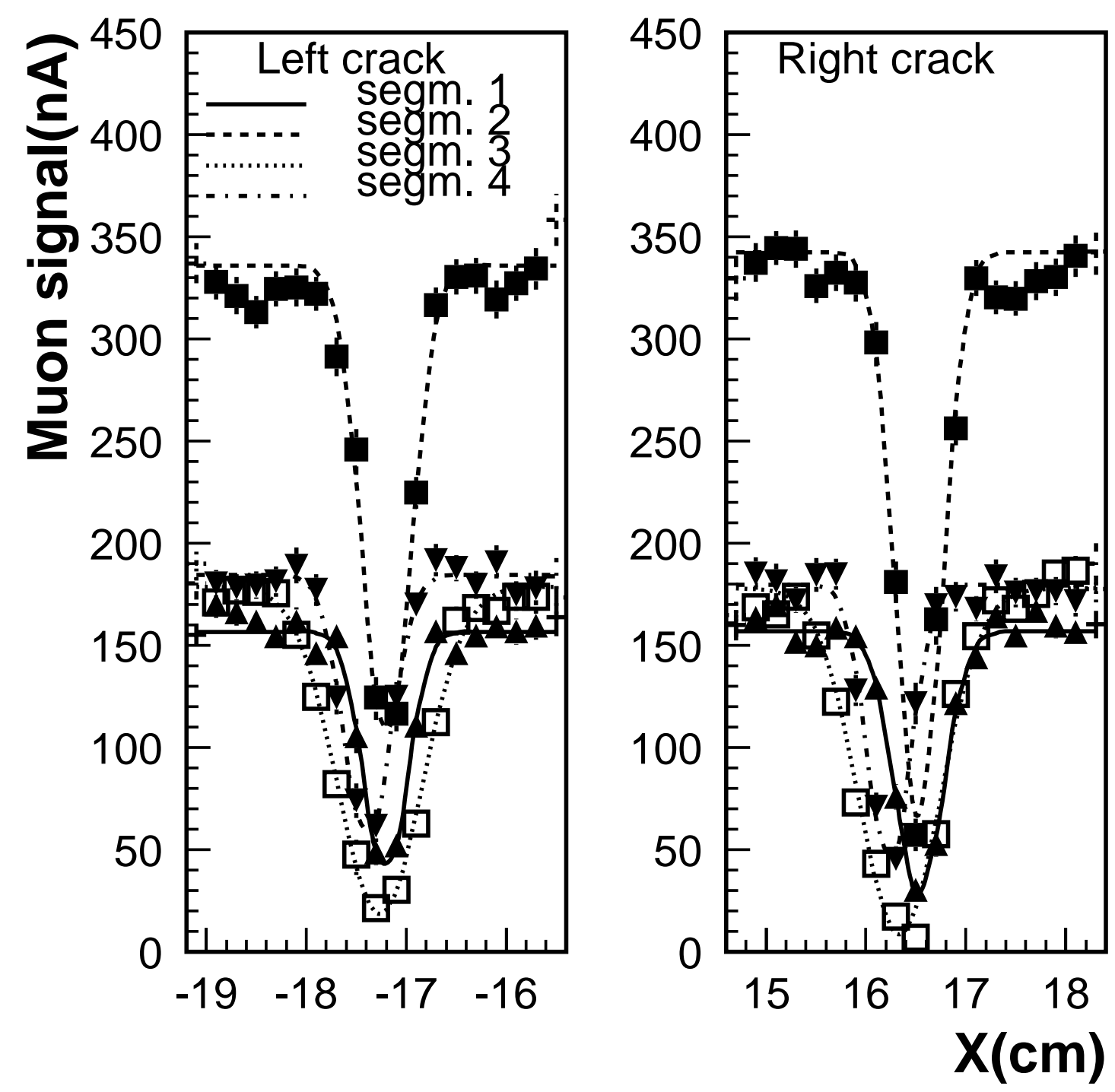

Figure 30: Horizontal scan with muons of $180 \mathrm{GeV}$. Shown is the signal in the two crack zones between neighbouring $\phi$-wedges in each individual longitudinal segment. 
cuts used. The electron to muon ratio can also be obtained using only the reponse in HEC1 or the total response in HEC1 and HEC2. Again, the maximum and minimum values obtained have been used to estimate the systematic error. From these studies we estimate the systematic error to be \pm 0.03 . The agreement between the data and the simulation is within errors.

Finally the data have been used to extract the electron to MIP (minimum ionizing particle) ratio. For this study the muon response at $150 \mathrm{GeV}$ in $\mathrm{HEC} 1$ has been used. The fractions of the ionization and electromagnetic energy deposit of muons in the energy range considered have been obtained from MC studies. For the electron to MIP ratio a value of $0.99 \pm 0.02$ has been obtained. This result has to be compared with the MC prediction of 0.94 . Taking the systematic error into account, the agreement is reasonable. 


\subsection{Response to Pions}

\section{Energy Reconstruction}

The total energy has been reconstructed using one hadronic calibration constant. Since the HEC is a non-compensating calorimeter, i.e. the $e / \pi$-ratio is energy dependent, this constant has been determined for each individual energy separately. To reconstruct the total energy, the signals in all read-out channels of the related cluster have been summed. The factor $\alpha_{\text {had }}$, transforming the visible energy $E_{n A}^{v i s}$ to the beam particle energy at a given nominal beam energy $E_{0}$, is obtained from the ratio

$$
\alpha_{\text {had }}\left(E_{0}\right)=E_{0} / E_{n A}^{\text {meas }} .
$$

Figure 31 shows for a given impact point the reconstructed pion signal at four different energies. To determine the width $\sigma$ and the maximum position $E^{\text {meas }}$ of the energy distribution, a gaussian fit (solid line) has been applied to the data. The fits have an acceptable $\chi^{2}$ but the distributions are slightly asymmetric due to enhanced low energy tails. Therefore the fit region has been limited to the interval $E^{\text {meas }} \pm 2 \sigma$ to reduce the influence of these tails on the result. The Figure 32 shows the energy dependence of the hadronic calibration constant $\alpha_{\text {had }}$ at four different impact points. It reflects directly the energy dependence of the $e / \pi$-ratio of the HEC.

\section{Energy Resolution}

Figure 33 shows the energy dependence of the energy resolution $\sigma / E$ for three different impact points. The noise as measured in the corresponding cluster, has been subtracted quadratically at each energy point. The resulting energy dependence may be parameterized using

$$
\sigma(E) / E=\frac{a}{\sqrt{E}} \oplus b .
$$

where $a$ represents the sampling term and $b$ the constant term. The lines indicate the results of the fits. For the data the fit yields typically $a=(70.6 \pm 1.5) \% \mathrm{GeV}^{1 / 2}$ and $b=(5.8 \pm 0.2) \%$. The data have been compared to the prediction from simulation, using GFLUKA, GCALOR and GHEISHA for the hadronic shower simulation. In general GCALOR yields the best description of the data, but still being not quite optimal. Figure 34 shows the energy dependence of the energy resolution for the impact point $\mathrm{J}$ and the corresponding prediction using GCALOR for the simulation of the hadronic shower. The lines show the result of the individual fits using the ansatz given above. GCALOR gives a somewhat too optimistic description of the energy resolution, reflected particularly in a smaller constant term: $b=(5.8 \pm 0.2) \%$ for the data and $b=(4.9 \pm 0.1) \%$ for the simulation.

To obtain the HEC intrinsic energy resolution the contribution due to energy leakage has to be unfolded. The increase of the energy resolution $\sigma$ due to leakage has been obtained from simulation for each energy and impact point [3]. Thus an intrinsic energy resolution has been derived. Typically we obtain for the sampling term $a=(62.2 \pm$ 1.8) $\% \mathrm{GeV}^{1 / 2}$ and for the constant term $b=(5.2 \pm 0.2) \%$. This can be compared with the corresponding GCALOR prediction of $a=(61.3 \pm 0.6) \% \mathrm{GeV}^{1 / 2}$ and $b=(3.5 \pm 0.1) \%$. 
The pion energy resolution can be improved using various weighting schemes. The corresponding improvement to the energy resolution has been found to be rather small. Typically only the constant term in the energy dependence of the energy resolution is reduced by about $0.6 \%$ in absolute size. To keep the procedure transparent and not to optimize for the energy resolution therefore only the simple approach using one overall calibration constant $\alpha_{\text {had }}$ has been used in the analysis presented.

\section{e/h Ratio}

An important intrinsic characteristic of a calorimeter is the sampling ratio for electrons $\left(e=E_{\text {vis }} / E_{\text {beam }} \equiv \alpha_{e m}^{-1}\right)$ and for hadrons $(\pi)$. For electrons this ratio does not depend on energy. This is not so for hadrons, where two components - an electromagnetic as well as hadronic part - contribute to the interaction: the real hadron response is a mixture of the electromagnetic component and the pure hadronic one. The energy dependence of these components governs the sampling ratio. Vice versa, one can extract from this energy dependence the intrinsic $e / h$ ratio ( $e$ and $h$ are energy independent values). The deviation of the $e / h$ ratio from 1 , typical for a non-compensating calorimeter, leads to a worsening of the energy resolution and gives rise to a constant term for the energy dependence of the energy resolution. The $e / h$ ratio and the measured $e / \pi$ ratio are related by the following Equation [33]

$$
e / \pi=\frac{e / h}{1+(e / h-1) \cdot f_{\pi^{0}}},
$$

where $f_{\pi^{0}}$ is the mean fraction of the initial pion energy deposited via electromagnetic cascades. $f_{\pi^{0}}$ is a slowly rising function with the energy and can be parameterized as [34]

$$
f_{\pi^{0}}=1-\left(\frac{E}{E_{0}^{\prime}}\right)^{m-1},
$$

For pions typical values are $E_{0}^{\prime} \approx 1 \mathrm{GeV}$ and $m \approx 0.85$. The parameter $m$ depends on very general properties of hadron-nucleus interactions and should not depend strongly on the type of calorimeter. Various fits of $m$ give values in the range 0.83 to 0.86 [34]. A slightly different ansatz [33] yields

$$
f_{\pi^{0}}=k \ln \left(\frac{E}{E_{0}^{\prime}}\right)
$$

with $k=0.11$.

The measured $e / \pi$ ratio is shown in Figure 35 (a) as well as the result of the fit using Equation 5 and Equation 6. These results are biased due to the energy leakage outside the chosen cluster and the given calorimeter acceptance. The correction factors obtained from simulation (GCALOR) depend only weakly on energy and amount to about $4.5 \%$ to $5.5 \%$. From a comparison of signals in read-out channels far from the impact point with simulation we estimate the error on this leakage correction to be $\pm 15 \%$, again based on the maximum variation seen.

The leakage corrected data are shown in Figure 35 (b). The resulting $e / h$ ratio obtained from the fit to the Equation 5 and Equation 6 with $m$ fixed to $0.85, e / h=$ 
$1.49 \pm 0.01$, is rather sensitive to the overall scale of the $e / \pi$ ratio: a change of 0.05 in $e / \pi$ leads to a change of 0.2 in $e / h$. The applied correction considerably improves the quality of the fit. The fit results for different impact points vary from $e / h=1.47 \pm 0.01$ to $e / h=1.52 \pm 0.01$ indicating that systematic errors are dominating over the statistical ones $( \pm 0.01)$. When using equation 7 [33] similar $e / h$ values are obtained, but with a substantially worse quality of the fit. From these studies we estimate the systematic error to be \pm 0.1 . This yields a value of $e / h=1.49 \pm 0.01 \pm 0.10$.

Simulation (GCALOR) results have been analyzed following the same procedure. The corresponding prediction is $e / h=1.32$.

\section{Longitudinal and Lateral Shower Profiles}

Figure 36 shows the energy dependence of the mean energy fraction (normalized to the total deposited energy) deposited in the individual longitudinal segment for the given pion cluster. Shown are the data (solid points) for the first (top left), second (top right), third (bottom left) and fourth (bottom right) longitudinal segment for a given impact point. The data reflect the energy dependence of the longitudinal shower profile. The energy fraction in the last segment is increasing noticeably with energy. Also shown are the various simulation (GCALOR) predictions (open points).

The granularity of the calorimeter is not fine enough to see details of the lateral shower shape. These details can be studied using the horizontal scan data obtained with pions at $200 \mathrm{GeV}$. The step size was $20 \mathrm{~mm}$. The signals in the vertical $(\eta)$ channels related to a given horizontal $(\phi)$ read-out channel have been summed to towers. Thus for the three $\phi$ wedges six $\phi$-towers can be studied for each longitudinal read-out segment. The comparison with simulation is shown in Figure 37. Shown is the signal in the $\phi$-tower five for the four longitudinal read-out segments. The center of this tower is at $-25.5 \mathrm{~cm}$. The increase of the hadronic shower size width with the longitudinal propagation of the hadronic shower is clearly visible. The simulation (GCALOR) gives a good description of the data, except for the extreme tails where GCALOR predicts a slightly larger shower size than observed in the data. Beam test data obtained with prototype modules have shown that GFLUKA and GHEISHA provide a good description of the data as well. 

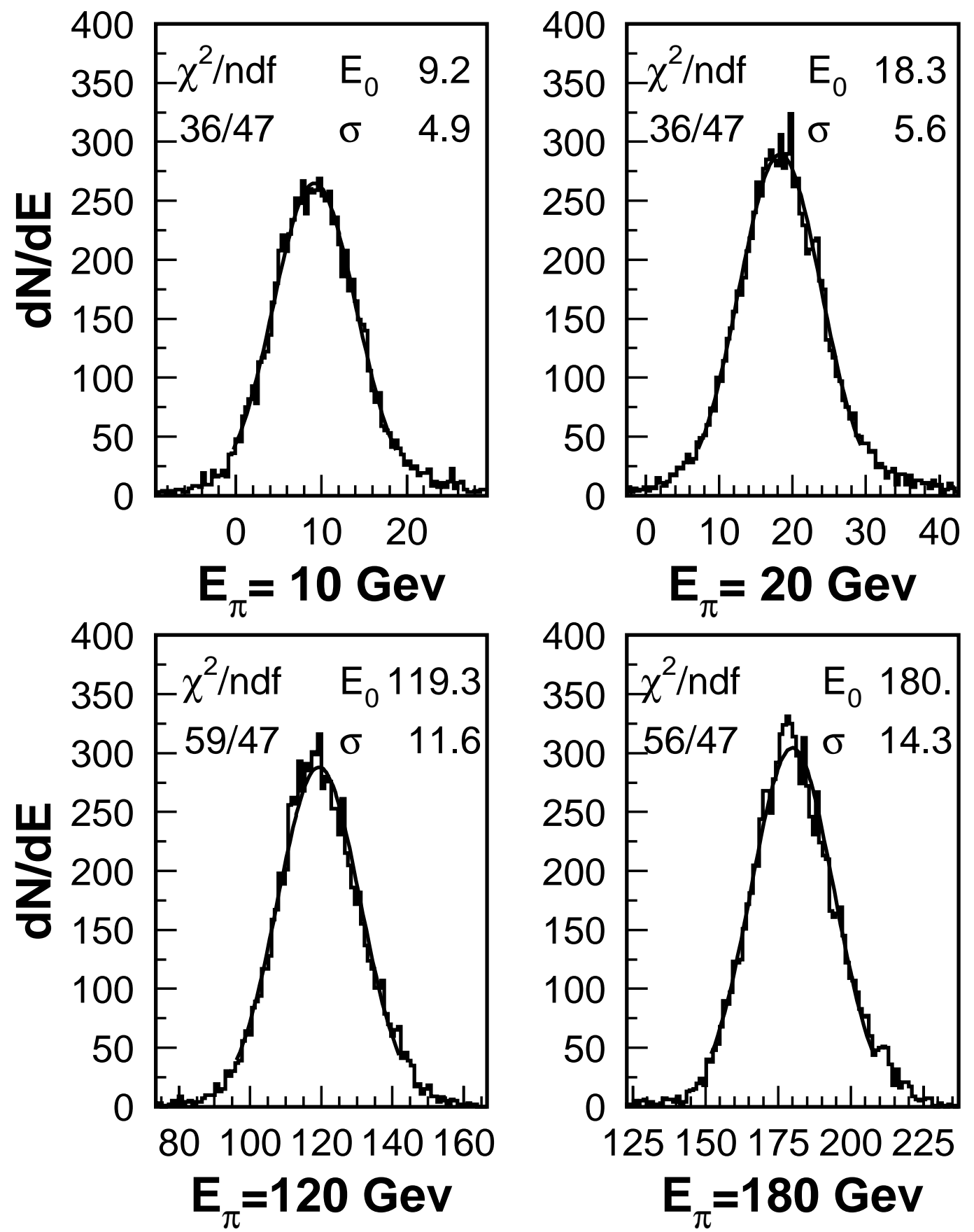

Figure 31: The reconstructed pion response at four different energies for a given impact point, showing the reconstructed energy $E_{0}(\mathrm{GeV})$, the width $\sigma(\mathrm{GeV})$ and the corresponding $\chi^{2}$ per number of degrees of freedom (ndf) of the fit (see text). 


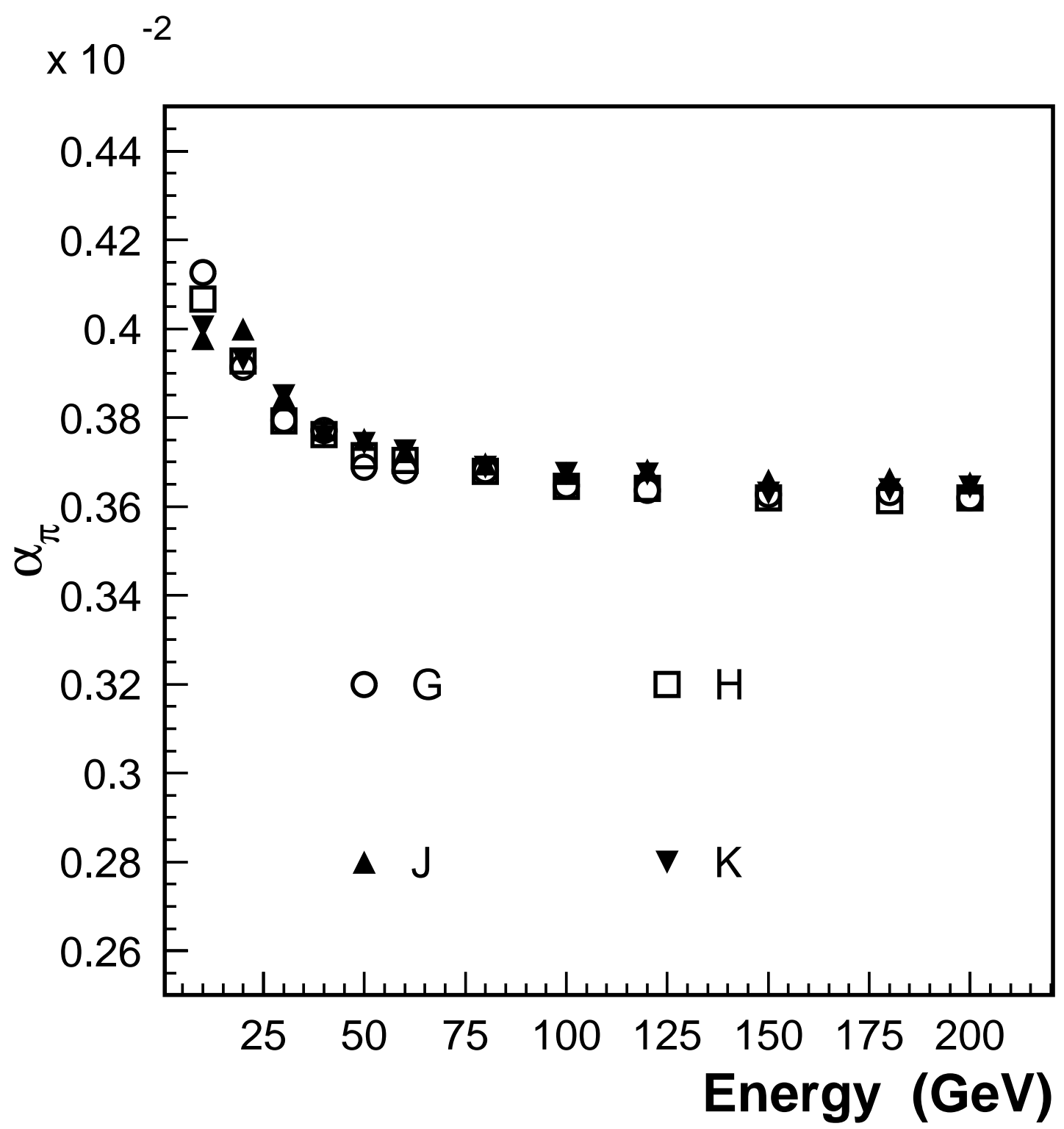

Figure 32: Energy dependence of the hadronic calibration constant at four different impact positions. 


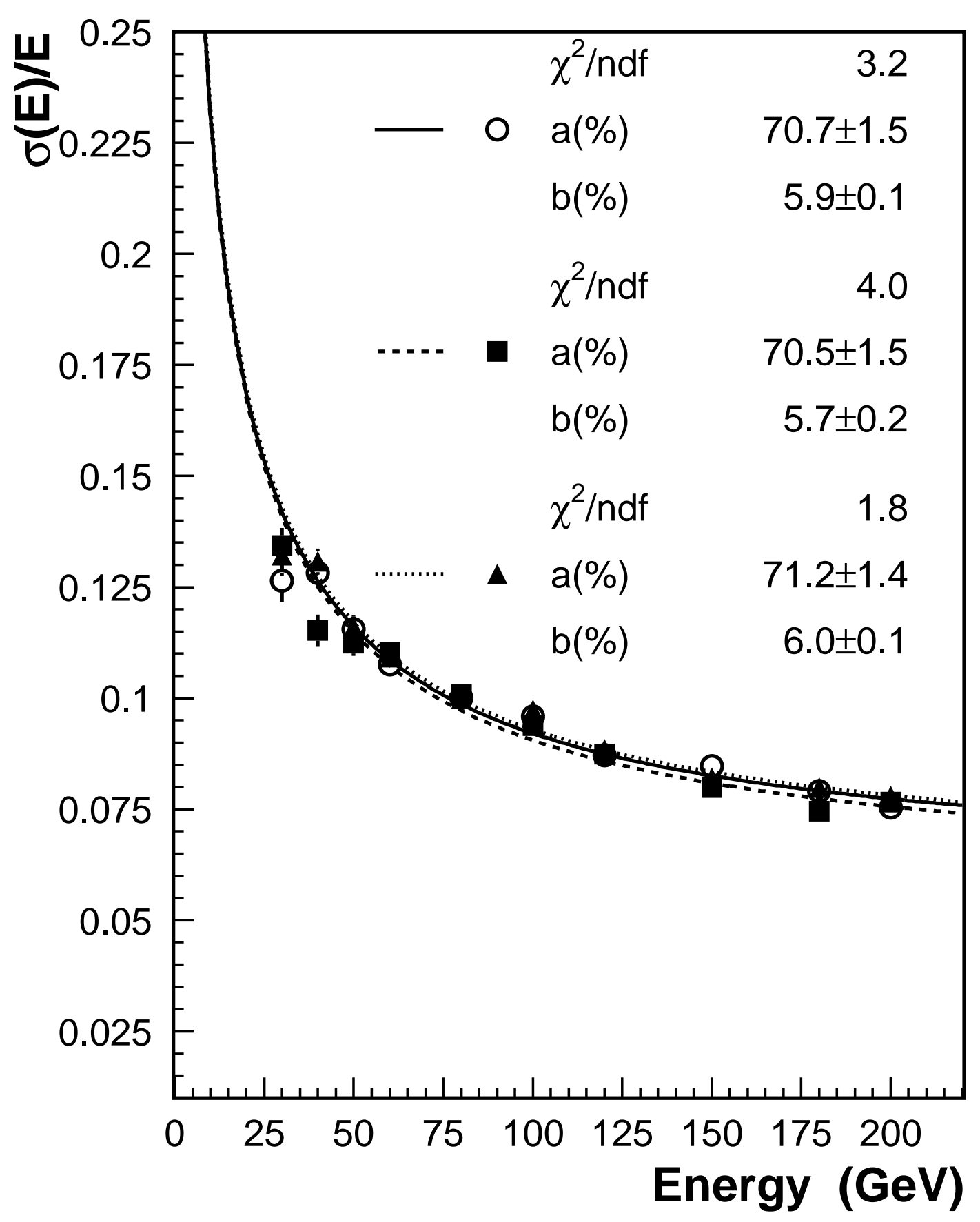

Figure 33: The energy dependence of the pion energy resolution. Shown are the data for three different impact points. The lines show the result of the individual fits (see text). 


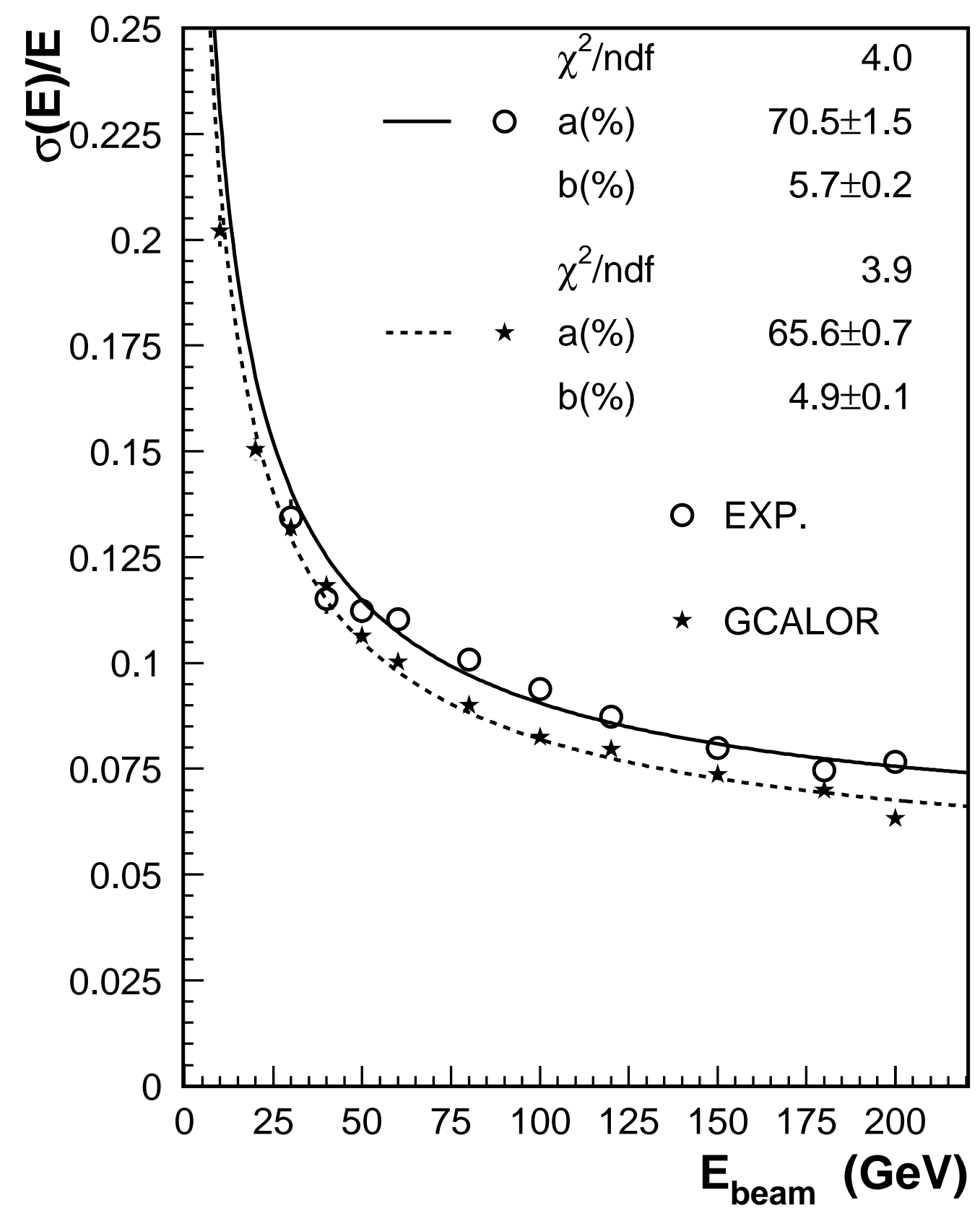

Figure 34: Comparison of the energy dependence of the pion energy resolution with the prediction from simulation. Shown are the data (full dots) for the impact point J as well as the corresponding prediction from simulation (stars) for the hadronic shower code GCALOR. The lines show the result of the individual fits (see text). 

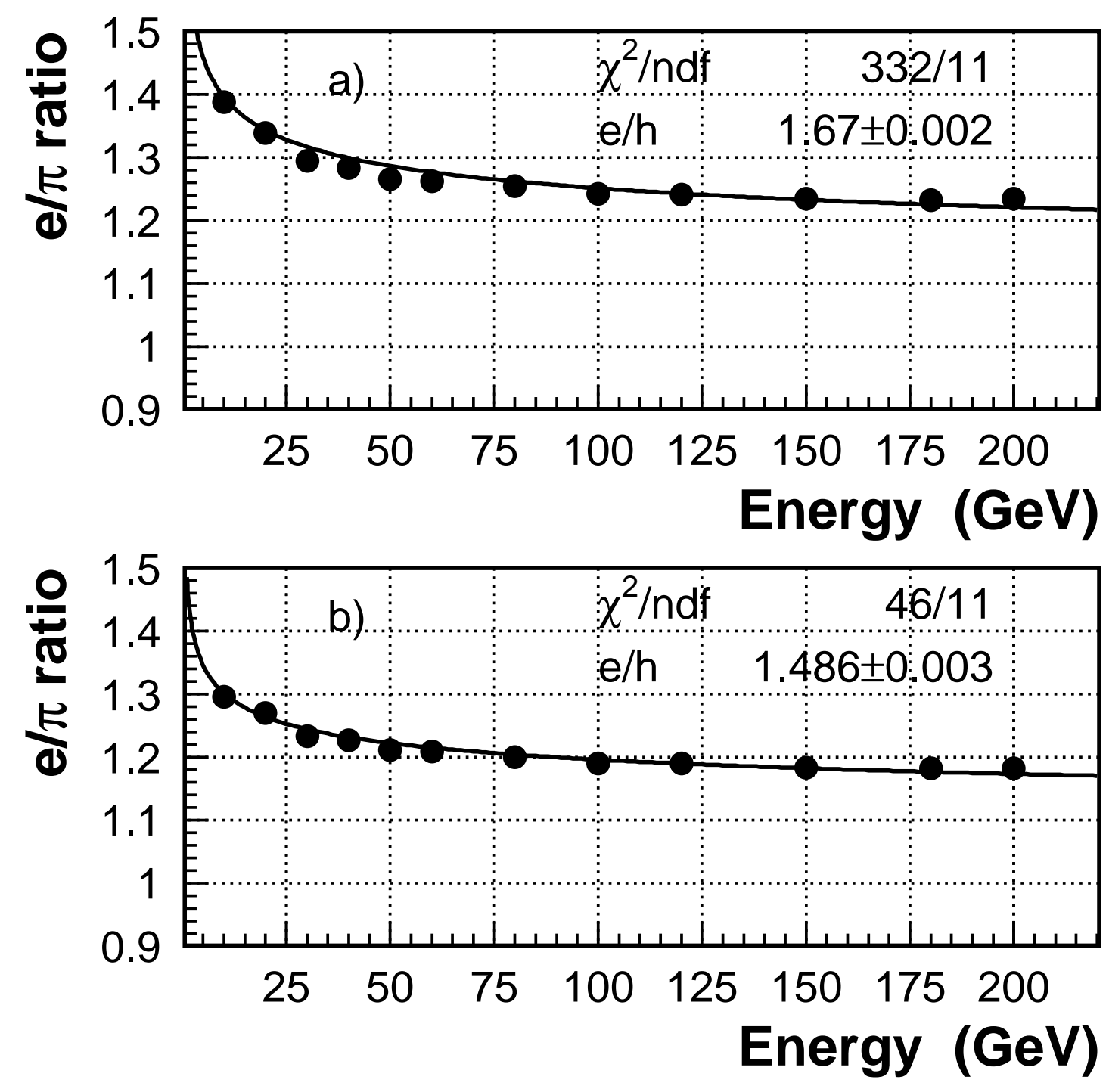

Figure 35: The energy dependence of the $e / \pi$-ratio for uncorrected data (a) and after correcting for energy leakage (b). The correction factor is rather energy independent. The solid line shows the result of the $e / h$ fit (see text). 

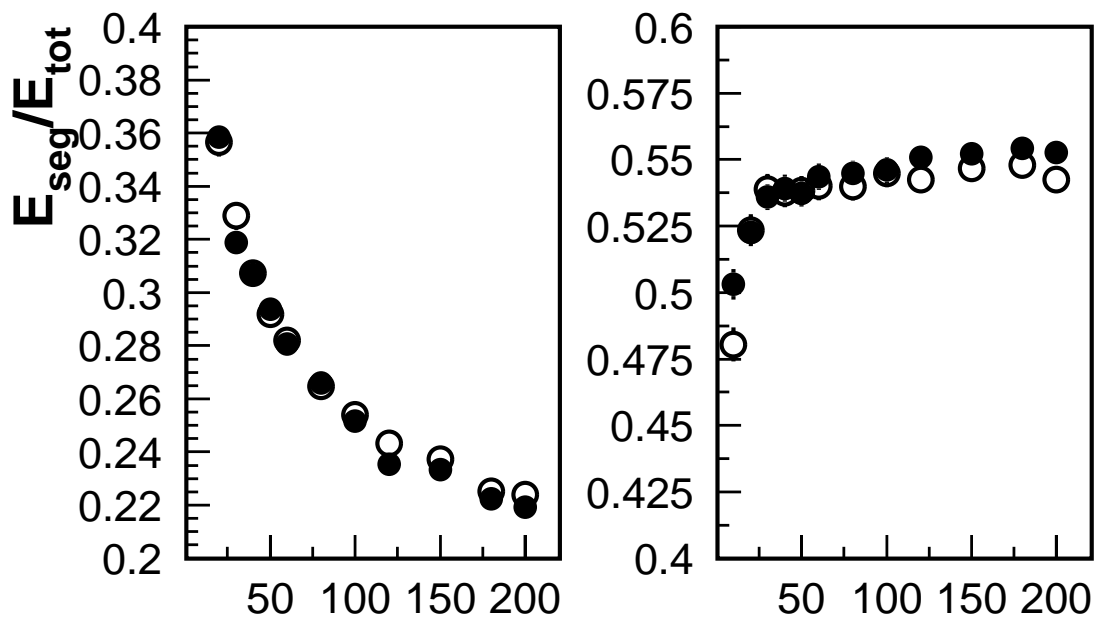

Energy
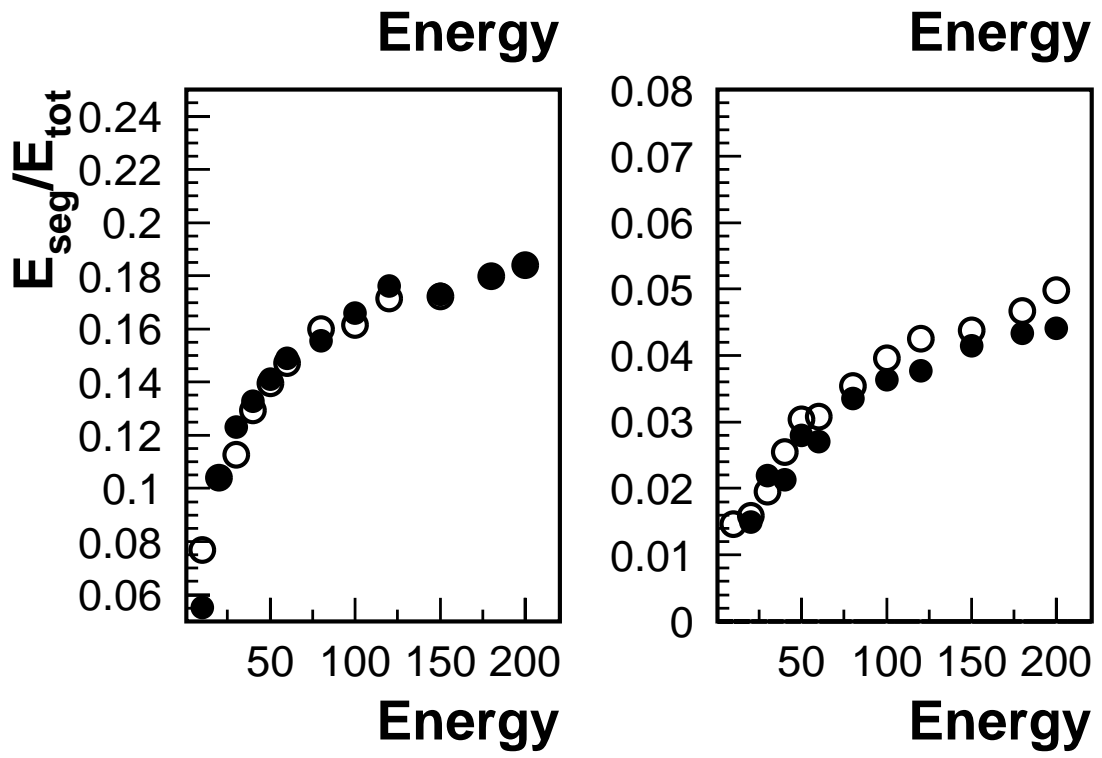

Figure 36: The energy dependence of the mean energy fraction deposited in the first (top left), second (top right), third (bottom left) and fourth (bottom right) longitudinal segment for a given impact point. Shown are the data (solid points) as well as the prediction from simulation (GCALOR, open points). 

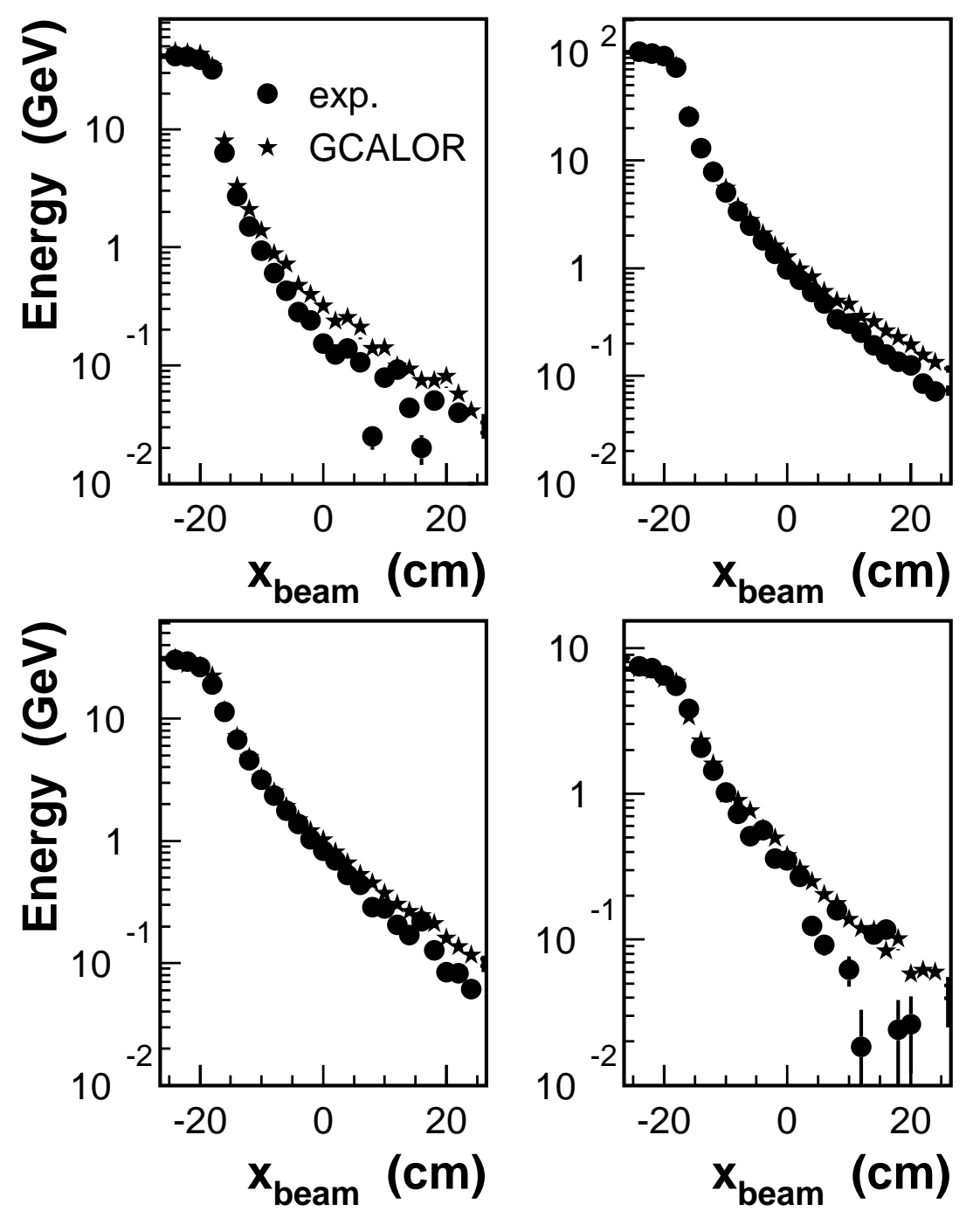

Figure 37: Lateral shower shape: Mean energy fraction in a $\phi$ tower ( $x$-tower in scan, center at $-25.5 \mathrm{~cm}$ ) in dependence on the $x$-position of the beam impact position. Shown are the data (solid points) for the four longitudinal read-out segments. For comparison the predictions from simulation (GCALOR, stars) are also shown. 


\subsection{Monte Carlo extrapolation to Jets in ATLAS}

In the ATLAS detector the hadronic end-cap calorimeter will be positioned behind the electromagnetic one. To evaluate the performance in the final ATLAS configuration detailed Monte Carlo studies using the simulation code GCALOR have been done [35].

Samples of single charged pions and jets in the energy range from 100 to $1000 \mathrm{GeV}$ have been fully simulated. The production angle has been chosen to be the centre of the end-cap region $(|\eta|=2.45)$. The energy reconstruction has been done at the so-called, hadronic-energy scale. The jet energy resolution has been determined by fitting Gaussian curves to the reconstructed energy distributions in an interval of $\pm 3 \sigma$ around the peak value. The mean value $E$ and the standard deviation $\sigma$ from this fit are used to calculate the resolution $\sigma / E$. The energy dependence of the resolution can be parameterised by the following ansatz:

$$
\frac{\sigma\left(E_{0}\right)}{E}=\frac{a}{\sqrt{E_{0}}} \oplus b
$$

where $E_{0}$ is the initial pion or jet energy, $a$ is the sampling term and $b$ is the constant term.

In Figure 38 the energy resolution obtained for single charged pions is plotted as function of initial energy. The fit with Equation (8) gives $a=(54 \pm 2) \% \mathrm{GeV}^{1 / 2}$ and $b=(2.6 \pm 0.1) \%$. The obtained values are better than expected from beam tests of the HEC modules because of the presence of the electromagnetic calorimeter in the front of the HEC, where the electromagnetic component of the jet is measured with an energy resolution of typically $10 \% / \sqrt{E(\mathrm{GeV})}$.

In Figure 38 the jet energy resolution is plotted as well together with the results of the fit with Equation (8). The jet energy resolution in the end-cap region is characterised by a sampling term $a=(56 \pm 3) \% \mathrm{GeV}^{1 / 2}$ and a constant term $b=(2.0 \pm 0.2) \%$.

These results characterise the intrinsic performance of the ATLAS calorimetry in the end-cap region: effects of noise and limited jet cone size for energy measurements have not been taken into account here.

\section{Conclusions}

Results of calibration runs with electrons, muons and pions have been presented.

For electrons an energy resolution of typically

$$
\frac{\sigma(E)}{E}=\frac{(21.4 \pm 0.2) \%}{\sqrt{E(\mathrm{GeV})}} \oplus(0.3 \pm 0.2) \%
$$

has been obtained. For muons a signal to noise ratio of about five has been determined. Using the most probable value the electron to muon ratio is $0.93 \pm 0.02 \pm 0.03(0.92 \pm$ $0.02 \pm 0.03)$ at $150 \mathrm{GeV}(180 \mathrm{GeV})$. From these results an electron to MIP ratio of $0.99 \pm 0.02 \pm 0.03$ has been extracted. These results compare well with expectations from simulation. 


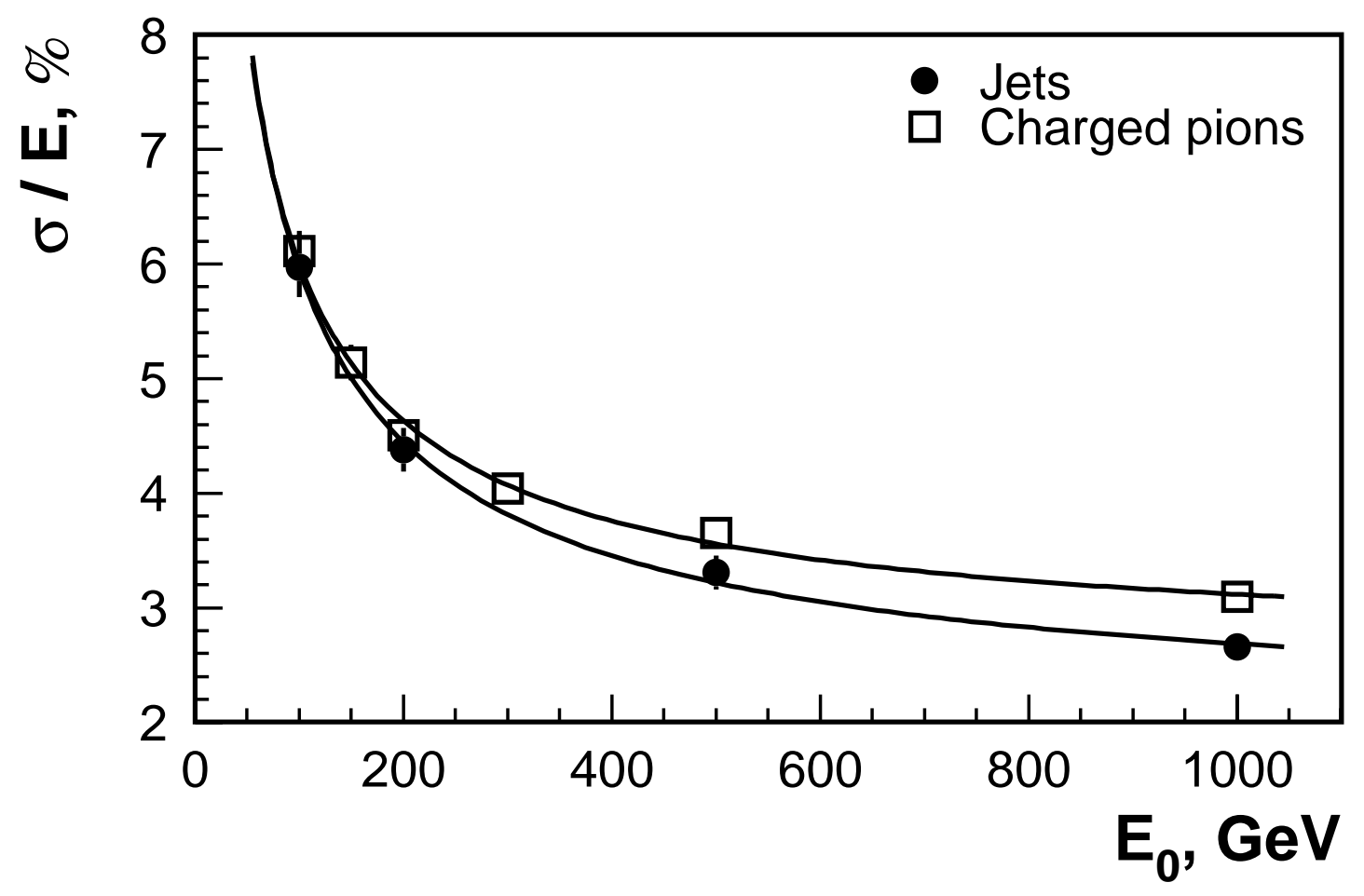

Figure 38: Energy dependence of the energy resolution for jets and single charged pions at $|\eta|=2.45$. The lines give fits with Equation (8).

For pions an energy resolution of

$$
\frac{\sigma(E)}{E}=\frac{(70.6 \pm 1.5) \%}{\sqrt{E(\mathrm{GeV})}} \oplus(5.8 \pm 0.2) \%
$$

has been obtained. The intrinsic electron to hadron ratio is $1.49 \pm 0.01 \pm 0.10$.

\section{Acknowledgements}

The support of the CERN staff operating the SPS and the H6 beam line, in particular the continuous help of K. Elsener, is gratefully acknowledged. We thank D. Sauvage and the ATLAS-LAr cryogenics operations team for their invaluable help.

This work has been supported by the Bundesministerium für Bildung, Wissenschaft, Forschung und Technologie, Germany, under contract numbers $05 H A 8 E X 16$, $05 H A 8 U M A 8$ and $05 H A 8 P X A 2$, by the Natural Science and Engineering Research Council of Canada and by the Slovak funding agency VEGA under contract number $2 / 5138$. We thank all funding agencies for financial support. 


\section{References}

[1] The ATLAS Collaboration, ATLAS Liquid Argon Calorimeter Technical Design Report, CERN/LHCC/96-41, ATLAS TDR 2, 15 December 1996.

[2] The ATLAS Collaboration, ATLAS Calorimeter Performance Design Report, CERN/LHCC/96-40, ATLAS TDR 1, 13 January 1997.

[3] A.A. Minaenko, Analysis of Testbeam Data, obtained with Module Zero of the Hadronic End-cap Calorimeter, ATLAS Internal Note LARG-99-011, April 99.

[4] A. Cheplakov et al., Energy Resolution of the HEC Prototype from the Pion 1996 Data, ATLAS Internal Note LARG-98-097, 1998.

[5] I. Grubmeier et al., Analysis of Electron and Pion Testbeam Data of the Hadronic End-cap Calorimeter, ATLAS Internal Note LARG-98-096, 1998.

[6] M. Dobbs et al., Hadronic End-cap Modules Zero: Pion and Electron Energy Scan Analysis from April 1998 Testbeam Data, ATLAS Internal Note LARG-99-001, 1999.

[7] D. Fortin and M. Lefebvre, Performance of the ATLAS Hadronic End-cap Calorimeter Modules to Electrons and Pions from 1999 Beam Test Data, ATLAS Internal Note LARG-2001-010, 2001.

[8] J. Colas, M.Pripstein and W.A. Wenzel, Nucl. Instr. and Meth. A294 (1990) 583.

[9] C. Leroy et al., Liquid Argon Pollution Tests of the ATLAS Detector Materials at IBR-2 Reactor in Dubna, Particle and Nuclei, Letters 5 (2000), 5.

[10] J. Ban et al., Design and Performance of the cold GaAs electronics of the ATLAS Hadronic End-Cap Calorimeter, to be published in Nucl. Instr. and Meth.

[11] J. Ban et al., Cold Electronics of the ATLAS Hadronic End-Cap Calorimeter, to be published in Nucl. Instr. and Meth.

[12] M. Chalifour, User and Maintenance Guide for Testbeam Cryostats, Internal Note 6N 5710Q-321500PA, Saclay, 1999.

[13] M. Lefebvre and D. O’Neil, ATLAS internal note ATL-LARG-99-002, January 1999.

[14] J. Collot et al., The LAr tri-gain shaper, ATLAS Internal Note LARG-No-92, March 1998.

[15] VIC 8251F, VIC user's manual, VIC to VME Interface with Mirrored Memory, CES, 1995.

[16] RAID 8235 VME RISC Processor Board User's Manual, Creative Electronic Systems, Geneva, 1995.

[17] J. Colas et al., The LArg calorimeter calibration board, ATLAS Internal Note LARG2000-006, December 1999. 
[18] H. Brettel et al., Calibration of the ATLAS Hadronic End-Cap Calorimeter, Proceedings of the Sixth Workshop on Electronics for LHC Experiments, Krakow, Poland, September 11-15, 2000, CERN/LHCC/2000-041.

[19] R. Bernier et al., SPAC: Serial Protocol for the Atlas Calorimeter, ATLAS Internal Note LARG-98-093, March 1998.

[20] R. Chytracek, CALTCP 1.0 Unix Network Programmer's Library, http://chytrace.home.cern.ch/chytrace/caltcp/

[21] R. Brun and F. Rademakers, ROOT - An Object Oriented Data Analysis Framework, Nucl. Instr. and Meth. A389 (1997) 81, http://root.cern.ch/

[22] W.E. Cleland and E.G. Stern, Signal processing consideration for liquid ionization calorimeters in a high rate environment, Nucl. Instr. and Meth. A338 (1994) 467.

L. Kurchaninov and M. Levitsky, Optimal Weighting of Signal Samples for LAr Calorimeters, ATLAS internal note LARG-NO-044, 14 July 1996.

[23] W.E. Cleland et al., Dynamic range compression in a liquid-argon calorimeter, VI International Conference on Calorimetry in High Energy Physics, ICCHEP '96, Frascati, Italy, Frascati physics series, 6 (1996) 849.

[24] W. Walkowiak, PhD-thesis, Entwicklung von Flüssig-Argon-Reinheitsmeßgeräten für das ATLAS-Experiment und Messungen zur Energieauflösung eines hadronischen Flüssig-Argon-Kalorimeters, Shaker Verlag, Aachen 1999.

[25] The cold preamplifiers for the purity monitoring system were developed and kindly provided by V. Radeka (Brookhaven National Laboratory, NY, USA).

[26] V. Kukhtin et al., The ATLAS-LARG Temperature Measuring System, to be published in Nucl. Instr. and Meth.

[27] A. Kiryunin and D. Salihagic, Monte Carlo for the HEC Prototype: Software and Examples of Analysis, HEC Note-063 (1998).

[28] A. Artamonov et al., DICE-95, ATL-SOFT-95-014 (1995).

[29] R. Brun et al., GEANT 3, CERN DD/EE/84-1, 1986.

[30] W. Walkowiak, Drift velocity of free electrons in liquid argon, Nucl. Instr. and Meth. A449 (2000) 288.

[31] J. Thomas and D.A. Imel, Recombination of electron-ion pairs in liquid argon and liquid xenon, Phys. Rev. A36 N.2 (1987) 614.

[32] R.T. Scalettar et al., Critical test of geminate recombination in liquid argon, Phys. Rev. A25 N.4 (1982) 2419.

[33] R. Wigmans, Nucl. Instr. and Meth. A259 (1988) 273. 
[34] D. Groom, What really goes on in a hadronic calorimeter, VII International Conference on Calorimetry in High Energy Physics, Tuscon, Arizona, November 9-14, 1997.

[35] S. Chekulaev et al., Performance of the ATLAS Hadronic End-Cap Calorimeter, Proceedings of the Eighth International Conference on Calorimetry in High Energy Physics, Lisbon, Portugal, (1999) 721. 